\title{
Hydrogen Sensing with Long-Range Surface Plasmon Membrane Waveguides
}

\author{
by \\ Norman R. Fong, B. Eng., M.A.Sc.
}

A thesis submitted to the Faculty of Graduate and Postdoctoral Affairs in partial fulfillment of the requirements for the degree of

\section{Doctor of Philosophy}

in

Electrical Engineering

Ottawa-Carleton Institute for Electrical and Computer Engineering

Carleton University

Ottawa, Ontario

(C) 2015, Norman R. Fong 


\section{Abstract}

This integrated article thesis presents the full design, fabrication, characterization and testing of a long-range surface plasmon polariton (LRSPP) cladded membrane waveguide hydrogen gas $\left(\mathrm{H}_{2}\right)$ sensor with integrated grating couplers.

The sensor, which is the first of its kind to be demonstrated in the literature, features a thin gold $(\mathrm{Au})$ stripe embedded in an ultra-thin free standing Cytop membrane with palladium (Pd) transducer. The design is performed through finite element method (FEM) optical modeling of the LRSPP waveguide and gratings. The non-trivial fabrication process utilizes facilities at Carleton University and the University of Ottawa and is presented in detail. The process involves multi-layer dielectric deposition, blind double-sided wafer alignment, multiple metal depositions using photolithography and ebeam lithography as well as a through-wafer silicon wet etch step.

The devices are passively characterized using an optical cutback technique comparing the observed waveguide attenuation to that of simulated values showing good agreement. Sensing tests are performed with hydrogen concentrations up to $3 \%$. A best detection limit of $290 \mathrm{ppm}$ is observed with a response time of $7 \mathrm{~s}$ to $0.6 \% \mathrm{H}_{2}(99.4 \%$ $\mathrm{N}_{2}$ ). The sensor has the capability of a higher dynamic range than other thin film sensors while other simple adjustments are discussed that can be applied to improve overall performance. 


\section{Acknowledgements}

Foremost I would like to thank my supervisors Dr. R. Niall Tait and Dr. Pierre Berini for their expertise, support and encouragement over these many years. I would like to acknowledge the Carleton University Microfabrication staff: Rob Vandusen, Angela McCormick, Rick Adams and Carol Adams as well as the lab managers from the Berini Group at the University of Ottawa: Ewa Lisicka-Skrzek and Anthony Olivieri. I have always appreciated your patience and willingness to help without reservation. Sincere thanks also to Mr. Nagui Mikhail from the Carleton University Department of Electronics for his help with countless little things that allowed me to complete this one large thing. I have learned many skills from working as a teaching assistant under several professors; it was always a pleasure Dr. John Rogers, Dr. Winnie Ye, Dr. Calvin Plett, Dr. Jacques Albert, Dr. N. Garry Tarr and Dr. Q.J. Zhang.

Finally, I would also like to acknowledge the other students and researchers who struggled alongside me in the labs over the years: Richard Daviau, Charles Chiu, Raza Hanif, Hamoudi Asiri, Sa'ad M. Hassan, Lloyd Duma, Ehsan Ghias-Begloo, Dr. Jeremy Upham, Behnam Banan, Martin Dussault, Howard Northfield and Wei Ru Wong. 


\section{Table of Contents}

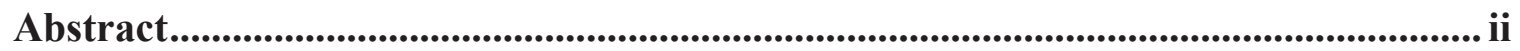

Acknowledgements ............................................................................................................................. iii

Table of Contents .......................................................................................................... iv

List of Illustrations.......................................................................................................................... vi

1 Chapter: Introduction ............................................................................................. 1

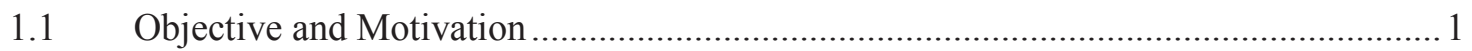

1.2 Thesis outline

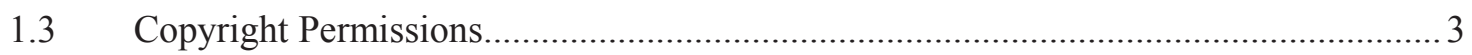

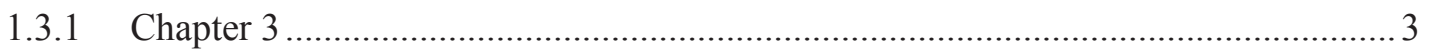

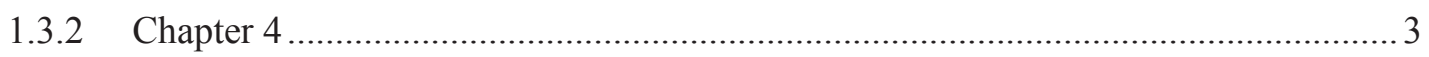

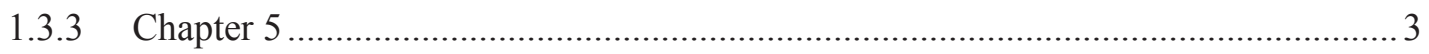

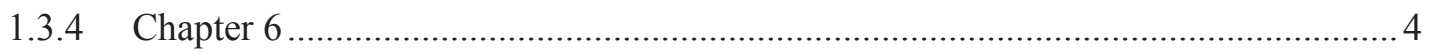

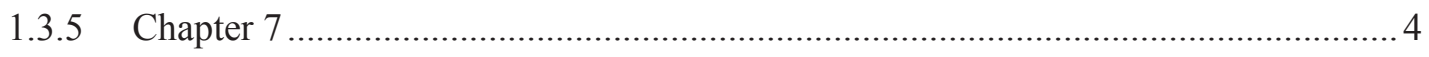

2 Chapter: Background \& Literature Review ................................................................. 5

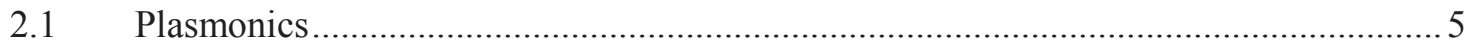

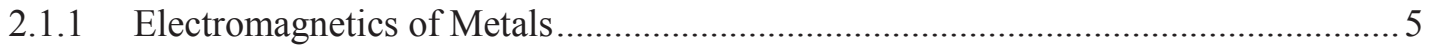

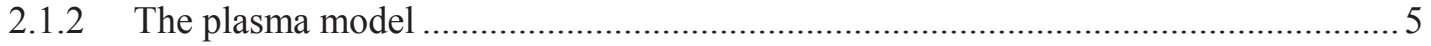

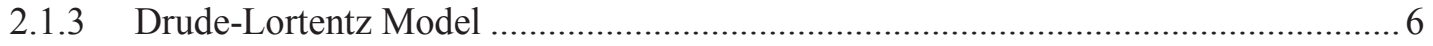

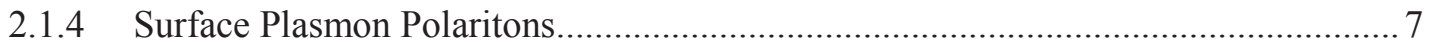

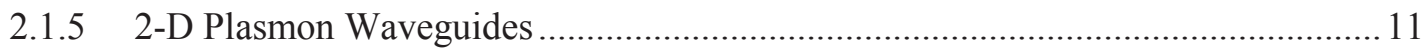

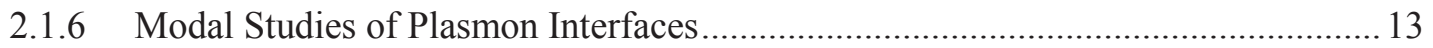

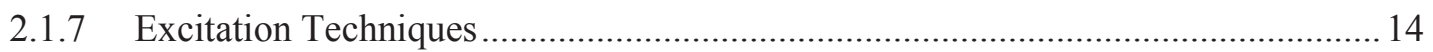

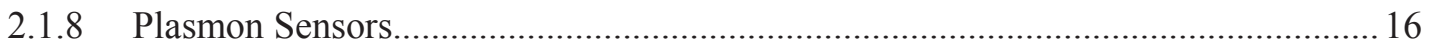




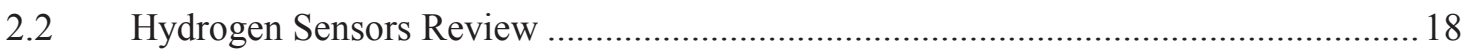

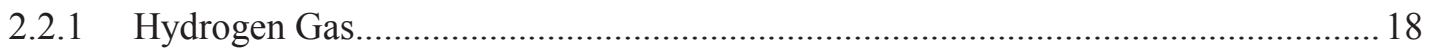

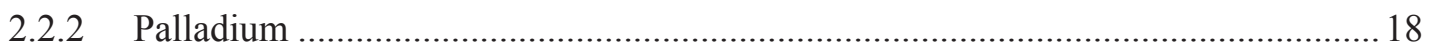

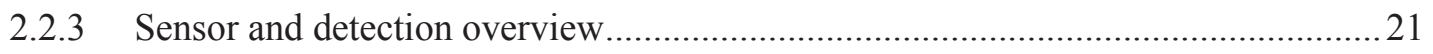

2.2.4 Plasmonic Hydrogen Sensors Review................................................................23

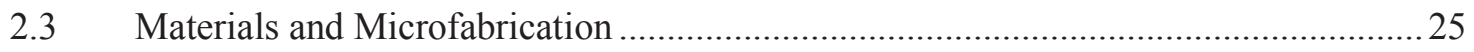

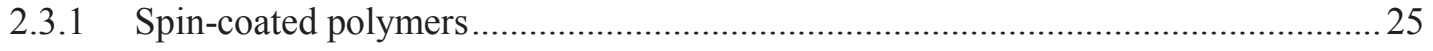

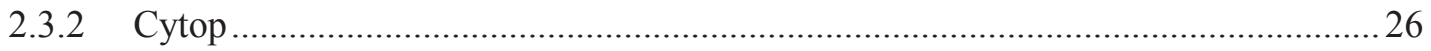

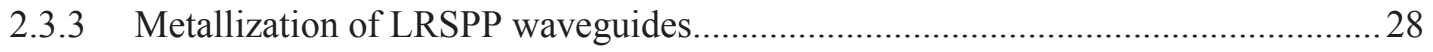

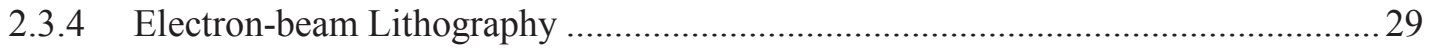

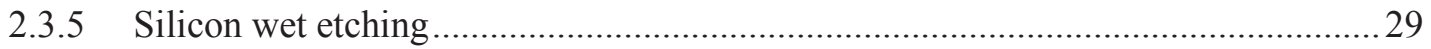

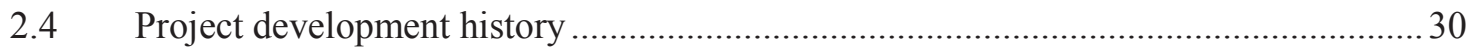

3 Chapter: Modeling of Plasmonic Hydrogen Gas Sensors .................................... 35

4 Chapter: Design of the LRSPP Membrane Waveguide Sensor........................... 43

5 Chapter: Fabrication Methods ..................................................................................... 53

6 Chapter: Optical Characterization of LRSPP Waveguides................................ 61

7 Chapter: Hydrogen Sensing.......................................................................... 72

8 Chapter: Conclusions and Future Work ................................................................. 79

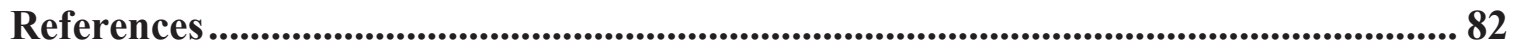




\section{List of Illustrations}

Figure 1 Diagram of coupled excitation between an EM wave (curved lines) and electron

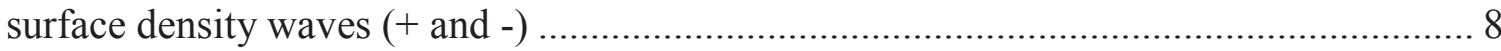

Figure 2 1-D electric field profiles for (a) Single-interface SPP (b) 2-interface SPPs with large metal thickness (c) coupled SPP modes with thin metal layer 9

Figure 3 (a) Metal slab waveguide cross-section (infinite in $\mathrm{x}$, propagation in $\mathrm{z}$ ) (b) Metal stripe of finite width. The substrate and cladding are not necessarily the same, but $\varepsilon_{d l}=\varepsilon_{d 2}$ is required for LRSPP propagation

Figure 4 Cross sections for (a) LRSPP membrane waveguide (b) Cladded membrane waveguide

Figure 5 Dispersion relation for a single interface SPP ………………........................ 15

Figure 6 Example of a grating coupled SPP shown as a step-in-height of the metal ....... 16 Figure 7 Diagram of hydrogen atom distribution with in the Pd lattice for the various hydride phases. Reprinted (adapted) with permission from [26]. Copyright 2014 American Chemical Society.

Figure 8 (a) Pressure-composition-isotherms for Pd and (b) magnified view of the operating range of interest. Modified with permission from [28]. Copyright 2007 IOP Institute of Physics.

Figure 9 Various SPR hydrogen sensors (a) Kreschmann geometry [46] (b) LRSPP Kreschmann with 1D PC [47] (c)-(d) Pd coated fibers [48,49] (e) Pd coated channel waveguide [50]......

Figure 10 Molecular formula for Cytop polymer ............................................................ 26

Figure 11: Overview of bi-layer lift off metallization ................................................... 28 
Figure 12 LRSPP waveguide history (a) original membrane waveguide on nitride [20] (b) membrane waveguide on Cytop [58] (c) Uncladded LRSPP biosensor waveguide [59]. (d) LRSPP biosensor waveguide with microfluidic channels [60] (e) First generation membrane waveguide hydrogen sensor - $\mathrm{CH} 4$ (f) Second generation (g) Cladded

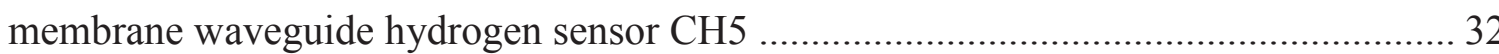

Figure 13 (a) Electric field (y-component) distribution over the cladded membrane waveguide hydrogen sensor cross section (b) Electric field distribution through the structure center showing maximum electric fields along the $\mathrm{Pd} /$ air interface 


\section{Chapter: Introduction}

\subsection{Objective and Motivation}

Plasmonics is the study of quantized plasma oscillations in metals due to electromagnetic interactions. This effect was first observed in 1902 by Wood [1] as an unexplained optical reflection in a metal grating. These effects were further studied and ultimately explained as the collective oscillations of free electrons coupled to optical waves $[2,3]$ with the first theoretical description of surface plasmons in 1957 by Ritchie [4]. The field has since seen massive growth as surface plasmons provide an extremely enticing potential for nanoscale optical confinement which can have intriguing applications as will be discussed in Chapter 2. This growth has been spurred by recent advancements in micro- and nano-fabrication along with increasing computational power allowing for the creation of increasingly complex structures.

The most common application field for plasmonic devices is sensors. The properties of plasmons allow for extremely sensitive sensors that can potentially supplant existing technologies, specifically in the fields of biosensing and gas sensing. However, few commercial plasmonic based sensors currently exist. The main drawback is that the devices end up having large losses (high optical attenuation) which limits their applications. Another drawback is that many common implementations require either bulky or inconvenient apparatus to use the sensors or to couple signals.

Hydrogen gas $\left(\mathrm{H}_{2}\right)$ is reactive and easily combustible in air, and thus poses a significant safety concern. The hydrogen economy is rapidly growing with the commercialization and miniaturization of fuel cells for clean energy production in addition to incumbent industrial chemical and processing applications. The National 
Renewable Energy Laboratory (NREL) and U.S. Department of Energy (DOE) have established an ideal set of standards [5] for hydrogen sensors (discussed in Section 2.2.3).To date, no sensor has been demonstrated to meet all of these strict requirements.

This thesis presents a hydrogen sensor based on the long-range surface plasmon polariton (LRSPP) cladded membrane waveguide architecture which attempts to address these sensing requirements.

\subsection{Thesis outline}

The dissertation material is presented in an integrated article format with each chapter being an as-published or in-review scientific journal article with self-contained background/theory, discussion, references and page numbering. As a result some of the material may be repeated among the articles, particularly introductory matter. The references for non-article materials can be found in the References section at the end of this document.

Chapter 2 presents background material and a literature review on plasmonics, hydrogen sensors and microfabrication. Chapter 3 begins the presentation of the thesis content with the design of the first generation hydrogen sensor. Although this structure was not ultimately used, it provides a foundation for the final device. Chapter 4 presents the design details of the final structure. The fabrication methods are described in Chapter 5. Chapters 6 and 7 present the optical characterization and sensing results respectively. 


\subsection{Copyright Permissions}

\subsubsection{Chapter 3}

The article in this chapter is presented in full and is reprinted with the permission of Elsevier Limited.

[CH3] N. R. Fong, P. Berini and R. N. Tait, "Modeling and design of hydrogen gas sensors based on a membrane-supported surface plasmon waveguide", Sensors and Actuators B: Chemical 161 (1), 2012. http://dx.doi.org/10.1016/j.snb.2011.10.033

\subsubsection{Chapter 4}

The article in this chapter is presented in full and is reprinted with the permission of AIP Publishing LLC.

[CH4] N. R. Fong, P. Berini and N. Tait, "Modeling of long range surface plasmon polariton cladded membrane waveguides with integrated grating couplers as hydrogen sensors", Journal of Applied Physics 117, 2015, http://dx.doi.org/10.1063/1.4919758

\subsubsection{Chapter 5}

The article in this chapter is presented in full and is reprinted with the permission of AIP Publishing LLC. 
[CH5] N. R. Fong, P. Berini and N. Tait, "Fabrication of long-range surface plasmon hydrogen sensors on Cytop membranes integrating grating couplers", Journal of Vacuum Science and Technology B 33, 2015, http://dx.doi.org/10.1116/1.4906827

\subsubsection{Chapter 6}

The article in this chapter is presented in full and is reprinted with the permission The Optical Society (OSA).

[CH6] N. R. Fong, P. Berini and N. Tait, "Characterization of grating-coupled long range surface plasmon polariton membrane waveguides", Optics Express 23, 2015, http://dx.doi.org/10.1364/OE.23.017421

\subsubsection{Chapter 7}

As of the publication of this thesis, this article has been submitted to the Royal Society of Chemistry journal Nanoscale and is currently under review. 


\section{Chapter: Background \& Literature Review}

\subsection{Plasmonics}

\subsubsection{Electromagnetics of Metals}

The interactions of electromagnetic (EM) waves with metals at frequencies up to the visible spectrum are described by classical theory based on Maxwell's equations.

Traditionally, metals were used in circuitry and guiding of EM waves in the microwave or far-infra red spectral regions. In these applications metals can be considered perfect electric conductors (PEC) in which the restoring force of the conduction electrons cancel the field from an external charge, effectively preventing propagation of waves through the metal and completely reflecting them. As frequencies increase towards the nearinfrared and visible part of the spectrum, field penetration increases, which results in increasing absorption and dissipation. Within this part of the spectrum, metals behave as lossy dielectrics with a complex, frequency dependent dielectric function $\varepsilon(\omega)$. There are various models which can be used to describe the permittivity of metals.

\subsubsection{The plasma model}

The description of the permittivity of metals can be extremely complex. A first order approximation models metals as plasma (free electron gas). EM waves induce electron oscillations with respect to a fixed lattice of positive ions. The oscillations can be characterized by their collision frequency $(\gamma)$ which is inversely proportional to the relaxation time of the free electrons $(\tau=1 / \gamma)$. This model is referred to as the plasma model and ultimately leads to the following expression for the dielectric function of a free electron gas [6]:

$$
\varepsilon(\omega)=1-\frac{\omega_{p}^{2}}{\omega^{2}+i \gamma \omega}
$$


where $\omega_{p}$ is the plasma frequency. The plasma frequency denotes the natural frequency of oscillations of the electrons when they are displaced which is a function of the restoring force of the ion cores. The plasma frequency is given by

$$
\omega_{p}=\sqrt{\frac{n_{e} e^{2}}{m^{*} \varepsilon_{0}}}
$$

where $n_{e}$ is electron density, $e$ is the electron charge, $m^{*}$ is the effective electronic mass and $\varepsilon_{0}$ is the permittivity of free space. The plasma permittivity will have real and imaginary components

$$
\begin{aligned}
& \operatorname{Re}\{\varepsilon(\omega)\}=1-\frac{\omega_{p}^{2} \tau^{2}}{1+\omega^{2} \tau^{2}} \\
& \operatorname{Im}\{\varepsilon(\omega\})=\frac{\omega_{p}^{2} \tau}{\omega\left(1+\omega^{2} \tau^{2}\right)}
\end{aligned}
$$

For $\omega<\omega_{\mathrm{p}}$ the free electron gas behaves as a metal. For frequencies close to $\omega_{\mathrm{p}}$ the permittivity is mostly real and the medium behaves as undamped free electron plasma.

\subsubsection{Drude-Lortentz Model}

The free electron gas model does not match the observed permittivity behavior at and above $\omega_{\mathrm{p}}$. Although the conduction electrons can be modeled as a free electron gas, the metals still have bound electrons. Noble metals (copper, gold and silver) have filled d-bands within their band structure which in conjunction with the background lattice of positive ion cores results in a strong polarization effect. The free-electron model does not account for inter-band electron transitions which drastically increase the imaginary component of the permittivity [6]. The Drude-Lorentz model is a more accurate representation of the permittivity accounting for these polarization effects resulting in a modification of plasma model to [7,8]:

$$
\varepsilon(\omega)=\varepsilon_{\infty}-\frac{\omega_{p}^{2}}{\omega^{2}+i \gamma \omega}+\varepsilon_{\text {int }}(\omega)
$$


in which $\varepsilon_{\infty}$ is a material specific polarization correction factor referred to as the DC optical dielectric constant and $\varepsilon_{\text {int }}(\omega)$ is a fitted permittivity which corrects for the interband transitions, and requires a complicated quantum mechanical derivation. Due to the highly complicated nature of the permittivity models, optical permittivities of metals are typically retrieved from collected experimentally determined values such as in $[8,9]$.

\subsubsection{Surface Plasmon Polaritons}

For most metals the plasma frequency occurs in the visible. Around this frequency, EM waves have appropriate energy to be absorbed by the electrons near the surface and induce a quantized oscillation which is referred to as a plasmon. Plasmons on the surface of a metal are referred to as surface plasmons [10]. These can exist on the surface of a structure such as nanoparticle and are referred to as a localized surface plasmon. This thesis will focus on surface plasmons that exist on semi-infinite planar surfaces between metals and dielectrics which are referred to as surface plasmon polaritons or SPPs. SPPs exist as a coupled excitation between the electron oscillations (plasmon) on the metal surface with the electric field propagating on the dielectric side.

The coupled excitation is depicted in figure 1 . The dielectric has permittivity $\varepsilon_{d}$ while the metal has permittivity $\varepsilon_{m}$. Incident EM waves will induce a coherent oscillation of the surface charges indicated by the "+" and "-" signs which in turn will have associated electric fields indicated by curved the arrows. This wave will propagate along the interface with the propagation constant $\beta_{S P P}$ until it dissipates from resistive losses. 


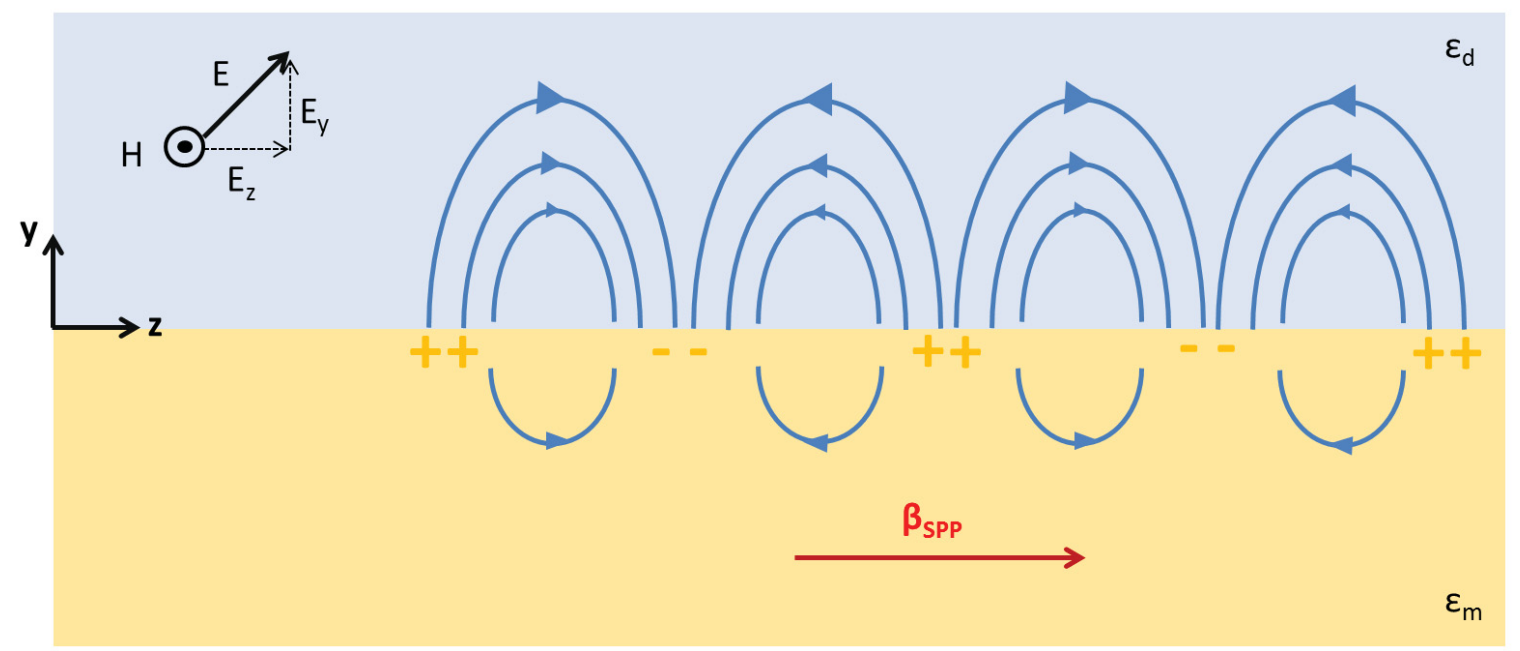

Figure 1 Diagram of coupled excitation between an EM wave (curved lines) and electron surface density waves (+ and -)

The boundary conditions for this situation require that the normal component of the electric displacement field $\left(D_{y}\right)$ needs to be continuous across the boundary such that $D_{y}=$ $\varepsilon_{\mathrm{m}} \mathrm{E}_{\mathrm{y}, \mathrm{m}}=\varepsilon_{\mathrm{d}} \mathrm{E}_{\mathrm{y}, \mathrm{d}}$. Since $\varepsilon_{\mathrm{m}} \neq \varepsilon_{\mathrm{d}}$, this means that there is a discontinuity of the electric fields at the surface which results in surface charges at the interface. Plasmonic metals at these frequencies have a negative permittivity while dielectrics have positive permittivity resulting in a change of direction of the electric field across the interface, which will allow for the propagation of an optical wave coupled to electron oscillations as diagrammed. The field portion in the dielectric is referred to as a polariton and hence this is a surface plasmon polariton. TE polarized waves (s-polarized, E field perpendicular to the plane) have no E field component perpendicular to the surface, thus cannot induce significant polarization, so only TM (p-polarized, E field parallel to the plane of incidence) modes can propagate.

This situation can be described classically by solving the wave equations for a three layer slab waveguide in which the substrate is a metal, the superstrate is a dielectric 
and the waveguide layer has a thickness of 0 . The resulting $\mathrm{E}$ field is an exponential decay on each side of the interface with a dispersion relation obtained as [11]:

$$
\beta=\frac{\omega}{c} \sqrt{\frac{\varepsilon_{d} \varepsilon_{m}}{\varepsilon_{d}+\varepsilon_{m}}}=k \sqrt{\frac{\varepsilon_{d} \varepsilon_{m}}{\varepsilon_{d}+\varepsilon_{m}}}
$$

where $\beta$ is the propagation constant of the SPP, $\varepsilon_{d}$ and $\varepsilon_{m}$ are the complex permittivities of the dielectric and metal respectively and $k$ is the free space wavenumber.

Figure 2 shows various structural implementations of SPP waveguides with overlaid plots of the magnitude of the electric field as a function of $y$. Figure 2 (a) is the simplest situation and is referred to as the single-interface SPP (semi-infinite metal with permittivity $\varepsilon_{m}$ and dielectric $\varepsilon_{d l}$ ). The y-component of electric field peaks at the interface and exponentially decays on each side. In this situation the SPP will propagate along the surface until its energy is dissipated via absorption in the metal (resistive loss) or scattering. This ultimately will result in propagation lengths on the order of 10 to $100 \mu \mathrm{m}$.

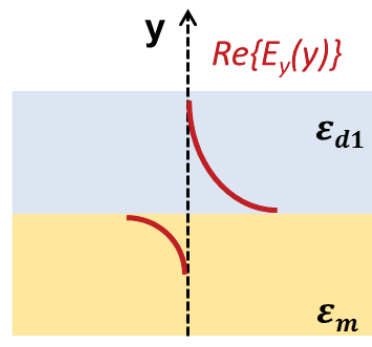

(a)

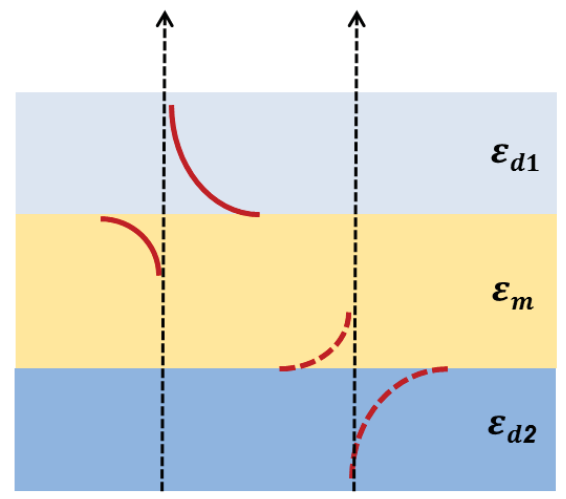

(b)

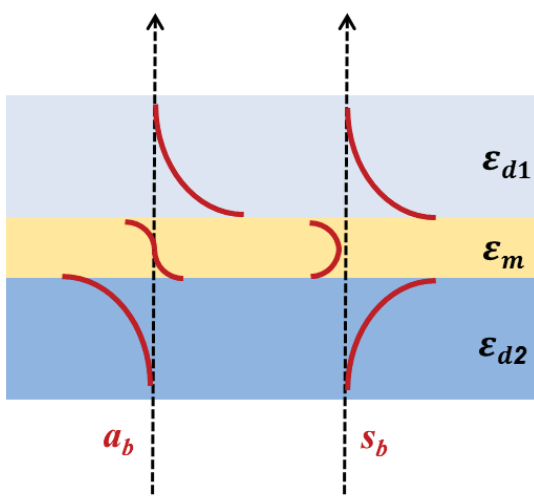

(c)

Figure 2 1-D electric field profiles for (a) Single-interface SPP (b) 2-interface SPPs with large metal thickness (c) coupled SPP modes with thin metal layer

Figure 2 (b) illustrates a finite thickness metal slab with dielectrics on both surfaces. In this case, the metal is significantly thicker than the penetration depth of the field so two 
independent surface modes can be supported. As the thickness of the metal is decreased to figure 2 (c), the two surface modes become bound coupled modes (sometimes referred to as mixed modes or supermodes) and are described by symmetric or asymmetric transverse electric field profiles, and denoted by the nomenclature $s_{b}$ and $a_{b}$ respectively [12].

If the permittivities of the surrounding dielectrics are equal $\left(\varepsilon_{d l}=\varepsilon_{d 2}\right)$ and both are lossless, the symmetric mode will have decreasing attenuation with decreasing metal thickness as the electric field within the metal will be further expelled, whereas the asymmetric mode will have increasing confinement in the metal with decreasing thickness and hence increasing attenuation. The symmetric mode is referred to as a longrange surface plasmon polariton (LRSPP). LRSPP modes have one to two orders of magnitude lower attenuation than their single interface and asymmetric counterparts. The lowered attenuation allows for longer propagation lengths and in terms of sensors, much longer interaction lengths. 


\subsubsection{2-D Plasmon Waveguides}

The situations described so far generally depict 1-dimensional structures (infinite in $\mathrm{x}$ and $\mathrm{z}$ directions, finite in $\mathrm{y}$ ) such as in figure $3 \mathrm{a}$ with a slab of thickness $t$ surrounded by dielectrics $\varepsilon_{d 1}$ and $\varepsilon_{d 2}$. The metal stripe is formed by also limiting the width of the waveguide to a finite value $w$ as in figure $3 \mathrm{~b}$. This allows for the routing and manipulation of SPPs at the cost of reduced confinement. This structure is referred to as the metal stripe.

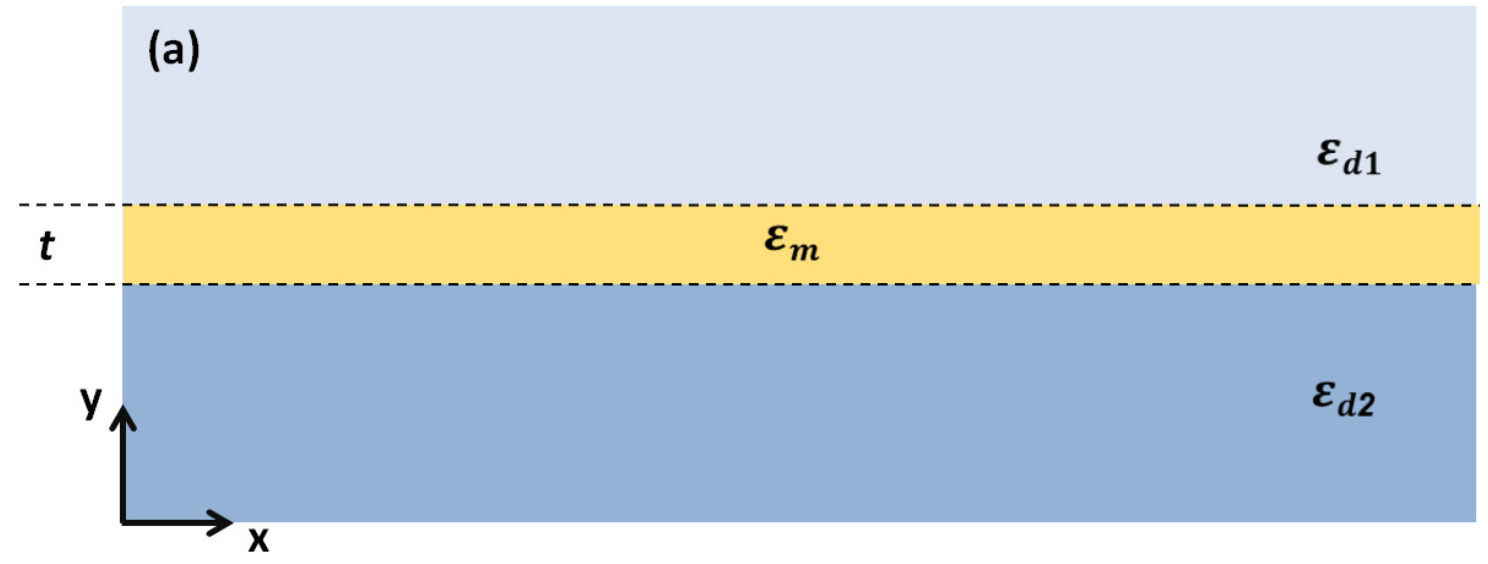

(b)

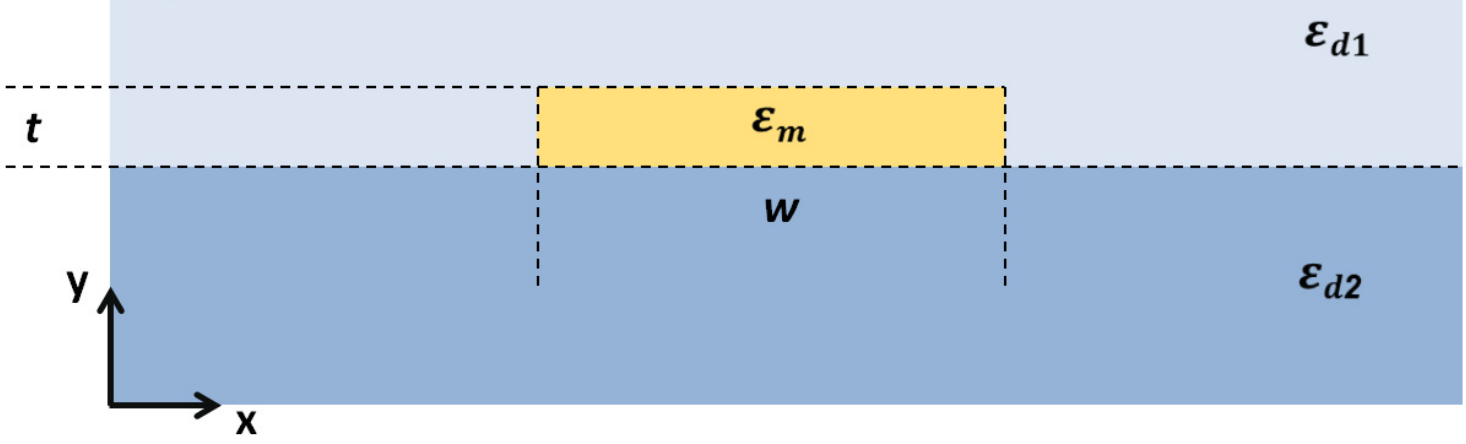

Figure 3 (a) Metal slab waveguide cross-section (infinite in $x$, propagation in $z$ ) (b) Metal stripe of finite width. The substrate and cladding are not necessarily the same, but $\varepsilon_{d 1}=\varepsilon_{d 2}$ is required for LRSPP propagation. 
The width limitation leads to additional lateral confinement which drastically changes the modal solutions. The modal calculations require numerical methods such as the method of lines (MoL), finite element method (FEM) [13] or finite difference method (FDM) [14]. As with the slab case, if the permittivity of the substrate is equal to that of the cladding, then a long range mode can be supported. Another benefit of having a stripe waveguide is that the mode shape can be designed to allow end-fire coupling from a focused TM polarized optical beam. High coupling efficiency can be achieved if the beam profile overlaps the symmetric LRSPP profile.

The previously examined slab waveguide (figure 3 a) has two fundamental modes $\left(a_{b}\right.$ and $s_{b}$ ) which were either asymmetric or symmetric across the x-axis. Assuming $\varepsilon_{d l}=$ $\varepsilon_{d 2}$, the addition of horizontal confinement added in figure $3 \mathrm{~b}$ adds modes which can now be symmetric or asymmetric over the y-axis. This results in 4 fundamental modes, of which the mode that is symmetric along both $\mathrm{x}$ and $\mathrm{y}$ (referred to as the $s s_{b}{ }^{0}$ mode) has the lowest attenuation and is referred to as the LRSPP mode of the stripe waveguide [15]. This architecture allows for the fabrication of passive integrated optical structures such as straight waveguides, bends, splitters, Mach-Zehnder interferometers, etc... [16] which can be structured for functional sensing purposes.

Another structure that can support a LRSPP is diagrammed in figure 4 (a) and is referred to as the membrane-supported metal stripe or simply LRSPP membrane waveguide and has been investigated thoroughly [17-20]. In this situation the metal stripe sits on an ultra-thin dielectric membrane. The surrounding environment can be any dielectric, liquid or gas and self-satisfies the LRSPP requirement of refractive index 
symmetry if the membrane thickness $d \rightarrow 0$. A perturbed LRSPP mode can still be propagated with the membrane waveguide as long as the membrane remains thin such that it is optically non-invasive. As the membrane thickness increases the system becomes increasingly asymmetric with increasing losses.

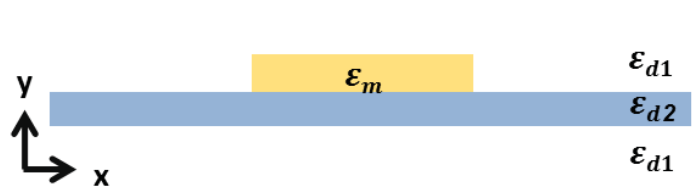

(a)

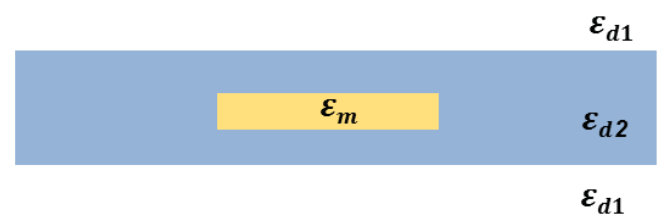

(b)

Figure 4 Cross sections for (a) LRSPP membrane waveguide (b) Cladded membrane waveguide Figure 4 (b) depicts the modified version of the membrane waveguide where the stripe is embedded symmetrically into the membrane. This situation is referred to as the cladded membrane waveguide and its design, fabrication and testing is the primary focus of this thesis. This structure has increased symmetry over the membrane waveguide and hence improved propagation lengths, along with greater mechanical strength.

\subsubsection{Modal Studies of Plasmon Interfaces}

The propagation of SPPs can be investigated by solving Maxwell's equations. The simplest situations involve the 1-D structures depicted in figure 2. Assuming no external charges or current densities and homogenous permittivities, the solution can be determined by solving the wave equation in each region and matching fields using the appropriate boundary conditions. For 2-D structures as described in the previous section, the permittivity becomes a function of $\mathrm{x}$ and $\mathrm{y}$ and is represented by the complex function 
$\varepsilon(x, y)$. Assuming a time harmonic propagating solution, the vector wave equation for the electric field $\mathbf{E}$ and magnetic field $\mathbf{H}$ are expressed as [15]:

$$
\begin{aligned}
\nabla \times \nabla \times \mathbf{E}-\omega^{2} \varepsilon(x, y) \mu \mathbf{E} & =0 \\
\nabla \times \varepsilon(x, y)^{-1} \nabla \times \mathbf{H}-\omega^{2} \mu \mathbf{H} & =0
\end{aligned}
$$

where the magnetic permeability $\mu$ is assumed to be homogenous and equivalent to $\mu_{0}$. It is assumed that the waveguides are invariant along the direction of propagation (z) such that the $\mathrm{z}$ dependence is $e^{-\gamma z}$ where the propagation constant is defined as $\gamma=\alpha+i \beta$ and $\alpha$ is the attenuation constant and $\beta$ is the phase constant. The solutions to the above equations represent the supported modes of the structure.

The modal solutions for 2-D cross sections require numerical approaches. For this thesis a large series of designs needed to be explored with various geometries, thus commercial FEM software (Comsol Multiphysics) was used for the design as discussed in Chapter 3 and Chapter 4.

\subsubsection{Excitation Techniques}

The excitation of SPPs requires the phase matching of the propagation constants of the input light $(k)$ to that of the SPP $(\beta)$. Considering the single interface SPP, equation 6 shows that $\beta$ will always be larger than $k$ and therefore direct excitation of the SPP is not possible. Figure 5 plots the dispersion relation [6] of a single interface SPP with the solid line representing $k_{0}$ and the dashed red line showing $\beta_{S P P, a i r}$. Excitation is often achieved using a configuration referred to as the Kreschmann geometry diagrammed in Figure 5 where a thin metal film is evaporated onto a $\mathrm{SiO}_{2}$ prism of refractive index $n_{p}$ as diagrammed. If the input light is propagating in a high refractive index medium as 
represented by the solid blue line, with the propagation constant $k_{p}$ and at an angle $\theta$ greater than the critical angle, evanescent coupling to the SPP along the air/metal interface is possible. Although a SPP could also propagate along the metal/prism interface, it cannot be excited in this geometry. The prism coupling structure can be modified by placing an additional dielectric between the metal and the prism and or using a different dielectric on top of the metal.

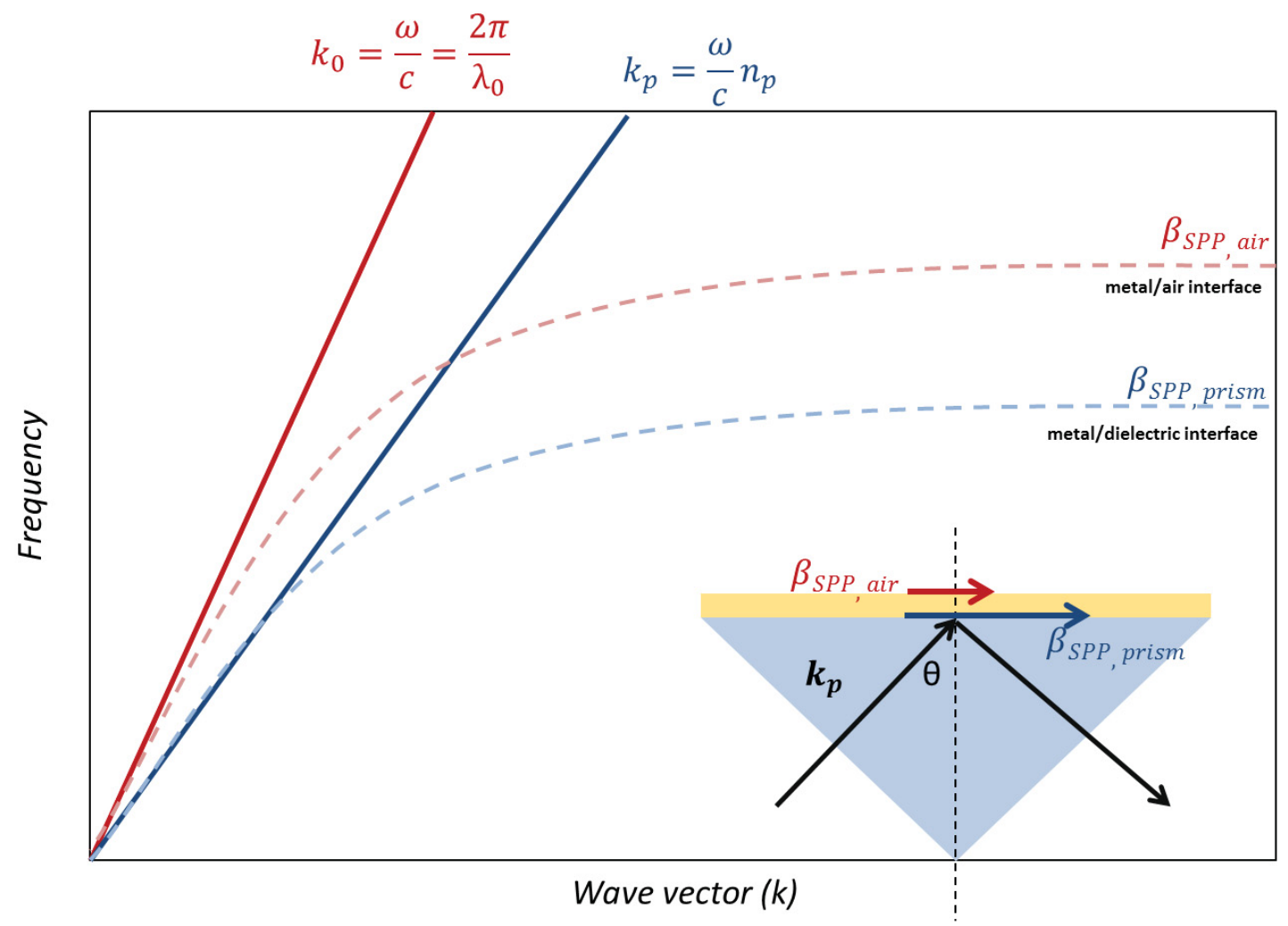

Figure 5 Dispersion relation for a single interface SPP

Although prism coupling is fairly simple, it can be somewhat bulky and often impractical. Another method of exciting SPPs and LRSPPs is grating couplers. A periodic grating (either bumps or trenches) with a separation of $\Lambda$ is diagrammed in Figure 6. Although this diagram shows the periodicity in the metal, it could also be 
patterned in the dielectric portion or made from another material. These couplers work by scattering incoming light with propagation constant $k_{0}$ at incident angle $\theta$. The component of the scattered light which matches the propagation constant of the SPP will couple that energy into the waveguide.

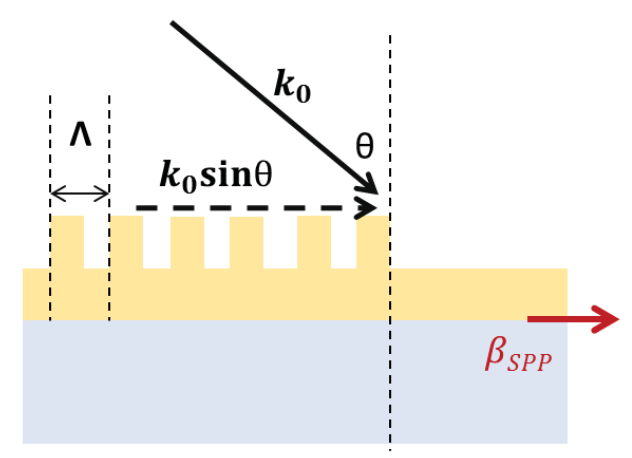

Figure 6 Example of a grating coupled SPP shown as a step-in-height of the metal

Phase matching can occur if :

$$
\beta=k \sin \theta \pm m \frac{2 \pi}{\Lambda}
$$

where $\mathrm{m}$ is any integer. The benefit of grating couplers is that they can be tuned to the propagation constant of a specific mode (i.e. the LRSPP mode if supported), however due to the scattering nature of these structures, the coupling efficiency is limited. The scattering mechanism works in reverse such that a grating can also be used to out-couple light from a propagating SPP.

\subsubsection{Plasmon Sensors}

The electric fields of SPPs peak at the surface of the metal. This property means that changes along the metal surface will produce changes in the field which will result in significant changes in the propagation constant. This property is the main reason for the 
application of SPPs to sensing. Generally, these sensors use a structure such as one of those previously discussed, with the addition of a transduction medium on the surface which can be exposed to a particular analyte that will induce a shift in the plasmon behavior.

Biosensing is one of the primary applications in this field. Typically gold is used as the metal due to the fact that it has been well characterized and can also be easily functionalized through known surface chemistries. A Kreschmann configuration can be used where the metal surface is functionalized with immobilized molecules such as antibodies. Exposure of this surface to a medium which contains the corresponding antigen would form a concentration dependent adlayer due to binding which would in turn cause a change in the effective permittivity of the metal resulting in a shift of the plasmon resonance angle. The LRSPP stripe waveguide biosensor has been demonstrated [21] in which a stripe waveguide is embedded in a low refractive index polymer (Cytop). A microfluidic flow channel is used with index matched carrier fluid. Many other implementations have been presented for biosensing [22].

Although biosensing is a major application, gas sensing is also of particular interest and benefits from the same strengths as biosensors in that they can be highly sensitive and compact and in many cases simpler to implement since less surface preparation is required. This dissertation will focus on the development of a hydrogen $\left(\mathrm{H}_{2}\right)$ gas sensor based on the LRSPP cladded membrane waveguide structure introduced previously. 


\subsection{Hydrogen Sensors Review}

\subsubsection{Hydrogen Gas}

Hydrogen $(\mathrm{H})$ is the simplest and most abundant element in the universe. At standard temperature and pressure it exists as a combustible diatomic gas $\left(\mathrm{H}_{2}\right)$. However, it is highly reactive and readily forms covalent bonds with most non-metallic elements and molecules and hence pure $\mathrm{H}_{2}$ occurs very rarely under natural planetary conditions. The $\mathrm{H}_{2}$ content of the atmosphere (air) is $\sim 0.55 \mathrm{ppm} \mathrm{[23].} \mathrm{H}_{2}$ is used in chemical processing such as petroleum refinement as well as for various other industrial purposes. Hydrogen is also an energy carrier with the capability of being an efficient emissions-free fuel source in the form of fuel cells. Although commercial hydrogen sensors currently exist, the growing hydrogen economy has resulted in the need for more efficient, faster, safer and more reliable sensors.

\subsubsection{Palladium}

Palladium $(\mathrm{Pd})$ is element 46 of the periodic table and is a fairly rare transitional metal. It has been observed that Pd (as well as other transition metals) has a strong ability to proportionally absorb hydrogen, forming palladium hydride $\mathrm{PdH}_{\mathrm{x}}$ where $\mathrm{x}$ is the atomic ratio of hydrogen to palladium. This has led to significant research into Pd for uses in hydrogen storage and hydrogen purification [24]. $\mathrm{H}_{2}$ adsorbs onto the Pd surface which disassociates the molecule into two $\mathrm{H}$ atoms which then diffuse into the Pd lattice [25]. The process is generally reversible which makes Pd a one of the best materials to use in $\mathrm{H}_{2}$ sensors. Pd has a face-centered cubic (FCC) crystal structure which allows hydrogen to rapidly diffuse through the lattice. The $\mathrm{H}$ atoms will occupy random 
octahedral interstitial positions within the FCC lattice. $\mathrm{PdH}_{\mathrm{x}}$ up to $\mathrm{PdH}_{0.02}$ is referred to as the $\alpha$-hydride phase as diagrammed in figure 7 .

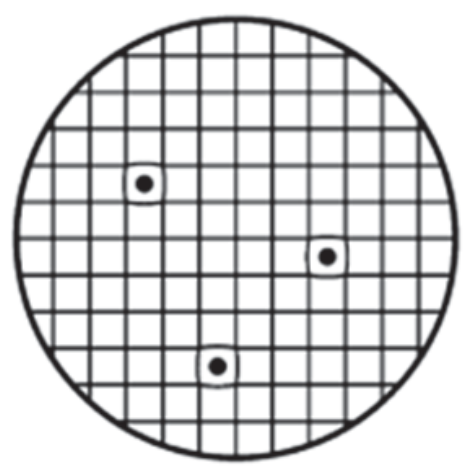

$\alpha$

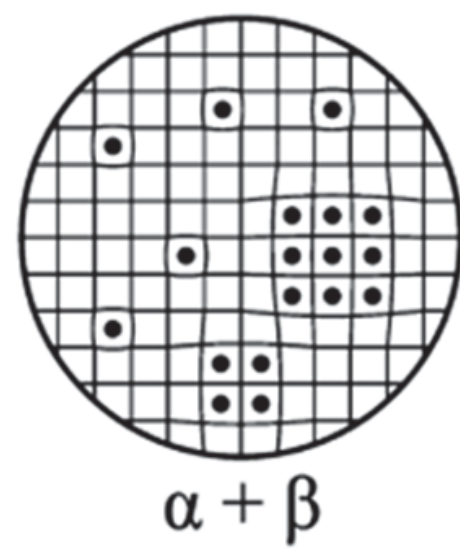

Figure 7 Diagram of hydrogen atom distribution with in the Pd lattice for the various hydride phases. Reprinted (adapted) with permission from [26]. Copyright 2014 American Chemical Society.

At high concentrations of $\mathrm{H}, \mathrm{Pd}$ will saturate and $\mathrm{H}-\mathrm{H}$ attraction will result in an ordered and uniform distribution referred to as the $\beta$-hydride phase. For concentrations in between there is a transitional region where both phases exist, referred to as the $\alpha+\beta$ phase. The addition of $\mathrm{H}$ atoms in the $\alpha$ phase induces a mild and often inconsequential lattice expansion from $3.887 \AA$ for $\mathrm{Pd}$ to $3.895 \AA$ for $\mathrm{PdH}_{0.02}$. However the $\beta$ phase produces a significant expansion to $4.025 \AA$ [27] with a corresponding volume expansion of the metal.

The kinetics of the hydride phase formation are temperature dependent and commonly represented by a plot known as the pressure-composition-temperature (p-c-T) plot also referred to as pressure-composition isotherms. Figure 8 (a) shows a diagram of the PdHx isotherms. 


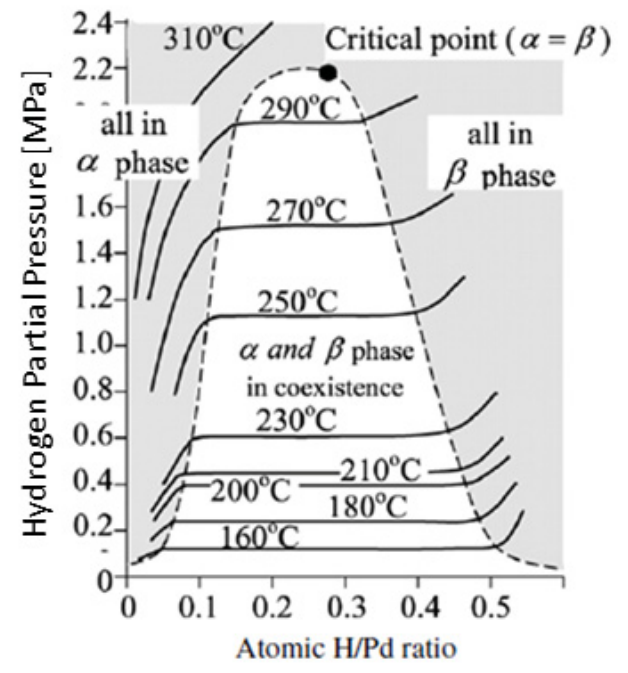

(a)

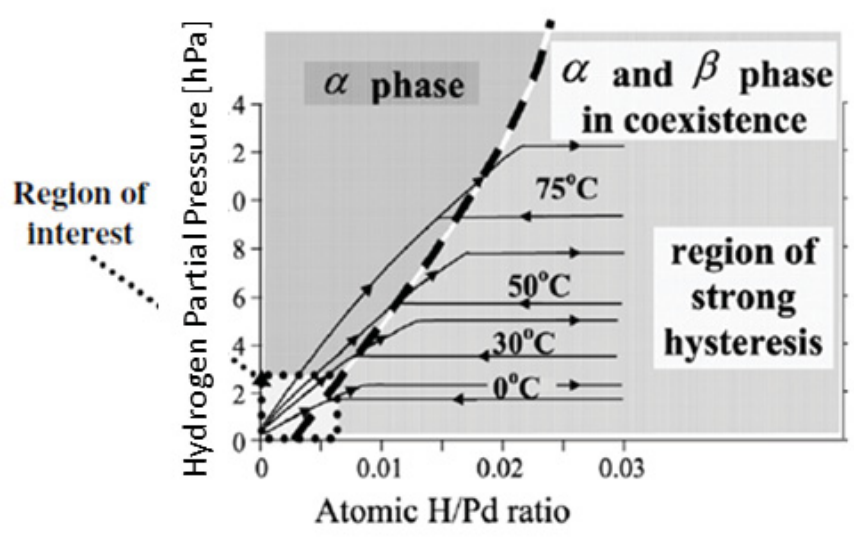

(b)

Figure 8 (a) Pressure-composition-isotherms for Pd and (b) magnified view of the operating range of interest. Modified with permission from [28]. Copyright 2007 IOP Institute of Physics.

The isotherms plot hydrogen pressure (equivalent to concentration) as a function of the atomic $\mathrm{H} / \mathrm{Pd}$ ratio for various temperatures. It is seen that above $\sim 300{ }^{\circ} \mathrm{C}$ the mixed phase disappears. As the temperature is lowered the mixed phase widens and ultimately saturates at lower concentrations. The importance of this plot is that absorption and desorption from the mixed region causes significant lattice strain which can lead to hysteresis and irreversible mechanical damage to the Pd.

Most hydrogen sensors tend to operate in the lower concentration ranges and in general, at lower temperatures as highlighted in figure 8 (b). Under these conditions transition into the mixed region, even for reasonably low concentrations needs to be considered.

Absorption of $\mathrm{H}$ into the Pd lattice directly affects the permittivity as defined in equations 2-4. The addition of the hydrogen atoms increase the electron density as well as the collision frequency which would result in an expected increase in the plasma 
frequency and thus increase of the imaginary component of the permittivity (increased attenuation). However, the absorption also causes a lattice expansion which, depending on other conditions, can result in a decrease of the electron density despite the addition of the corresponding electron, which would thus lead to a decrease in attenuation. This behavior is observed when comparing optical $\mathrm{H}_{2}$ sensors as some experience increases in attenuations why others experience decreases.

\subsubsection{Sensor and detection overview}

The United States Department of Energy has outlined target specifications for ideal hydrogen sensors [29]. The specifications are: a measurement range of $0.1 \%-10 \% \mathrm{H}_{2}$ concentration, operating temperature between $-30{ }^{\circ} \mathrm{C}$ to $80{ }^{\circ} \mathrm{C}$, less than 1 second response time, $5 \%$ full scale accuracy, operation in ambient air, and over humidity ranges from $10 \%-98 \%$, a 10 -year lifetime and no cross-sensitivity. To date no sensor has been able to meet all of these requirements. This largest challenge to date is to meet the desired sub-second response time. Although some sensors have demonstrated extremely quick responses, they do so at the cost of a reduced measurement range.

There are many techniques that have been used each having specific strengths and drawbacks. Catalytic sensors utilize the high reactivity of hydrogen and oxygen on a sensor surface to detect hydrogen levels. This technique has been used to detect many types of combustible gases. A simple pellistor-type sensor uses a heated coil coated with a catalyst (i.e. Pd) which detects shifts in resistance. These types of sensors are quite commonly available in the market including modernized structures [30]. The major benefit of catalytic sensors is that they can have very low detection limits and good 
sensitivity and response times. These sensors typically have problems with selectivity, specifically to other hydrocarbons. They also rely on the presence of oxygen. Additionally, sensors that contain heating elements have larger power consumption and can be inherently dangerous (i.e., they can ignite $\mathrm{H}_{2}$ ).

Semiconductor-based sensors have also been studied [31] and are commercially available. Pd film-based structures such as Schottky diodes, MOSFETs and MOS capacitors translate changes in work function, resistivity, and conductivity into corresponding voltage/current/capacitance signals. Temperature has a large influence on the response of semiconductor sensors, so the sensor temperature needs to be controlled carefully. Additionally, electronic devices typically operate with moderate currents or voltages which pose an ignition risk.

An interesting alternative to the aforementioned sensors is to use optical techniques. The primary benefit is the removal of any heating or electrical elements from the environment. These sensors depend on changes to the optical properties of palladium instead of a temperature/resistance change due to an oxygen reaction, which means optical sensors do not require oxygen to operate. Additionally, optical sensors are less temperature sensitive since they only have to account for thermal expansion whereas semiconductor sensors have strong conductivity changes with temperature.

One of the first optical based sensors was presented by Butler [32] and utilized a simple implementation consisting of a palladium coated tip (micromirror) on the end of a cleaved optical fiber and measuring the change in reflectivity upon hydrogen uptake. Other sensors have also expanded on this concept [33,34]. Fiber Bragg gratings (FBGs) are periodic refractive index modulations in the core of an optical fiber core which 
produce sharp wavelength peaks in the reflection/transmission spectrum. There are various demonstrated implementations of Pd with FBGs [35-38], as well as coated optical fibers [39, 40], tapered fibers [41, 42] and fiber lasers [43]. Other waveguide structures have also been demonstrated such as a planar silicon on insulator (SOI) waveguide based sensor [44]. Fiber sensors and planar waveguide sensors can be useful for sensing, especially over longer propagation ranges however since the electric fields are confined within the waveguide, the sensitivity may not be as high as other methods of detection. Plasmonic based sensors based on Pd confine the E-field directly on the Pd surface which can greatly increase sensitivity. Localized surface plasmon resonance (LSPR) has been demonstrated in various implementations, particularly in the form of Pd nanoantennas or nanodisks [45]. SPR-based optical sensors fit nicely into this regime as they provide the benefits of optical detection while also having strong surface sensitivity from the plasmonic response of $\mathrm{Pd}$ under $\mathrm{H}$ absorption.

\subsubsection{Plasmonic Hydrogen Sensors Review}

The strong optical response of Pd to $\mathrm{H}$ implies that plasmonic sensors may be ideally suitable for this task. Figure 9 shows a summary of previously demonstrated SPP-based hydrogen sensors. The Kreschmann geometry is one of the simplest methods of exciting SPPs and hence it was used first by Chadwick et al. [46] using a Pd coated prism. This method was further refined by Konopsky [47] by adding in a 1D photonic crystal (1DPC) between the Pd film and prism to create an effective symmetry to propagate LRSPPs. Evanescent SPR coupling of coated optical fibers have been demonstrated by Bevenot [48] with a Pd coated core of a multimode fiber while Perrotton [49] used a $\mathrm{Pd} / \mathrm{SiO}_{2} / \mathrm{Au}$ 
coating. Tobiska [50] demonstrated a Pd coated single mode planar channel waveguide made of glass.

(a)

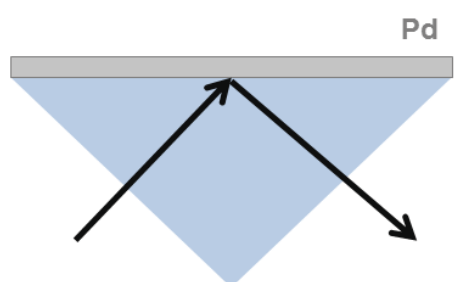

(c)

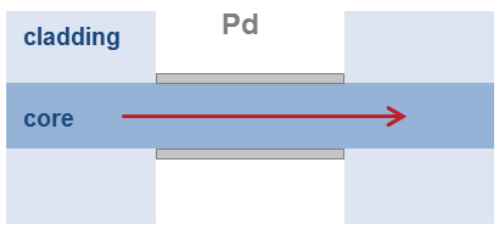

(e)

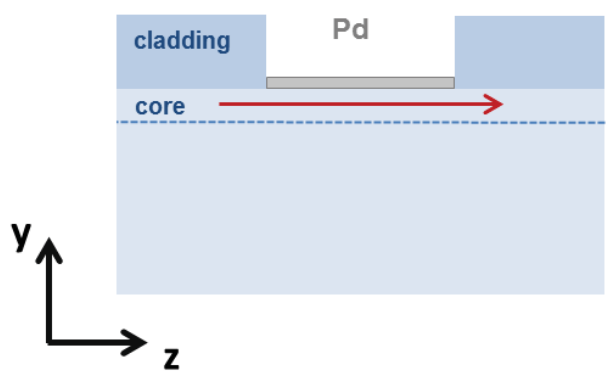

(b)

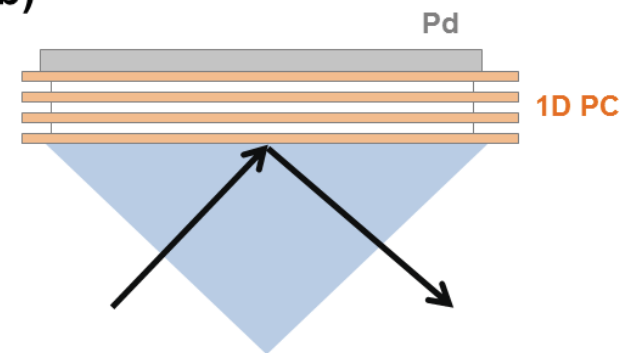

(d)

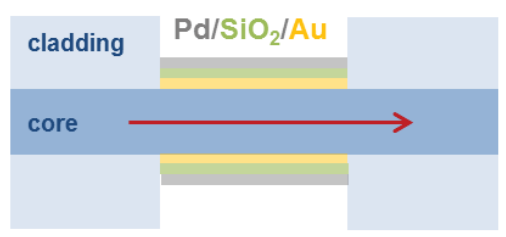

Figure 9 Various SPR hydrogen sensors (a) Kreschmann geometry [46] (b) LRSPP Kreschmann with 1D PC [47] (c)-(d) Pd coated fibers [48,49] (e) Pd coated channel waveguide [50]

The Kreschmann structures are inherently bulky which makes them difficult to implement in practice. Fiber-based sensors provide easy optical coupling but can be challenging to fabricate since most fabrication tools and techniques are designed for planar structures. Planar optical waveguides provide the ability to fairly easily couple light through end-fire coupling, grating couplers or prism couplers, and can be fabricated 
using standard microfabrication techniques. Although planar devices may require more fabrication steps compared to fibers or prisms, the cost per device of is greatly decreased since a single wafer can yield hundreds of devices whereas non-planar structures cannot be easily mass produced.

\subsection{Materials and Microfabrication}

One of the most influential factors involved with the proliferation of plasmonic technologies is improvements in nanofabrication materials and technologies. By taking advantage of traditional planar fabrication techniques, plasmonic devices, and specifically plasmonic waveguides, can be manufactured at the wafer level, which ultimately can allow for mass production. The evolution of semiconductor fabrication is primarily motivated by increasing miniaturization of integrated circuits for complementary metal-oxide-semiconductor (CMOS) devices. Many of the techniques that have been developed can be modified for fabricating integrated optical devices. This section overviews the relevant background related to the fabrication of the membrane waveguides.

\subsubsection{Spin-coated polymers}

Polymers play multiple important roles in microfabrication and micromachining. They are typically flexible, easily molded and chemically selective materials which allow them to be used in building microstructures. Some commonly used polymers are Polydimethylsiloxane (PDMS), Poly(methyl methacrylate) (PMMA) and Polyimide which have been used for microfluidics [51]. Similarly, most resists (photoresists, e-beam resists) are also amorphous polymers. Different polymers each have various mechanical 
and optical properties which allow them to serve distinct purposes. In most applications a thin film polymer ( $2 \mu \mathrm{m}$ or less) layer is required and the most common method of application is through spin coating [52]. Most polymers used in microfabrication are diluted in a solvent to facilitate spin coating where the liquid state polymer is applied to a flat surface which is rotated about its center axis. The angular speed of the rotation along with the viscosity of the polymer will determine the thickness of the film.

Most amorphous polymer films require a curing stage, which refers to a hardening of the polymer via evaporation of the solvent. This is typically done by heating the material at temperatures above the material's glass transition temperature $\left(T_{g}\right)$. Above $T_{g}$ the molecular chains have large thermal energy and minimal resistance to movement and hence this is referred to as the rubbery region. Below $T_{g}$, polymers solidify and become rigid and are referred to as glassy. Certain polymers exhibit the ability to cross-link chains when they are first brought above $T_{g}$ and then returned to the glassy state. This can result in the new glassy polymer being solvent resistant.

\subsubsection{Cytop}

Cytop is an amorphous (non-crystalline) fluoropolymer developed by AGC Chemicals and has been used extensively as the primary dielectric for planar LRSPP devices [53-55]. The polymer formula is described in Fig 10 [56].
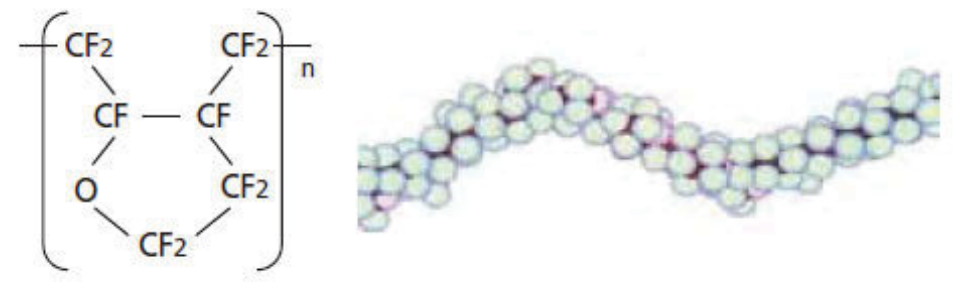

Figure 10 Molecular formula for Cytop polymer 
The fluoropolymer nomenclature refers to the multiple Carbon-Fluorine bonds which result in high chemical resistance, specifically to acids and bases. Cytop shares similar chemical resistance to Polytetrafluoroethylene (PTFE - Teflon) however its amorphous, non-crosslinking nature compared to the crystalline structure of PTFE gives Cytop extremely high optical transmission over a broad spectrum and a low $(\sim 1.34)$ refractive index. This property combined with high chemical resistance makes Cytop highly desirable for optical sensors. In this project Cytop is used as the dielectric for the membrane waveguide. The low refractive index allows for thicker and more mechanically robust membranes. For hydrogen sensing, a Cytop substrate provides a non-rigid surface that limits clamping of Pd films which is discussed further in Chapter 7.

There are several grades of Cytop (Type A, M and S) each with different polymer end-groups which provide different properties. A-grade has high transparency in the visible spectrum, M-grade contains an amino-silane coupling agent which promotes good adhesion to metals and glass without an additional adhesion promoter. S-grade is referred to as the optical grade and has the largest transparency spectrum from deep UV through near infra-red.

Cytop can be applied most easily through spin-coating. It has a proprietary solvent (CT-Solv) and a $\mathrm{T}_{\mathrm{g}}$ of $108^{\circ} \mathrm{C}$. However, as Cytop does not cross-link when it is in its glassy phase, it is not as physically rigid as other materials can be. Similarly if it is brought back above $T_{g}$ after solvent evaporation, significant reflow can occur. Some of the mechanical properties of Cytop thin films have been explored in [57]. 


\subsubsection{Metallization of LRSPP waveguides}

To form a LRSPP waveguide, a method to deposit and pattern the metal on a substrate is required. From traditional microfabrication techniques there are two primary methods: direct etching and lift-off. With direct etching a blanket metal layer is deposited on a surface and then it is masked (typically with a photoresist) and selectively etched via either wet or dry methods. However direct etching requires material specific etch techniques and is less commonly used when fine features are required.

Lift-off, or bi-layer lift-off is a process more commonly used when features with sharply defined edges are required. This process is outlined in Figure 11.

(a)

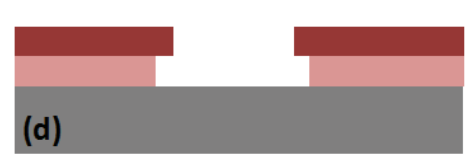

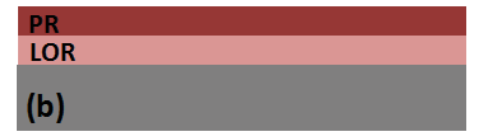

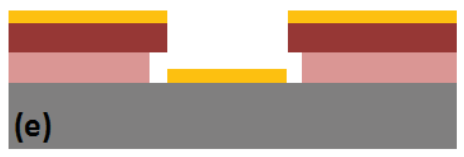

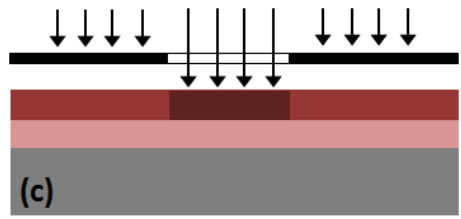

(f)

\section{Figure 11: Overview of bi-layer lift off metallization}

In this technique a non-photo-sensitive lift-off resist (LOR) is applied on the substrate followed by a normal photoresist (PR) as in Figure 11b. The features are patterned through optical lithography (Figure 11c) and developed. When developed, the exposed area of the photoresist is selectively removed while the LOR is isotopically removed forming an undercut (Figure 11d). A blanket metal can be deposited (Figure 11e). Finally the LOR layer is dissolved with a wet etch which subsequently removes the remaining PR and unwanted metal leaving only the desired pattern. Metallization on silicon or silicon dioxide is fairly comment and straightforward however metallization on 
Cytop is a more complicated process that has been developed and refined in [58-60] as well as in Chapter 5 of this thesis.

\subsubsection{Electron-beam Lithography}

Photolithography systems have practical limitations to the smallest resolvable dimension. This is caused by the diffraction limit of the exposure feature size on the photomask and the wavelength of the exposure light. There are techniques to work around these limits such as using progressively shorter wavelength exposure sources or diffraction masks, however they can be quite costly and ultimately less effective than simply moving away from optical lithography.

One technique that has been used to accurately define extremely small $(<10 \mathrm{~nm})$ and precise features has been electron-beam lithography (EBL) [61]. In this technique high energy electrons (10-100 keV) are focused onto electron sensitive resists which can then be used to define precise features. For example, grating couplers are highly dimensionally sensitive so e-beam definition is often a necessity. However e-beam lithography has drawbacks such as slow exposure speeds and high system cost. Also, exposure on insulating substrates can cause resolution issues due to charge buildup. In the case of insulating substrates, a charge dissipation layer can be applied as either a metal over-layer or conductive polymer layer.

\subsubsection{Silicon wet etching}

Microstructures such as membranes are often created by depositing or growing a thin film on a substrate and then selectively removing that substrate. In the case of the 
membrane waveguide, the film is Cytop which can be spin-coated. The substrate material is arbitrary, however silicon is often used as it has been studied extensively with wellknown masking and etching techniques. Dry etching includes techniques such as reactive ion etching (RIE) and deep reactive ion etching (DRIE) which can anisotropically remove silicon. These techniques can be used to create large aspect ratio trenches in silicon but can be somewhat aggressive. Wet techniques have the benefit of being chemically selective with the two most common chemicals for etching silicon being potassium hydroxide $(\mathrm{KOH})$ and tetramethylammonium hydroxide (TMAH). These two chemistries are preferred as they can be easily masked by $\mathrm{SiO}_{2}$ or $\mathrm{Si}_{3} \mathrm{~N}_{4}$ masks. Both chemistries are equally viable and well-studied $[62,63] . \mathrm{KOH}$ can produce faster etch rates however if potassium contamination is an issue, then TMAH is preferable.

\subsection{Project development history}

The history and development of LRSPP waveguides in Cytop is diagrammed in figure 11. The original LRSPP membrane waveguide was introduced in [17-20] as a Au stripe suspended on a silicon nitride $\left(\mathrm{Si}_{3} \mathrm{~N}_{4}\right)$ membrane of thickness $\mathrm{d} \sim 1 \mathrm{~nm}$. In concept, the device in figure 11 (a) could be used in any environment (gaseous or liquid) due to inherent near-symmetry around the membrane. Realistically, only higher index mediums (i.e. water) can be used due to the strong perturbation caused by the nitride $(\mathrm{n} \sim 2.0)$. This architecture was studied primarily in the context of biosensing as the typical medium for this would be water or carrier fluids closely matched to water.

There were several impracticalities with this setup. Firstly, the high refractive index of nitride meant that the membrane would need to be excessively thin. Nitride films 
on silicon exhibit strong tensile stress which results in membranes being highly fragile. In addition, adhesion of $\mathrm{Au}$ to nitride is poor and a chrome $(\mathrm{Cr})$ adhesion layer was required which increased losses. The search for an alternate membrane material led to Cytop, which has a refractive index that is closely matched to water $(\sim 1.34)$, and allows for the fabrication [58] of significantly thicker membranes which were more mechanically robust [57]. Au can also be applied directly to Cytop without additional adhesion materials. As a biosensor, the matched refractive index of Cytop to water logically leads to the conclusion that a membrane is not necessary to maintain refractive index symmetry as an optically infinite Cytop substrate can be used instead as in figure 11 (c). A large family of LRSPP devices of Au stripes on thick Cytop substrates have been explored [50]. Further practical implementation was achieved with the fabrication of microfluidic channels as in figure 11 (d) [60] which has resulted in fully operational biosensor chips [21]. 


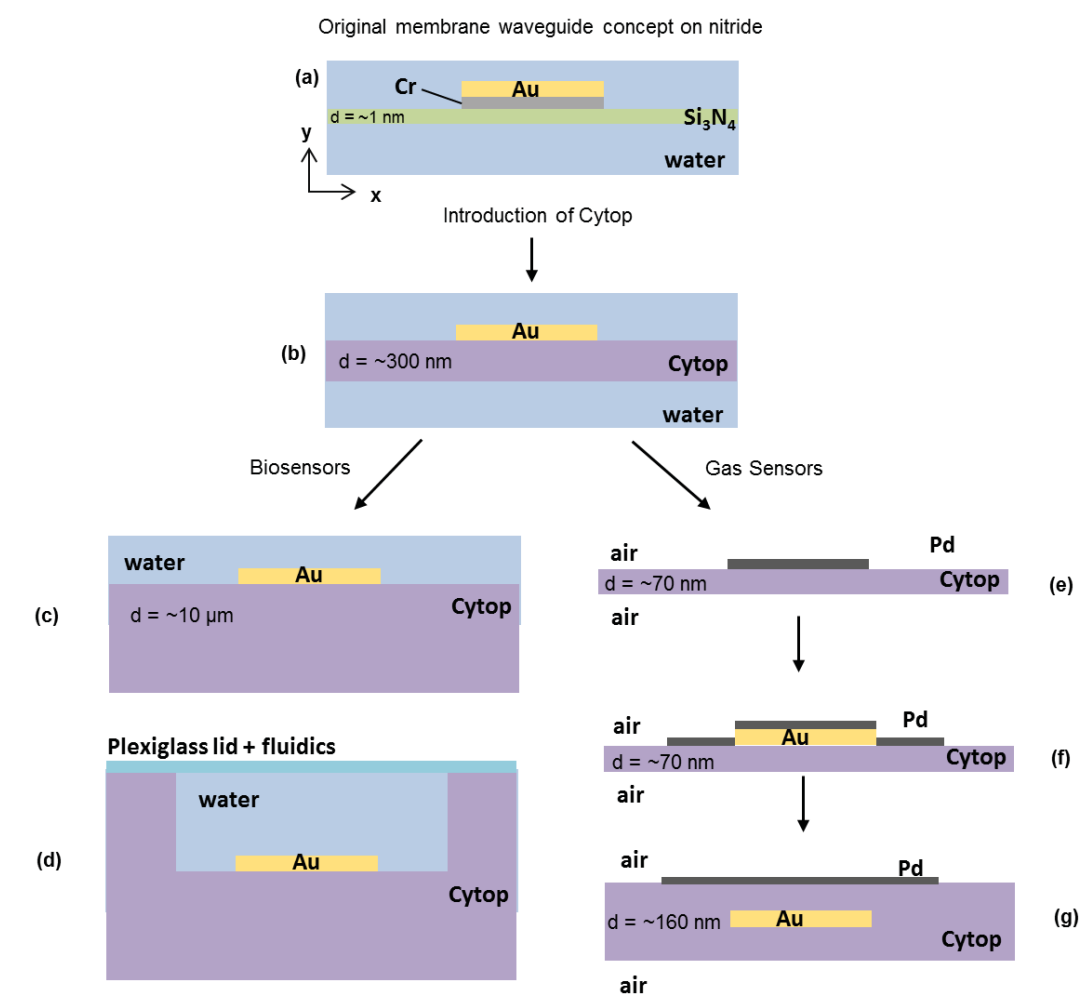

Figure 12 LRSPP waveguide history (a) original membrane waveguide on nitride [20] (b) membrane waveguide on Cytop [58] (c) Uncladded LRSPP biosensor waveguide [59]. (d) LRSPP biosensor waveguide with microfluidic channels [60] (e) First generation membrane waveguide hydrogen sensor - CH4 (f) Second generation (g) Cladded membrane waveguide hydrogen sensor CH5

The membrane waveguide with Cytop however allows for feasible operation as gas sensor operating in air. Hydrogen gas sensing was chosen in particular due to the relative material simplicity of using $\mathrm{Pd}$ as a transducer. The first generation hydrogen sensor is diagrammed in figure 12 (e) a simple membrane waveguide consisting of a Pd stripe on Cytop membrane. The design of this implementation is covered in Chapter 3. A Pd-only waveguide resulted in extremely high optical losses. It was decided to instead utilize a lower loss $\mathrm{Au} / \mathrm{Cytop}$ membrane waveguide with small functionalized regions with Pd overlays as diagrammed figure 12 (f). The overlay was designed to be wider than the Au stripe to simplify the alignment during fabrication. Although this structure 
presented significantly lower losses than the Pd stripe, the membrane thickness was still limited due to the need for refractive index symmetry. It was finally concluded that the optimal design would be the cladded membrane waveguide in figure $12(\mathrm{~g})$ and discussed in detail in Chapter 4. Figure 13(a) shows the computed electric field profile through a cross section of a Pd covered membrane waveguide (as in Figure 12g). Figure 13 (b) shows the magnitude of the electric field as a function of y-direction through the center of the structure. It is shown that the electric field will favor the palladium patch and thus more field is confined to the Pd surface than the $\mathrm{Au}$, which is desirable for increased sensitivity to hydrogen. This structure has drastically improved symmetry which results in lower losses, while simultaneously allow for a thicker and stronger membrane.

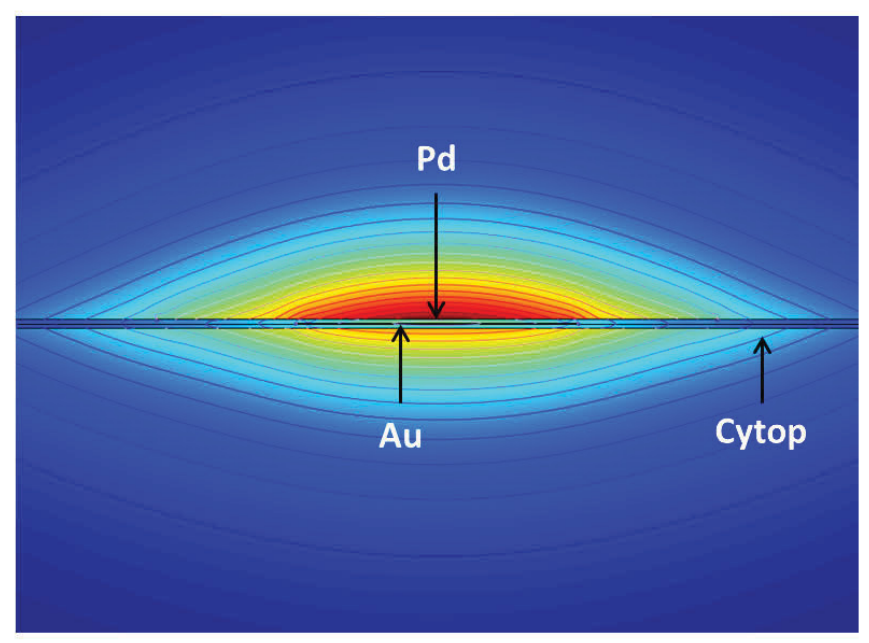

(a)

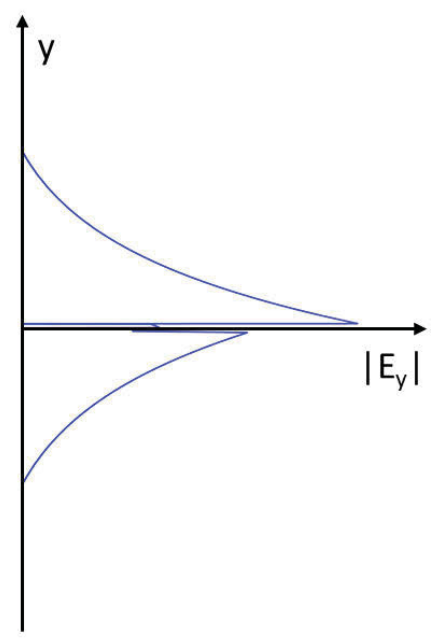

(b)

Figure 13 (a) Electric field (y-component) distribution over the cladded membrane waveguide hydrogen sensor cross section (b) Electric field distribution through the structure center showing maximum electric fields along the Pd/air interface 
The thick Cytop substrate structures can be excited via end-fire coupling from cleaved optical fibers as the mode profiles can be overlapped. This coupling requires precisely flat optical end facets which can be achieved through wafer dicing. Although theoretically possible with a membrane structure, obtaining a sharp end facet either via dicing or cleaving of a membrane is very difficult. For Cytop membranes, cleaving a flat edge is difficult since the membrane will tend to stretch and deform before breaking. In addition, removing an edge of a membrane will induce non-uniform stress and lead to mechanical instability. It was decided to use grating couplers to excite the LRSPPs. This method allows for perpendicular excitation while also maintaining the structural integrity of the membrane. 


\section{Chapter: Modeling of Plasmonic Hydrogen Gas Sensors}

The following article entitled "Modeling and design of hydrogen gas sensors based on a membrane-supported surface plasmon waveguide" was published in Sensors and Actuators B: Chemical volume 161 in January 2012 and is presented in full.

The article represents work performed solely by the primary author under the supervision and guidance of the co-authors. It covers the modeling and design theory of the first generation LRSPP membrane waveguide hydrogen sensor. In this implementation a Pd waveguide is proposed and two architectures (straight waveguides and MZIs) are compared. This design was ultimately not used, however the principles presented lead to the final device as presented in Chapter 4. 


\title{
Modeling and design of hydrogen gas sensors based on a membrane-supported surface plasmon waveguide
}

\author{
Norman R. Fong ${ }^{\mathrm{a}, *}$, Pierre Berini ${ }^{\mathrm{b}, \mathrm{c}}$, R. Niall Tait ${ }^{\mathrm{a}}$ \\ a Department of Electronics, Carleton University, 1125 Colonel By Drive, Ottawa, ON, Canada K1S $5 B 6$ \\ ${ }^{\mathrm{b}}$ School of Electrical Engineering and Computer Science, University of Ottawa, 161 Louis Pasteur, Ottawa, Ontario, Canada K1N 6N5 \\ ${ }^{c}$ Department of Physics, University of Ottawa, 161 Louis Pasteur, Ottawa, Ontario, Canada K1N 6 N5
}

\section{A R T I C L E I N F O}

\section{Article history:}

Received 15 June 2011

Received in revised form

21 September 2011

Accepted 15 October 2011

Available online 20 October 2011

Keywords:

Hydrogen

Sensor

Long-range

Surface

Plasmon

Waveguide

Cytop

\begin{abstract}
A B S T R A C T
The modeling and design of membrane-supported surface plasmon polariton (SPP) waveguide devices is described for application as hydrogen $\left(\mathrm{H}_{2}\right)$ gas sensors for leak detection. The proposed structures employ thin palladium (Pd) stripes on a free-standing Cytop membrane. Various sensing architectures and design considerations are explored in detail using two-dimensional finite element method (FEM) analysis. A straight waveguide would produce a very good sensor, as a simple, compact and integrated design.
\end{abstract}

(C) 2011 Elsevier B.V. All rights reserved.

\section{Introduction}

Historically, liquid and gaseous hydrogen $\left(\mathrm{H}_{2}\right)$ has been used for applications such as rocket propellants, metallurgy and various other industrial processes. The modern global trend towards cleaner and more efficient energy sources has forged significant interest in hydrogen for fuel cells. The primary safety concern regarding the use of hydrogen is with its storage and operation. Gaseous hydrogen can be explosive at certain concentrations in air. At room temperature and atmospheric pressure this range is approximately $4 \%$ (lower explosive limit) to 74.5\% (upper explosive limit). Thus, the detection of leaks in hydrogen systems within this range becomes extremely pertinent.

Over the past decade, much advancement has occurred involving various techniques for hydrogen leak detection [1-3]. Many sensors use optical effects for detection. Optical sensors are inherently safe because electronic and optoelectronic components can be placed in a remote location from the sensor head and the latter interrogated using optical fibres which eliminates potential

\footnotetext{
* Corresponding author. Tel.: +1 6135202600.

E-mail addresses: nofong@doe.carleton.ca (N.R. Fong), berini@eecs.uottawa.ca (P. Berini), niall_tait@carleton.ca (R.N. Tait).
}

ignition via electrical sparking. Optical methods also provide high sensitivities, large dynamic ranges and immunity to electromagnetic interference $[4,5]$. There are several optical phenomena that can be exploited for gas sensing; the most prominent method is to monitor an optical change in a transduction material that selectively absorbs the analyte of interest. For hydrogen sensing, palladium (Pd) is the most prevalent material. Several sensors have been demonstrated using palladium layers and optical fibres [6,7] and fibre Bragg gratings $[8,9]$. Some of the challenges that any hydrogen sensor design must address are: sensitivity, selectivity, response time and safety.

Surface plasmon resonance (SPR) based sensors have become extremely popular in the field of chemical and biological sensing due to the high surface sensitivity of surface plasmon polaritons (SPPs). SPR is of significant interest since a small change in the refractive index of palladium upon hydrogenation can result in significant change in the propagation characteristics of the SPP. Fibre based SPR hydrogen sensors have been demonstrated previously $[10,11]$ and a sensor based on SPPs on a palladium film in the Kretschmann-Raether configuration was demonstrated long ago [12]. More recently, a modified configuration was used to obtain long-range propagation in a Pd film bounded by air on top and a finite 1D photonic crystal on a prism on the underside $[13,14]$. The assembly and interrogation set-up of prism-based structures such as these can be somewhat bulky. Other interesting structures such 


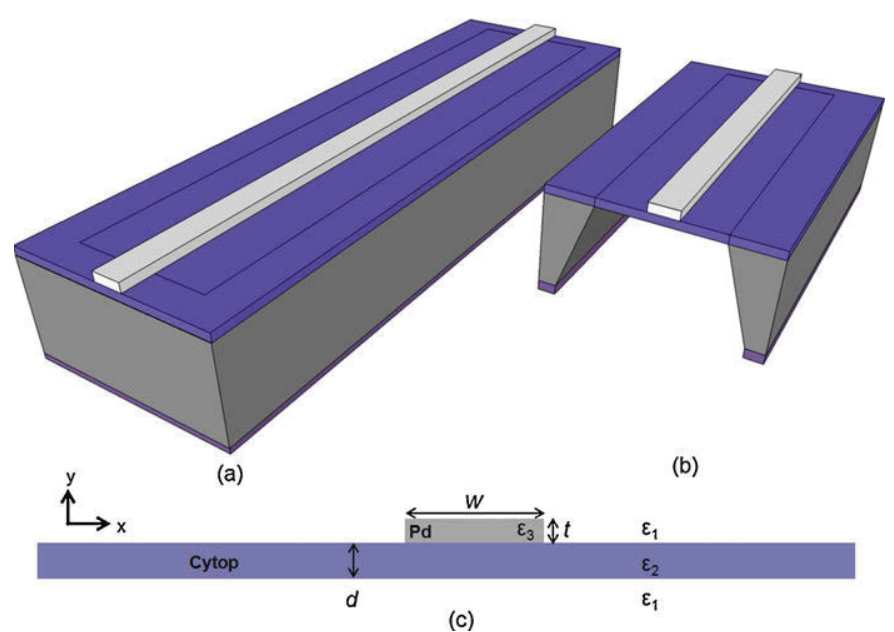

Fig. 1. (a) Example of a sensor chip featuring a Pd stripe on Cytop membrane on a silicon substrate. (b) Cut-away view showing the membrane cavity. (c) Cross sectional diagram of a membrane-supported metal stripe with labeled dimensions and permittivities.

as Pd hole arrays [15], nanoantennas [16] and nanodisks [17] have also been studied.

Long range surface plasmon polariton (LRSPP) waveguides have been demonstrated in numerous applications [18]. This integrated optic approach allows for the design of sensor chips consisting of architectures based on Mach-Zehnder interferometers and directional couplers $[19,20]$. In this paper a LRSPP palladium waveguide supported on an ultrathin, free-standing Cytop membrane is presented, with the potential to be effective as a compact, integrated hydrogen sensor. The proposed structure is sketched in Fig. 1a, with cut-away and cross-sectional views shown in Fig. 1b and c. The optical properties, sensor theory and design considerations of such structures are explored in detail in this paper.

\section{Hydrogen gas sensing with palladium}

Hydrogen is soluble in many transition metals but its solubility is particularly high in palladium which also shows high selectivity to this gas. Hydrogen absorption by such a metal proceeds as follows: $\mathrm{H}_{2}$ is first adsorbed to the Pd surface where the reaction $\mathrm{H}_{2} \rightarrow 2 \mathrm{H}$ readily occurs disassociating the gas molecules into atoms [21]. The hydrogen atoms then diffuse into the palladium forming palladium hydride $\left(\mathrm{PdH}_{x}\right.$ where $x$ represents the atomic ratio $\mathrm{H} / \mathrm{Pd}$ ). This is a reversible process [22]. The absorption of hydrogen into palladium causes several physical and optical changes in the newly formed palladium hydride such as significant permittivity change. Free electron movement in a metal can be described using the Drude model which results in the following expression for the relative permittivity:

$\varepsilon_{\mathrm{r}}=\varepsilon^{\prime}-j \varepsilon^{\prime \prime}=1-\frac{\left(\omega_{\mathrm{p}} \tau\right)^{2}}{(\omega \tau)^{2}+1}-j \frac{\omega_{\mathrm{p}}^{2} \tau}{\omega\left[(\omega \tau)^{2}+1\right]}$

where $\omega_{\mathrm{p}}$ is the plasma frequency and $\tau$ is the relaxation time given by:

$\omega_{\mathrm{p}}=\sqrt{\frac{N e^{2}}{\varepsilon_{0} m_{e}^{*}}}$

$\tau=\frac{m_{e}^{*}}{\rho N e^{2}}$

$\varepsilon_{0}$ is the permittivity of free-space, $e$ is the electronic charge, $N$ is the free electron volume density, $m_{e}^{*}$ is the effective electron mass and $\rho$ is the resistivity. From the free electron perspective, the permittivity of $\mathrm{PdH}_{x}$ changes with $x$ due to the expansion of the lattice as $x$ increases. A lattice increase results in a decrease in the free electron volume density $(N)$ which decreases the plasma frequency. The relaxation time will also decrease due to the increased density of scattering centers. These permittivity changes result in an expected net decrease in $\left|\varepsilon^{\prime}\right|$ and $\left|\varepsilon^{\prime \prime}\right|$ as $x$ increases. The permittivity of $\mathrm{PdH}_{x}$ as a function of exposure to a concentration (c) of $\mathrm{H}_{2}$ gas was modeled empirically [23] as:

$\varepsilon_{\mathrm{r}, \mathrm{PdH}_{x}}(c)=h(c) \varepsilon_{\mathrm{r}, \mathrm{Pd}}$

where $h(c)$ is a nonlinear, wavelength dependent scaling factor that is less than or equal to 1. Eq. (4) is a first order approximation for the scaling upon hydrogenation through the volumetric change to the free electron contribution in the Drude model. As the measurements in $[24,25]$ show, $h(c)$ does not scale both real and imaginary components of the permittivity equally (as Eq. (4) implies). But data from other experimentally determined permittivities for $\mathrm{PdH}_{x}$ $[26,27]$ show differences of approximately $0.01-0.05$ in the values scaling the real and imaginary parts, suggesting that Eq. (4) can be used a reasonable approximation.

The composition of palladium hydride as a function of hydrogen exposure and temperature can be described using pressurecomposition isotherms [28]. For $\mathrm{PdH}_{x}$ there are two distinct phases: $\alpha$ phase (low concentration) and $\beta$-phase (high concentration). An $\alpha$ to $\beta$-phase transition may result in an abrupt lattice expansion [21] which can lead to increased roughness and eventual delamination of the film. This transition occurs roughly at $2 \% \mathrm{H}_{2}$ at room temperature which is just below the lower explosive limit. Alloying nickel (Ni) with palladium reduces the solubility of hydrogen compared to pure palladium. Although this will effectively reduce the sensitivity of the Pd film, it should also lead to longer-term reliability and a larger dynamic range [29]. An $8 \%$ nickel alloy $\left(\mathrm{Pd}_{0.92} \mathrm{Ni}_{0.08}\right)$ film exhibits no phase transition when exposed to $100 \% \mathrm{H}_{2}$ at $1 \mathrm{~atm}$ and $300 \mathrm{~K}$, and so would be a suitable sensing film. $\mathrm{Pd}_{0.44} \mathrm{Ni}_{0.56}$ exhibits no response to hydrogen [30] and hence could be used as a reference material.

\section{Sensing architecture}

\subsection{The membrane waveguide}

The membrane waveguide design shown in Fig. 1 has been previously examined in the context of biochemical sensing [31]. Gold waveguides have been successfully fabricated on thin membranes made from silicon nitride [19] as well as Cytop [32]. The membrane waveguide can be completely immersed in the sensing environment (e.g. $\mathrm{H}_{2}$ in air) allowing the regions above and below the membrane to have matched permittivities $\left(\varepsilon_{1}\right)$. This symmetry around the waveguide is required in order to sustain LRSPP propagation [31]. The membrane must be thin and of low index such that it is not too optically invasive to prevent significant perturbations to the propagating LRSPP. For the structure under investigation here, the membrane is assumed to be made of the amorphous fluoropolymer Cytop ( $n=1.3335)$ and supported by a micromachined silicon substrate. The waveguides are assumed made of Pd and coupled to optical fibres allowing for the corresponding optoelectronics to be placed remotely if desired.

\subsection{Waveguide attenuation sensing}

A change in the imaginary part of the permittivity $\left(\varepsilon^{\prime \prime}\right)$ of the metal upon hydrogen uptake (Eqs. (1)-(4)) can be detected directly as a change in the waveguide's insertion loss. Some examples of possible optical transducer architectures for detecting changes in attenuation are shown in Fig. 2. Fig. 2a shows the simplest implementation using a straight palladium waveguide sensor. Fig. $2 \mathrm{~b}$ 
(a)

Pd

Pd

(b)

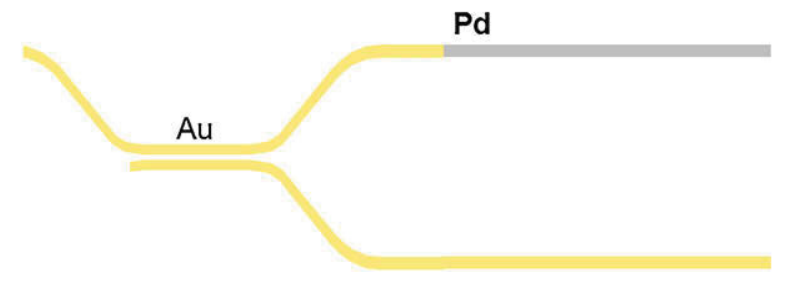

(c)

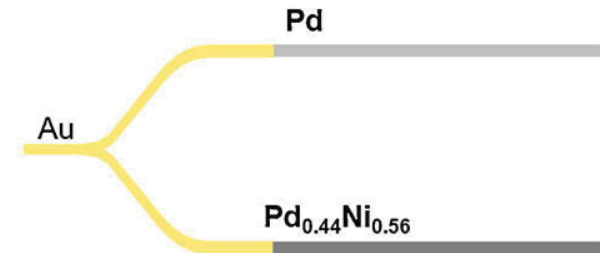

Fig. 2. Waveguide attenuation sensing architectures. (a) Straight waveguide sensor, (b) coupler sensor, (c) splitter sensor with $\mathrm{Pd}_{0.44} \mathrm{Ni}_{0.56}$ reference arm.

shows a directional coupler made with gold waveguides and a palladium sensing waveguide on one arm. The gold reference could be used to remove source fluctuations and common amplitude noise by forming the ratio of the output powers. Fig. $3 c$ is based on a splitter and uses a reference arm. This structure can be used to remove the same noise sources, but additionally can remove thermal fluctuations assuming similar thermal dependences for both arms. However the temperature must still be measured or controlled since the solubility is temperature dependent. The output power of a straight waveguide is given by:

$P_{\text {out }}=P_{\text {in }} e^{-2 \alpha L}$

where $L$ is the sensing length and $\alpha$ is the mode field attenuation coefficient in $\mathrm{m}^{-1}$. The waveguide's output power sensitivity with respect to changes in palladium hydride composition $x$, is given by:

$\frac{\partial P_{\text {out }}}{\partial x}=-2 P_{\text {in }} \overbrace{e^{-2 \alpha L} L}^{U(L)} \frac{\partial \alpha}{\partial x}$

(a)

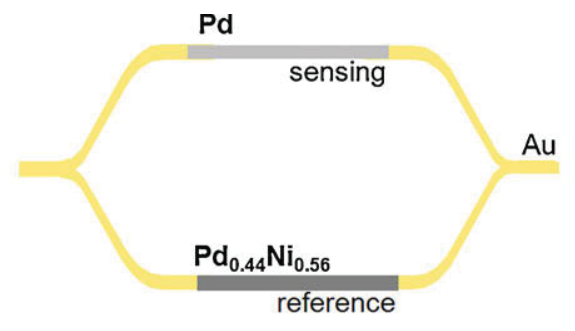

(b)

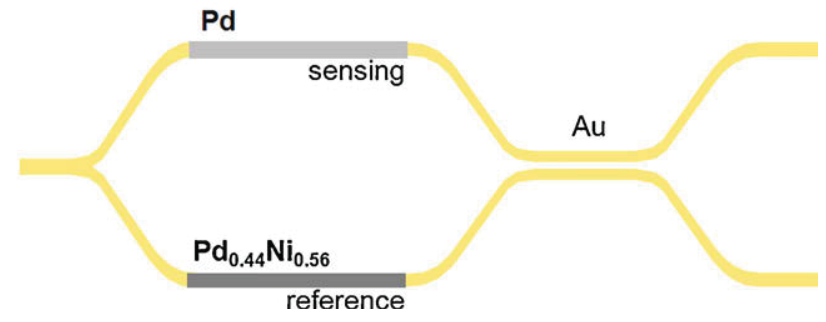

Fig. 3. Phase shift detection sensor architecture: (a) Single output MZI; (b) dual output MZI.
The sensitivity is directly proportional to the input power $\left(P_{\text {in }}\right)$. Choosing a length $L=L_{\mathrm{e}}=1 / 2 \alpha_{0}$, where $\alpha_{0}$ is the attenuation coefficient of the non-hydrogenated waveguide, maximizes the term $U(L)$ [33]. Assuming that the change in $\alpha$ is very small, which should be the case for low level leak detection, Eq. (6) becomes:

$\frac{\partial P_{\text {out }}}{\partial x}\left(L=L_{e}\right)=-e^{-1} P_{\text {in }}\left(\frac{\partial \alpha}{\partial x} / \alpha_{0}\right)$

The power sensitivity is dependent on the ratio of attenuation sensitivity to nominal attenuation: $(\partial \alpha / \partial x) / \alpha_{0}$; maximizing this ratio maximizes the power sensitivity. The attenuation may also be expressed as the mode power attenuation (MPA) where $\mathrm{MPA}=\alpha 20 \log _{10} \mathrm{e}$ in $\mathrm{dB} / \mathrm{m}$.

\subsection{Waveguide phase-shift sensing}

A change in the real part of the metal permittivity $\left(\varepsilon^{\prime}\right)$ upon hydrogen uptake causes a phase change in the LRSPP which can be monitored as a change in output power if it occurs along one arm of a Mach-Zehnder interferometer (MZI). For example, the output power of a single output MZI such as that sketched in Fig. 3a will vary as $\varepsilon^{\prime}$ of the sensing arm varies with respect to the reference arm. A dual output MZI as sketched in Fig. 3b, incorporating a $3 \mathrm{~dB}$ (50:50) coupler as the output combiner, is also envisaged, where the two (complementary) outputs can be used to remove source fluctuations and common mode noise. The output power of an equal arm single output MZI is given by:

$P_{\text {out }}=\frac{P_{\text {in }}}{2} e^{-2\left(\alpha_{\mathrm{Au}} L_{\mathrm{Au}}+\alpha L\right)}\left[1+\cos \left(\varphi_{\mathrm{D}}\right)\right]$

where $L$ is the length of the Pd sensing arm, $L_{\mathrm{Au}}$ is the optical path length of the Au splitters, coupler and access lines, $\alpha$ and $\alpha_{\text {Au }}$ are the respective mode field attenuation coefficients, and $\varphi_{\mathrm{D}}$ is the difference between the insertion phase of the sensing and reference arms, expressed as:

$\varphi_{\mathrm{D}}=\frac{2 \pi}{\lambda_{0}} L\left(n_{\mathrm{eff}, \mathrm{s}}-n_{\mathrm{eff}, \mathrm{r}}\right)$

In the above $n_{\text {eff,s }}$ and $n_{\text {eff,r }}$ are the real components of the effective indices of the modes of the sensing and reference arms respectively. For a small change in $\alpha$, the output power sensitivity to $x$ can be expressed as [33]:

$\frac{\partial P_{\text {out }}}{\partial x}=\frac{\partial P_{\text {out }}}{\partial \varphi_{\mathrm{D}}} \times \frac{\partial \varphi_{\mathrm{D}}}{\partial x}=-\frac{P_{\text {in }}}{2} e^{-2\left(\alpha_{\mathrm{Au}} L_{\mathrm{Au}}+\alpha L\right)} \sin \left(\varphi_{\mathrm{D}}\right) \frac{2 \pi}{\lambda_{0}} L \frac{\partial n_{\mathrm{eff}, \mathrm{s}}}{\partial x}$

We note from this expression that the sensitivity depends on the sensing length $L$ linearly and exponentially (i.e., same $U(L)$ as in Eq. (6)). Setting $L=L_{\mathrm{e}}=1 / 2 \alpha$ yields:

$\frac{\partial P_{\text {out }}}{\partial x\left(L=L_{e}\right)}=-\frac{P_{\text {in }}}{4} e^{-\left(1+2 \alpha_{\mathrm{Au}} L_{\mathrm{Au}}\right)} \sin \left(\varphi_{\mathrm{D}}\right)\left(\frac{\partial n_{\mathrm{eff}, \mathrm{s}}}{\partial x} / k_{\mathrm{eff}}\right)$

where $k_{\text {eff }}=\alpha / \beta_{0}$ is the normalized attenuation. From the above we note that maximizing the sensitivity requires maximizing the effective index sensitivity normalized to the normalized attenuation (a result consistent with [33]):

$\frac{\partial n_{\mathrm{eff}}}{\partial x} / k_{\mathrm{eff}}$

\section{Waveguide design space}

The geometric parameters of the waveguide are the metal width $(w)$ and thickness $(t)$, and the membrane thickness $(d)$. These must all be optimized for either maximum attenuation $(\alpha)$ sensitivity or maximum effective index ( $n_{\text {eff }}$ ) sensitivity to $x$. The relationship between the permittivity of $\mathrm{PdH}_{x}$ and its composition $(x)$ is not 


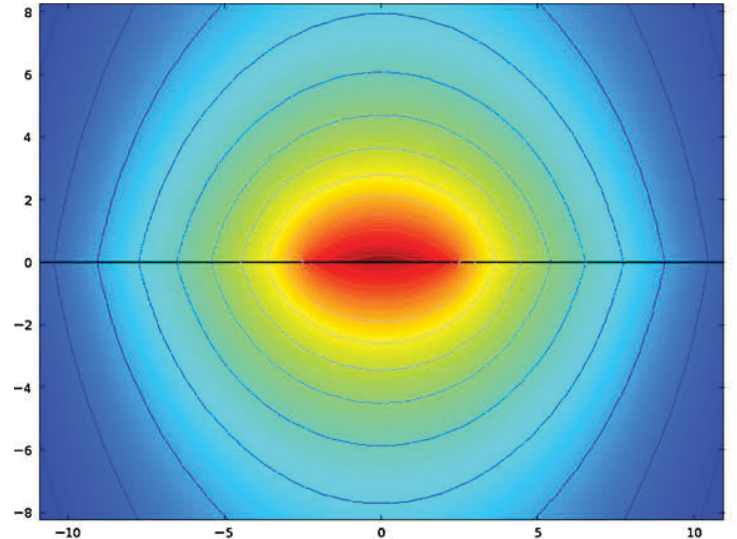

(a) t $=20 \mathrm{~nm} \quad d=10 \mathrm{~nm}$

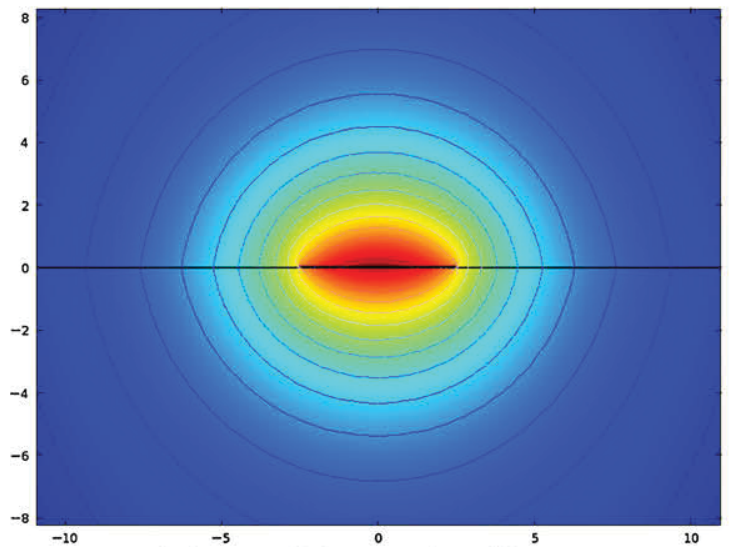

(c) $\mathrm{t}=35 \mathrm{~nm} \quad \mathrm{~d}=10 \mathrm{~nm}$

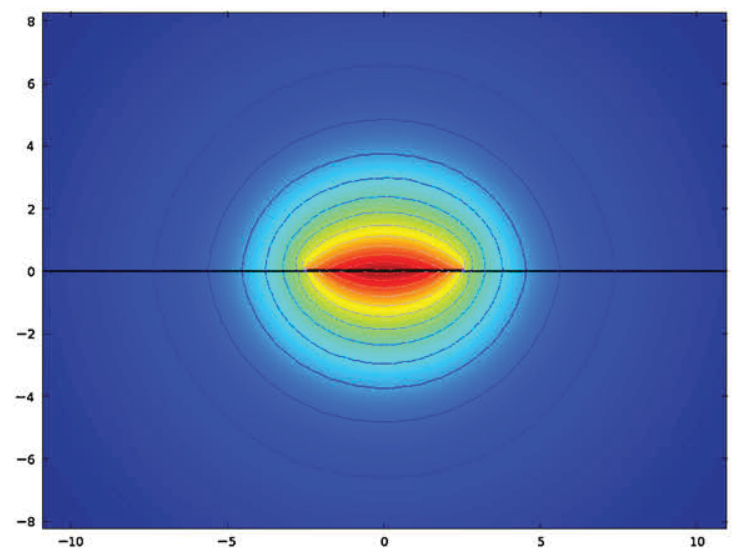

(e) $\mathrm{t}=55 \mathrm{~nm} \quad \mathrm{~d}=10 \mathrm{~nm}$

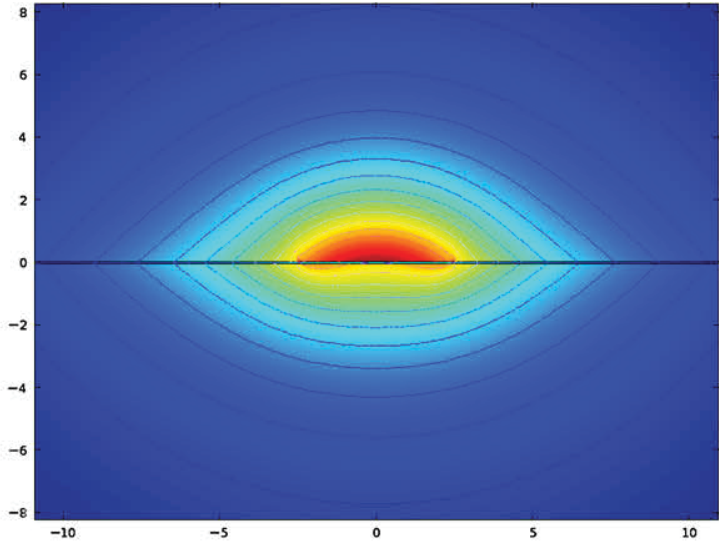

(b) $\mathrm{t}=20 \mathrm{~nm} \quad \mathrm{~d}=60 \mathrm{~nm}$

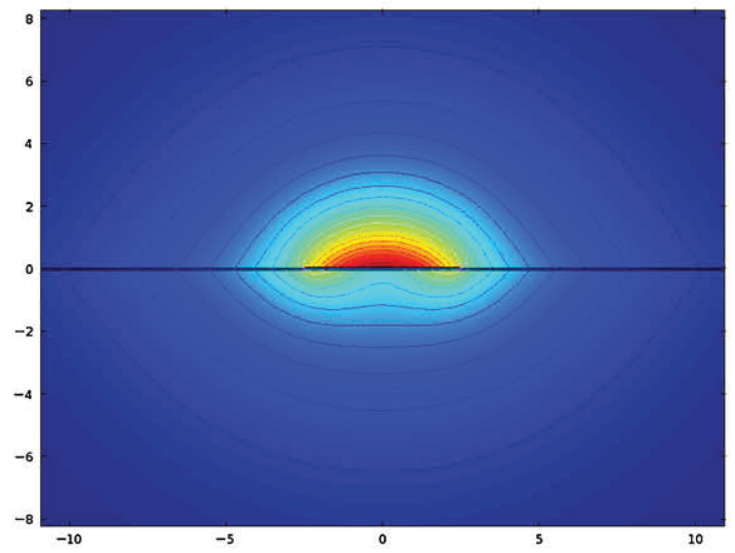

(d) $\quad t=35 \mathrm{~nm} \quad \mathrm{~d}=60 \mathrm{~nm}$

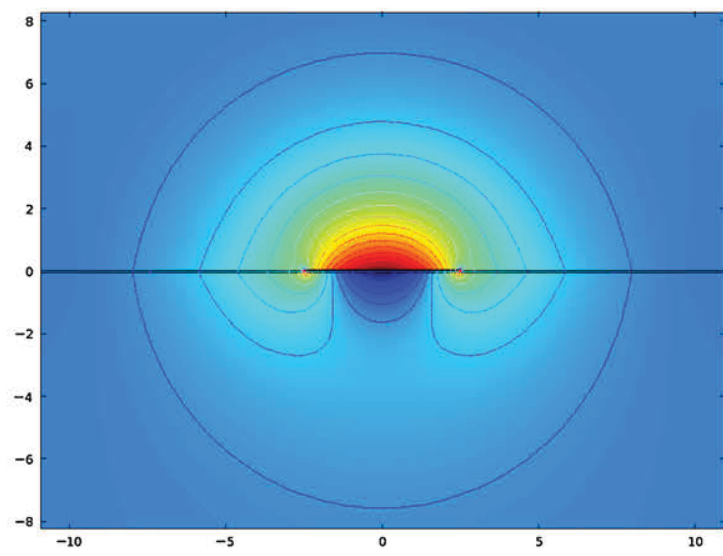

(f) $t=55 \mathrm{~nm} \quad \mathrm{~d}=60 \mathrm{~nm}$

Fig. 4. Mode field plots for the $E_{y}$ component of the $s s_{b}^{0}$ mode for a $5 \mu \mathrm{m}$ wide Pd stripe of thickness $t$ on a Cytop membrane of thickness $d$.

clearly understood so the sensitivities to $h(c)$ will be determined instead.

One dimensional mode analysis of SPPs can generally be handled analytically yielding dispersion equations that can be solved numerically. Two dimensional SPP waveguides (finite width metal as in Fig. 1c) are significantly more complex to analyze because of the lack of analytic solutions. Localization of the mode fields near the sharp metal corners results in field divergence. The finite element method (FEM) has been demonstrated to produce results of good accuracy for these structures [34]. A waveguide cross section was defined in COMSOL Multiphysics to determine the modal solutions at a free-space operating wavelength of $1550 \mathrm{~nm}$. The model consisted of a pure Pd waveguide $\left(\varepsilon_{\mathrm{r}}=-60.6764-j 49.1799\right)$ [35] on a Cytop $\left(\varepsilon_{\mathrm{r}}=1.3335^{2}\right)$ membrane in vacuum $\left(\varepsilon_{\mathrm{r}}=1\right)$.

Fig. 4 plots the $y$-component of the electric field for the LRSPP $\left(s s_{b}^{0}\right)$ mode [36] for various membrane thicknesses $(d)$ and metal thicknesses $(t)$. Each structure maintains a fixed metal width of $5 \mu \mathrm{m}$. In Fig. $4 \mathrm{a}$ we see that for a very thin metal $(t=20 \mathrm{~nm})$ and membrane $(d=10 \mathrm{~nm})$, the mode is quite symmetric. As the membrane thickness increases the mode becomes more and more 


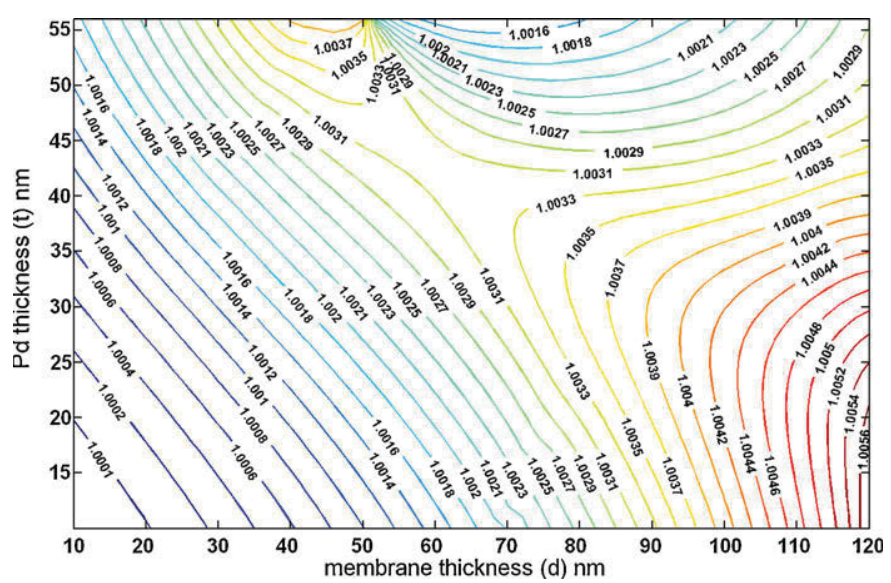

Fig. 5. Contour plot of the effective index ( $\left.n_{\text {eff }}\right)$ of the $s s_{b}^{0}$ mode for a $5 \mu \mathrm{m}$ wide Pd stripe on a Cytop membrane for ranges of $d=10-120 \mathrm{~nm}$ and $t=10-55 \mathrm{~nm}$.

perturbed by the presence of the membrane (Fig. $4 \mathrm{~b}$ ). This is due to the progression from a nearly symmetric surrounding [36] to an increasingly asymmetric one [37]. Fig. 4c and d show a similar situation but with a thicker metal $(t=35 \mathrm{~nm})$. In this situation, the combination of the thicker metal along with a thicker membrane results in a mode that is even more localized to the metal. Fig. $4 \mathrm{~d}$ and $f$ show that for a relatively thick membrane and metal the once symmetric mode profile loses symmetry about the horizontal axis. Ideally the mode should be as symmetric as possible to reduce its attenuation and improve its end-fire coupling efficiency to optical fibres. This effectively limits the suitable materials available for membranes as they need to have very low refractive indices and/or be extremely thin. Cytop was chosen for the membrane material due to its reasonably low refractive index and the fact that thin films can be fabricated.

To examine the waveguide sensitivities over a large design space, a large number of modal solutions were computed. The intention was to observe trends for the waveguide effective index and attenuation as the membrane and metal thicknesses were varied. Figs. 5 and 6 show contours for the effective index and attenuation, respectively, for $t$ and $d$ varying over the ranges $t=10-55 \mathrm{~nm}$ and $d=10-120 \mathrm{~nm}$. On both plots we see there are regions of local maxima for various designs. For thinner metals, the top (metal/air) and bottom (metal/Cytop) interfaces are coupled due to field tunneling [37]. As the waveguide and membrane become thicker these interfaces decouple and for metal thicknesses beyond about

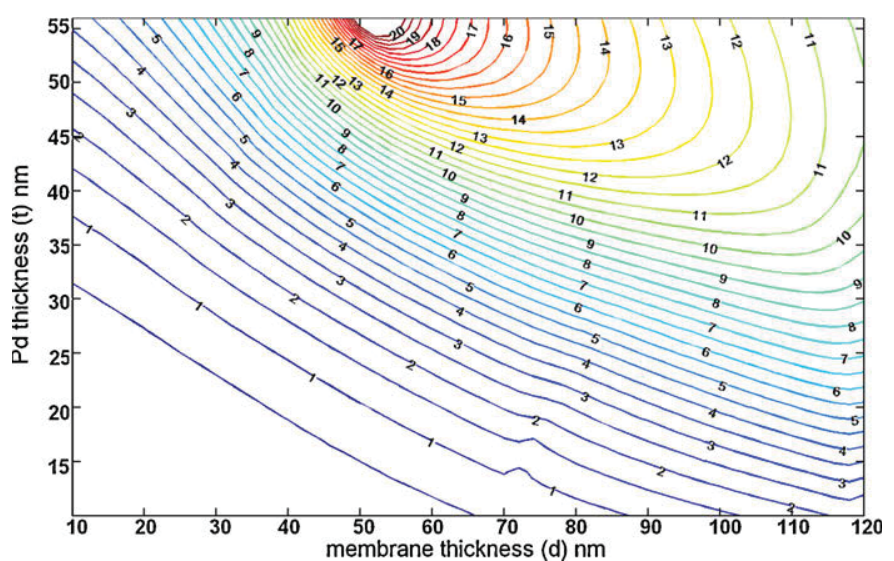

Fig. 6. Contour plot of the attenuation coefficient $\alpha\left(\mathrm{mm}^{-1}\right)$ of the $s s_{b}^{0}$ mode for a $5 \mu \mathrm{m}$ wide Pd stripe on a Cytop membrane for ranges of $d=10-120 \mathrm{~nm}$ and $t=10-55 \mathrm{~nm}$.

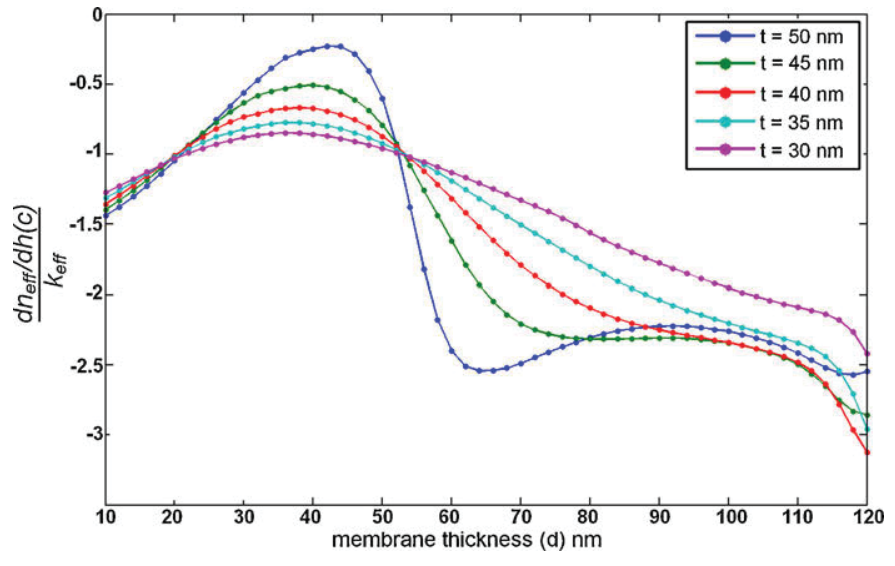

Fig. 7. Normalized sensitivity of $n_{\text {eff }}$ to $h(c)$ as a function of $d$ for specific values of $t$.

$t=55 \mathrm{~nm}$ the $s s_{b}^{0}$ mode evolves into the asymmetric profile shown in Fig. 4f. Further increasing $t$ results in a more significantly decoupled mode. At this point both the effective index and attenuation values increase rapidly.

The sensitivities (derivatives) of $n_{\text {eff }}$ and $\alpha$ were approximated using a central difference formula:

$\frac{\partial f}{\partial h} \approx \frac{f(h+\Delta h)-f(h-\Delta h)}{2 \Delta h}$

In order to determine the derivative at any point, two additional computations are needed. In order to reduce the computational effort, the sensitivities were calculated over the same range of $d$ but for specific values of $t$. The step size in Eq. (13) was taken as $\Delta h=0.01$. The normalized effective index and attenuation sensitivities are plotted in Figs. 7 and 8. From both of these plots we see that the largest normalized sensitivities occur for the thickest films. We also see that the normalized sensitivities peak at specific membrane thicknesses and then drop off. As the metal thickness increases, the proportion of the electric field exposed to the metal also increases, and thus the mode becomes more affected to changes in the metal permittivity. We also see from Fig. 4 that as the membrane thickness increases, the mode profile becomes more and more asymmetric. At certain values of $d$ the mode is localized mostly to the top surface of the structure. The membrane thickness that maximizes the exposure of the mode to the metal also maximizes the normalized sensitivity. There is however a tradeoff between sensitivity and attenuation. As more of the field is confined to the metal (by varying $d$ and/or $t$ ) the attenuation also increases due to increased absorption in the metal.

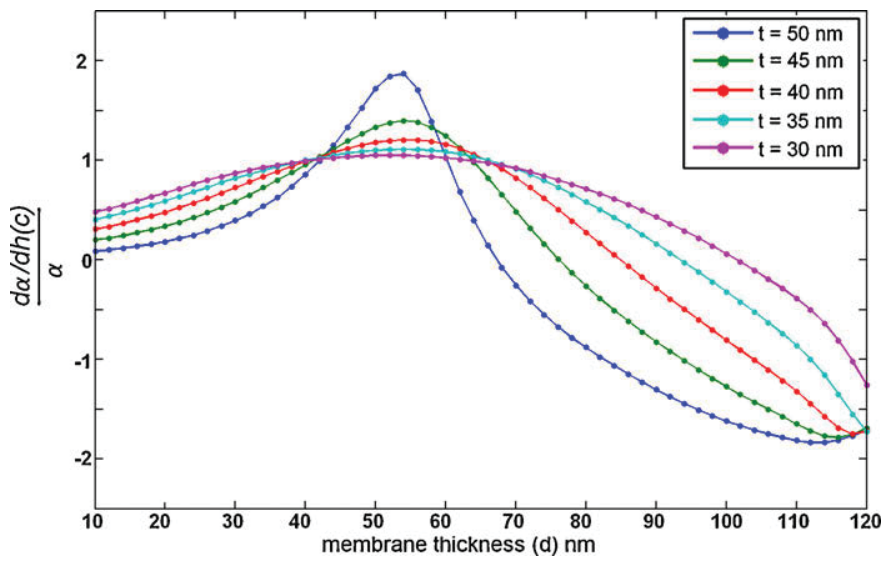

Fig. 8. Normalized sensitivity of $\alpha$ to $h(c)$ as a function of $d$ for specific values of $t$. 
Comparing Fig. 7 to Fig. 8 we note that the largest normalized sensitivities are comparable in magnitude $\left(\max \left\{\left|\left(\partial n_{\text {eff }} / \partial h(c)\right) / k_{\text {eff }}\right|\right\} \sim 2.5\right.$ and $\left.\max \{|(\partial \alpha / \partial h(c)) / \alpha|\} \sim 2\right)$. Thus, from Eqs. (7) and (11), we deduce that a straight waveguide would provide superior sensitivity compared to a MZI design. The MZI design would also be vulnerable to changes in $\alpha$ that would ultimately affect the visibility of the output.

These calculations show initial low-level detection sensitivities (i.e. pure Pd being exposed to a low concentration of hydrogen). Another point of interest is to simulate exposure to $4 \%$ hydrogen (lower explosive limit). This exposure would result in a $3.54 \%$ increase in the Pd lattice constant and a reduction of $20 \%$ in its complex refractive index [38]. Konopsky et al. observed that their structure actually showed significantly more sensitivity to a thickness increase than to a refractive index decrease [38]. Our computations for the membrane waveguide reveal a $0.04 \%$ change in $n_{\text {eff }}$ and a 33\% change in $\alpha$ for a $20 \%$ change in the complex refractive index of Pd, compared to a $0.006 \%$ change in $n_{\text {eff }}$ and $9.6 \%$ change in $\alpha$ for a 3.54\% change in Pd thickness; thus the membrane waveguide is more sensitive to a change in the refractive index of Pd than a to a commensurate change in its thickness.

The fabrication of a membrane waveguide on Cytop has previously been demonstrated [32] and such a process could be adapted to produces palladium based membrane waveguides. An integrated optical chip sensor could be excited using various methods to couple light into the waveguide. One approach would be end-fire coupling to an optical fibre but this requires trenches etched into the front surface of the wafer to accommodate the fibres and misalignments can lead to unwanted coupling into the slab waveguide formed by the air-cytop-air regions on either side of the palladium stripe. Broadside excitation could be achieved using tapered fibres [39] or integrated grating couplers [40].

Our proposed sensor may suffer similar drawbacks as any pure Pd based sensor such as cross sensitivity humidity or carbon monoxide (which could be addressed by using a Pd/Au alloy [41]). Under room temperature conditions the response time of a thin film Pd sensor is believed to be limited by surface processes [38] occurring on the order of tens of seconds.

\section{Conclusion}

The design of a LRSPP hydrogen sensor was described which has good theoretical sensitivity while providing options for compact optical integration. The devices consist of palladium stripes on ultra thin micromachined Cytop membranes. The design space was characterized and discussed with two sensor architectures presented: straight waveguides and MZIs. The structures were investigated using the 2-D finite element method. The sensor architectures envisaged depend on either maximizing the normalized sensitivity of $n_{\text {eff }}$ or maximizing the normalized sensitivity of $\alpha$ of the LRSPP with the straight waveguide designs (maximized $\alpha$ sensitivity) showing the most promising results. The modeling and design data presented provides valuable insight into the performance of the sensors proposed and highlights the principal sensor design parameters.

\section{References}

[1] F. DiMeo Jr., I. Chen, P. Chen, J. Neuner, A. Roerhl, J. Welch, MEMS-based hydrogen gas sensors, Sens. Actuators, B 117 (2006) 10-16.

[2] M.T.Soo, K.Y.Cheong, A.F.M. Noor, Advances of SiC-based MOS capacitor hydrogen sensors for harsh environment applications, Sens. Actuators, B 151 (2010) 39-55.

[3] J. Huang, W. Hsu, Y. Chen, T. Wang, H. Chen, W. Liu, Investigation of hydrogensensing characteristics of a Pd/GaN Schottky diode, IEEE Sensors J. 11 (2011) 1194-1200.

[4] T. Hüb\&TDREFS; ert, L. Boon-Brett, G. Black, U. Banach, Hydrogen sensors - A review, Sens. Actuators B 157 (2011) 329-352.
[5] M. Ando, Recent advantages in optochemical sensors for the detection of $\mathrm{H}_{2}$, $\mathrm{O}_{2}, \mathrm{O}_{3}, \mathrm{CO}, \mathrm{CO}_{2}$ and $\mathrm{H}_{2} \mathrm{O}$ in air, Trends Anal. Chem. 25 (2006) 937-947.

[6] M. Butler, Fiber optic sensor for hydrogen concentrations near the explosive limit, J. Electrochem. Soc. 138 (1991) L46-L47.

[7] J. Villatoro, D. Luna-Moreno, D. Monzon-Hernandez, Optical fiber hydrogen sensor for concentrations below the lower explosive limit, Sens. Actuators, B 110 (2005) 23-27.

[8] B. Sutapun, M. Tabib-Azar, A. Kazemi, Pd-coated elastooptic fiber optic Bragg grating sensors for multiplexed hydrogen sensing, Sens. Actuators, B 60 (1999) 27-34.

[9] C. Caucheteur, M. Debliquy, D. Lahem, P. Megret, Catalytic fiber bragg grating sensor for hydrogen leak detection in air, IEEE Photon. Technol. Lett. 20 (2008) 96-98.

[10] X. Bév\&TDREFS; enot, A. Trouillet, C. Veillas, H. Gagnaire, M. Clém\&TDREFS; ent, Surface plasmon resonance hydrogen sensor using an optical fibre*, Meas. Sci. Technol. 13 (2002) 118-124.

[11] C. Perrotton, N. Javahiraly, P. Meyrueis, M. Slaman, H. Schreuders, B. Dam, Wavelength response of a surface plasmon resonance palladium-coated optical fiber sensor for hydrogen detection, Opt. Eng. 50 (2011) 014403.

[12] B. Chadwick, M. Gal, Enhanced optical-detection of hydrogen using the excitation of surface-plasmons in palladium, Appl. Surf. Sci. 68 (1993) 135-138.

[13] V.N. Konopsky, Plasmon-polariton waves in nanofilms on one-dimensional photonic crystal surfaces, New J. Phys. 12 (2010).

[14] V.N. Konopsky, E.V. Alieva, Long-range plasmons in lossy metal films on photonic crystal surfaces, Opt. Lett. 34 (2009) 479-481.

[15] E. Maeda, S. Mikuriya, K. Endo, I. Yamada, A. Suda, J.-J. Delaunay, Optical hydrogen detection with periodic subwavelength palladium hole arrays, Appl. Phys. Lett. 95 (2009) 133504.

[16] N. Liu, M.L. Tang, M. Hentschel, H. Giessen, A.P. Alivisatos, Nanoantennaenhanced gas sensing in a single tailored nanofocus, Nat. Mater. 10 (2011) 631-636.

[17] C. Langhammer, Zorić \&TDREFS; F I., B. Kasemo, B.M. Clemens, Hydrogen storage in Pd nanodisks characterized with a novel nanoplasmonic sensing scheme, Nano Lett. 7 (2007) 3122-3127.

[18] P. Berini, Long-range surface plasmon polaritons, Adv. Opt. Photon. 1 (2009) 484-588.

[19] P. Berini, P.R. Charbonneau, N. Lahoud, Long-range surface plasmons on ultrathin membranes, Nano Lett. 7 (2007) 1376-1380.

[20] R. Charbonneau, M. Tencer, N. Lahoud, P. Berini, Demonstration of surface sensing using long-range surface plasmon waveguides on silica, Sens. Actuators B 134 (2008) 455-461.

[21] T.B. Flanagan, W.A. Oates, The palladium-hydrogen system, Annu. Rev. Mater. Sci. 21 (1991) 269-304.

[22] M.A. Butler, Optical fiber hydrogen sensor, Appl. Phys. Lett. 45 (1984) 1007-1009.

[23] X. Bevenot, A. Trouillet, C. Veillas, H. Gagnaire, M. Clement, Hydrogen leak detection using an optical fibre sensor for aerospace applications, Sens. Actuators, B 67 (2000) 57-67.

[24] K. von Rottkay, M. Rubin, P.A. Duine, Refractive index changes of Pd-coated magnesium lanthanide switchable mirrors upon hydrogen insertion, J. App. Phys. 85 (1999) 408-413.

[25] W.E. Vargas, I. Rojas, D.E. Azofeifa, N. Clark, Optical and electrical properties of hydrided palladium thin films studied by an inversion approach from transmittance measurements, Thin Solid Films 496 (2006) 189-196.

[26] Y. Yamada, K. Tajima, S. Bao, M. Okada, A. Roos, K. Yoshimura, Real time characterization of hydrogenation mechanism of palladium thin films by in situ spectroscopic ellipsometry, J. App. Phys. 106 (2009) 013523.

[27] G.K. Mor, L.K. Malhotra, D. Bhattacharyya, Effect of palladium cap layer thickness on desorption of hydrogen from PrHx films: a spectroscopic ellipsometry study, J. App. Phys. 90 (2001) 1795-1800.

[28] R.J. Wolf, M.W. Lee, R.C. Davis, Pressure-composition isotherms for palladium hydride, Phys. Rev. B 48 (1993)

[29] R.C. Hughes, W.K. Schubert, R.J. Buss, Solid-state hydrogen sensors using palladium-nickel alloys: effect of alloy composition on sensor response, J. Electrochem. Soc. 142 (1995) 249-254.

[30] R.C. Hughes, W.K. Schubert, Thin films of Pd/Ni alloys for detection of high hydrogen concentrations, J. Appl. Phys. 71 (1992) 542-544.

[31] P. Berini, R. Charbonneau, N. Lahoud, Long-range surface plasmons along. membrane-supported metal stripes, IEEE J. Sel. Top. Quantum Electron. 14 (2008) 1479-1495.

[32] N. Fong, P. Berini, R.N. Tait, Fabrication of surface plasmon waveguides on thin cytop membranes, J. Vac. Sci. Technol. A27 (2009) 614-619.

[33] P. Berini, Bulk and surface sensitivities of surface plasmon waveguides, New J. Phys. 10 (2008).

[34] P. Berini, R. Buckley, On the convergence and accuracy of numerical mode computations of surface plasmon waveguides, J. Comput. Theor. Nanosci. 6 (2009) 2040-2053.

[35] A. Borghesi, A. Piaggi, Palladium (Pd), in: E.D. Palik (Ed.), Handbook of Optical Constants of Solids II, Academic, San Diego, 1991, pp. 469-476.

[36] P. Berini, Plasmon-polariton waves guided by thin lossy metal films of finite width: bound modes of symmetric structures, Phys. Rev. B 61 (2000) 10484-10503.

[37] P. Berini, Plasmon-polariton waves guided by thin lossy metal films of finite width: bound modes of asymmetric structures, Phys. Rev. B 63 (2001) 125417.

[38] V.N. Konopsky, D.V. Basmanov, E.V. Alieva, D.I. Dolgy, E.D. Olshansky, S.K. Sekatskii, G. Dietler, Registration of long-range surface plasmon resonance by 
angle-scanning feedback and its implementation for optical hydrogen sensing, New J. Phys. 11 (2009) 063049.

[39] R. Daviau, E. Lisicka-Skrzek, R.N. Tait, P. Berini, Broadside excitation of surface plasmon waveguides on Cytop, Appl. Phys. Lett. 94 (2009).

[40] C. Chengkun, P. Berini, Grating couplers for broadside input and output coupling of long-range surface plasmons, Opt. Express 18 (2010) 8006-8018.

[41] Z. Zhao, M.A. Carpenter, H. Xia, D. Welch, All-optical hydrogen sensor based on a high alloy content palladium thin film, Sens. Actuators B: Chem. 113 (2006) 532-538.

\section{Biographies}

Norman R. Fong received the M.A.Sc. degree in Electrical Engineering and the B.Eng degree in Engineering Physics from Carleton University where he is currently working toward the Ph.D. degree in the Department of Electronics. He is part of a joint Carleton University and University of Ottawa research group that develops surface plasmon waveguides sensors. His work specifically focuses on the design, fabrication and testing of membrane-supported sensors. In 2010 he was awarded the NSERC Postgraduate Scholarship (PGS D3).

Pierre Berini received his Ph.D. and M.Sc.A. degrees in Electrical Engineering from Ecole Polytechnique de Montreal and his B.E.Sc. and B.Sc. degrees in Electrical Engineering And Computer Science respectively from the University of Western Ontario. Dr. Berini is a Professor of Electrical Engineering, Professor of Physics and University Research Chair in Surface Plasmon Photonics at the University of Ottawa.

Dr. Berini has received an NSERC E.W.R Steacie Fellowship, the University of Ottawa Young Researcher of the Year Award, a Premier of Ontario Research Excellence Award (PREA), an URSI Young Scientist Award, and is a Canada Foundation for Innovation researcher. Dr. Berini is a senior member of the IEEE and a member of the OSA. He is an Associate (Topical) Editor of Optics Express. His current research interests are focused in the area of surface plasmons and their application.

R. Niall Tait received the Ph.D. degree in electrical engineering from the University of Alberta, in 1992. He joined the Alberta Microelectronic Centre (later Micralyne) as a Research Scientist working in thin film materials and MEMS in 1992. In 1997, he joined the faculty of the Department of Electronics at Carleton University where he is currently an Associate Professor. His research interests include applying thin-film and micromachining techniques and MEMS for the development of novel components and subsystems for wireless and optical communications, sensors, and microfluidic systems. 


\section{Chapter: Design of the LRSPP Membrane Waveguide Sensor}

The following article entitled "Modeling of long range surface plasmon polariton cladded membrane waveguides with integrated grating couplers as hydrogen sensors" was published in the Journal of Applied Physics volume 117 in May 2015 and is

presented in full. The article represents work performed solely by the primary author under the supervision and guidance of the co-authors.

The sensor presented in Chapter 3 suffered from extremely large losses due to the Pd waveguide. Instead, it was proposed to use a gold waveguide with Pd over-layer as depicted figure 12(f). This implementation allowed for the Pd sensor dimensions to be independently tuned. However, this modification resulted in added asymmetry to the structure and ultimately resulted in the development of the cladded membrane waveguide from figure $12(\mathrm{~g})$ which is the topic of this chapter. Additionally, the design of broadside grating couplers is presented in order to facilitate input and output coupling. 


\title{
Modeling of long range surface plasmon polariton cladded membrane waveguides with integrated grating couplers as hydrogen sensors
}

\author{
Norman R. Fong, ${ }^{1, a)}$ Pierre Berini, ${ }^{2}$ and R. Niall Tait ${ }^{1}$ \\ ${ }^{1}$ Department of Electronics, Carleton University, Ottawa, Ontario K1S 5B6, Canada \\ ${ }^{2}$ School of Electrical Engineering and Computer Science and Department of Physics, University of Ottawa, \\ Ottawa, Ontario K1N 6N5, Canada
}

(Received 10 March 2015; accepted 23 April 2015; published online 30 April 2015)

\begin{abstract}
The design of a long range surface plasmon polariton cladded membrane waveguide with grating couplers is proposed. The device consists of a gold stripe embedded in a thin Cytop membrane with a palladium over-layer and can be used as a hydrogen sensor. Input and output light coupling is achieved through integrated gold grating couplers directly on the waveguides. The design is approached through finite element method modeling. Waveguide and sensor designs are compared and discussed via a 2D modal analysis. The design and optimization of input and output grating couplers are also presented. @ 2015 AIP Publishing LLC. [http://dx.doi.org/10.1063/1.4919758]
\end{abstract}

\section{INTRODUCTION}

Hydrogen gas $\left(\mathrm{H}_{2}\right)$ as a fuel source has garnered significant interest due to its ability to produce emissions-free power through implementation as fuel cells. ${ }^{1}$ The recent proliferation of commercial fuel cells by the automotive industry has produced increased interest in smaller scale applications. However, hydrogen gas is inherently dangerous as it is combustible for concentrations in the range of $4 \%$ (lower explosive limit) to $74.5 \%$ (upper explosive limit) in air. Therefore, the need for compact, fast, safe, and accurate detection of leaks in a hydrogen system is a safety necessity.

Numerous techniques have been implemented for hydrogen sensing ${ }^{2}$ however, optical devices generally provide the safest options due to the lack of any heating or electrical components which could potentially ignite the atmosphere. There are several implementations that can be used for optical hydrogen sensing such as optical fibers, ${ }^{3,4}$ fiber Bragg gratings, ${ }^{5,6}$ and surface plasmon resonance (SPR) structures. $^{7-9}$ This report builds upon the previous work exploring the use of Long Range Surface Plasmon Polaritons (LRSPPs) ${ }^{10}$ which have the potential for very accurate and sensitive detection due to their inherently high surface sensitivity and their long optical interaction length with the sensing medium.

SPPs are TM-polarized charge-density surface waves that propagate along the interface between a semi-infinite metal and a semi-infinite dielectric at optical wavelengths. ${ }^{11,12}$ In this situation, the wave is referred to as a single-interface SPP and is highly lossy due to strong confinement to the metal. Losses can be reduced by having a thin metal film or stripe symmetrically bounded by a uniform dielectric. Such a structure can propagate a low-loss LRSPP mode. ${ }^{13}$

This article reports the optical modeling and design of a LRSPP waveguide that can be used as a hydrogen sensor. The structure is based on an thin Au stripe embedded in a thin dielectric free-standing membrane with a Pd patch as

\footnotetext{
a) Author to whom correspondence should be addressed. Electronic mail: nofong@doe.carleton.ca
}

the $\mathrm{H}_{2}$ sensing medium and grating couplers to in- and out-couple incident TM-polarized light. Designs are analyzed through finite element method (FEM) modeling.

\section{STRUCTURE AND OPERATION}

\section{A. Device structure}

The sensor structure diagrammed in Fig. 1 is referred to as the cladded membrane waveguide and consists of a gold $\mathrm{Au}$ ) stripe embedded in a free-standing dielectric membrane. Light coupling is achieved by integrating grating couplers directly on the waveguides. The devices are designed to work at an operating wavelength of $1550 \mathrm{~nm}$. Most hydrogen sensors utilize palladium (Pd) as it readily forms palladium hydride proportional to hydrogen exposure levels. Our device uses a thin palladium over-layer for transduction.

\section{B. Cladded membrane waveguide operation}

LRSPPs have previously been explored in detail. ${ }^{13-15} \mathrm{~A}$ main requirement for LRSPP propagation is that the metal

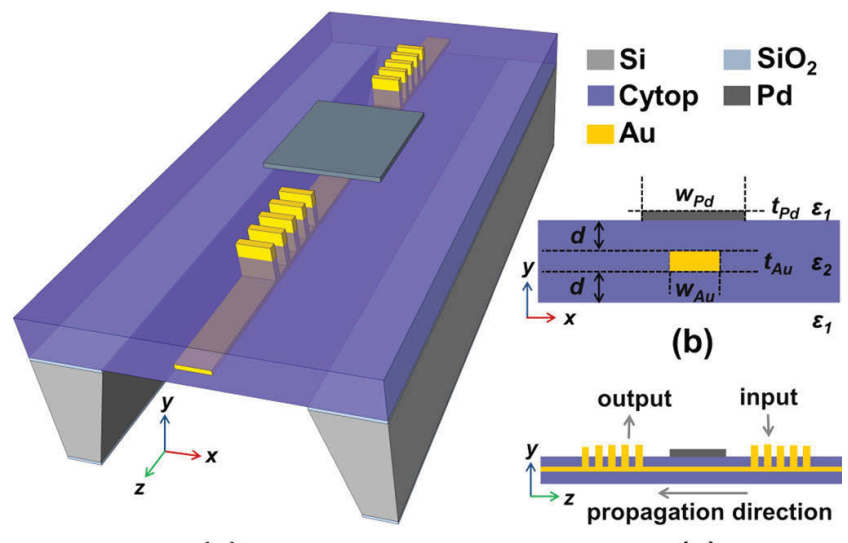

(a)

(c)

FIG. 1. (a) 3D sketch of a cladded membrane waveguide sensor with embedded grating couplers (dimensions not to scale). (b) Front cross-section through the sensor and (c) side cross-section through the sensor. 
stripe be surrounded symmetrically by dielectric. For any sensing application, the waveguide mode fields need to interact with the sensing environment and thus full encasement is impractical. It has been shown that a thin, optically nonintrusive membrane can be used to suspend the stripe while allowing the environment to symmetrically form the cladding. This is referred to as the membrane waveguide. ${ }^{10,16}$ The cladded membrane waveguide takes a similar but modified approach by embedding the stripe into the membrane, resulting in increased symmetry over those previous designs.

The stripe is symmetrically surrounded above and below by a finite thickness dielectric (Cytop) with thickness $d$, permittivity $\varepsilon_{2}$, and placed in a gaseous environment of permittivity $\varepsilon_{1}$ as depicted in Fig. 1(b). The environment and thin membrane form the refractive index symmetry required to sustain the LRSPP. A region of this structure can be functionalized to be hydrogen sensitive by depositing a thin layer of palladium over the waveguide as depicted.

A polarization maintaining single mode fiber is assumed to excite the LRSPP mode via the input grating. The LRSPP will then propagate along the waveguide and sensor to scatter into output light from the output grating as depicted in Fig. 1(c). The output light could be captured using, for example, a multimode optical fiber. Exposure to hydrogen will change the permittivity of the Pd patch resulting in a measureable change of the output power from the output grating.

\section{Device materials}

The membrane dielectric is Cytop, which is a chemically resistant amorphous fluoropolymer with low refractive index $(\sim 1.335)$ that has previously been demonstrated in other LRSPP devices and sensors. ${ }^{17-19}$ This low refractive index allows for a fairly thick and mechanically stable structure while still being optically non-invasive. Gold is used as the waveguide material due to its relatively long propagation lengths at the desired wavelength. Although other hydrogen absorbing material options are available (i.e., palladium/ nickel alloy, ${ }^{20}$ palladium/gold alloy, ${ }^{21}$ and palladium/silver alloy $^{22}$ ), pure Pd was used for simplicity. Palladium is very lossy at optical wavelengths and therefore the patches need to be kept relatively small to ensure a detectable output signal level.

The substrate consists of a silicon wafer with silicon dioxide layers which are used as the back mask and etch stop. The substrate is for structural purposes and is far away from the optical path such that it does not interact. The structure and fabrication steps have been previously presented. ${ }^{23}$

\section{Grating couplers}

Of the various methods for exciting surface plasmons in planar waveguides, the simplest is end-fire excitation, which can provide near $100 \%$ efficiency. ${ }^{24-26}$ This method requires a precisely flat end facet which may be difficult to achieve, especially for the case of a free-standing membrane. Tapered fibers $^{27}$ have also been demonstrated, but they require extremely delicate alignment. Directly integrated grating couplers allow for broadside (perpendicular to the waveguide) single mode excitation and optical output with flat optical fibers. Although coupling efficiency will be sacrificed compared to other methods, broadside coupling allows for relatively simpler alignment and has fewer mechanical limitations.

\section{DEVICE MODELING}

\section{A. Hydrogen sensing}

Hydrogen readily absorbs into various transition metals but has a particularly high solubility, selectivity, and partial reversibility with palladium. ${ }^{28} \mathrm{H}_{2}$ uptake in $\mathrm{Pd}$ forms palladium hydride $\left(\mathrm{PdH}_{\mathrm{x}}\right.$, where $x$ is the atomic ratio of $\mathrm{H}$ to $\left.\mathrm{Pd}\right)$ proportional the atmospheric $\mathrm{H}_{2}$ concentration $(c)$. The complex permittivity of the palladium hydride has been previously modeled as

$$
\varepsilon_{r, P d H_{x}}=h(c) \cdot \varepsilon_{r, P d},
$$

where $h(c)$ is a non-linear concentration-dependent scaling factor. ${ }^{10,29,30}$ This direct scaling of the permittivity is known to be a fairly crude model of $\mathrm{PdH}_{\mathrm{x}}$ formation but the simplicity of this model allows for the ability to examine sensitivity as a function of design parameters.

\section{B. Waveguide modal analysis}

The waveguide and sensing properties can generally be determined through 2D modal analyses of the structure cross-sections using the finite element method (e.g., COMSOL Multiphysics). The metals were treated as lossy dielectrics with relative permittivity of $\varepsilon_{r, P d}=-60.6764$ $-49.1799 \mathrm{i}$ and $\varepsilon_{r, A u}=-131.9475-12.857 \mathrm{i}$ at the operating wavelength $^{31}$ of $1550 \mathrm{~nm}$. The membrane was modeled as an ideal dielectric using $\varepsilon_{r, \text { Cytop }}=1.78$ for Cytop and the sensing environment was taken as air $\left(\varepsilon_{r, l}=1\right)$.

There are several design parameters that can be independently explored in this structure. The most influential dimensions are the membrane/cladding thickness $(d)$ and the thickness of each metal layer $\left(t_{A u}\right.$ and $\left.t_{P d}\right)$. The width of the Au stripe $\left(w_{A u}\right)$ and the palladium patch $\left(w_{P d}\right)$ generally have a less significant effect so a typical Au stripe width of $5 \mu \mathrm{m}$ was used while the palladium patch was made $10 \mu \mathrm{m}$ wide to simplify alignment during fabrication.

A practical parameter for analysis is the mode power attenuation (MPA) ${ }^{32}$ which represents the loss per length of the waveguide and is defined as

$$
\operatorname{MPA}=\alpha \cdot 20 \cdot \log (\mathrm{e}),
$$

where $\alpha$ is the field attenuation coefficient of the mode. Fig. 2 shows the computed mode profiles (y-component of the electric field) and calculated MPA for a sample waveguide without palladium contrasted with the same structure with a thin Pd film $\left(t_{P d}=5 \mathrm{~nm}\right)$. The waveguide portion has a symmetric mode profile along with a relatively low MPA, but the addition of a thin palladium layer dramatically increases the MPA by multiple orders of magnitude and induces a slightly asymmetric profile.

Precise fabrication control is required in order to obtain the expected performance. As an example, a 10\% increase in 


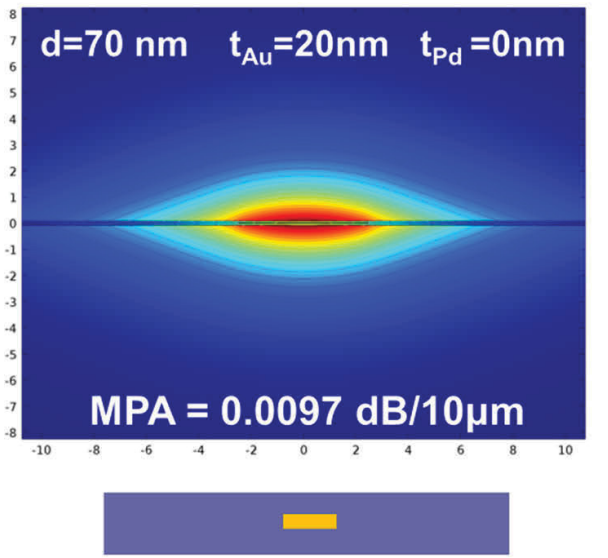

(a)

the thicknesses of the Au-only waveguide would result in an order of magnitude increase in the MPA. Although such an increase is undesirable, it is not critical.

\section{Membrane thickness analysis}

A practical limitation is that the membrane needs to be thick enough such that it can be realistically fabricated and mechanically stable. Fig. 3 shows the mode profile for various membrane thicknesses with fixed $t_{A u}=20 \mathrm{~nm}$ and $\mathrm{t}_{\mathrm{Pd}}=5 \mathrm{~nm}$. As the membrane thickness is increased, the separation between the two metals also increases which results in an increasingly asymmetric structure. As this separation increases the electric field confinement begins to favor the
$\mathrm{Pd} /$ Cytop interface over the $\mathrm{Au}$ stripe which results in an increased mode attenuation. Recall that the parameter $d$ is the thickness of the membrane halves about the Au stripe, so the thickness of the total membrane stack is $2 d+t_{A u}$ as depicted in Fig. 1(b).

Previous work examined the mechanical properties of Cytop $^{33}$ and the fabrication of Cytop membranes. ${ }^{23,34}$ From the perspective of minimizing losses, the thinnest possible membranes would be desirable. However, it was very difficult to fabricate membranes with $\mathrm{d} \approx 30 \mathrm{~nm}$ with any consistent yield. ${ }^{23}$ Therefore, a design thickness of $d=70 \mathrm{~nm}$ was chosen as a reasonable compromise between mechanical stability and loss. With this design, a $100 \mu \mathrm{m}$ long Pd patch could be used such that a sensor with an overall
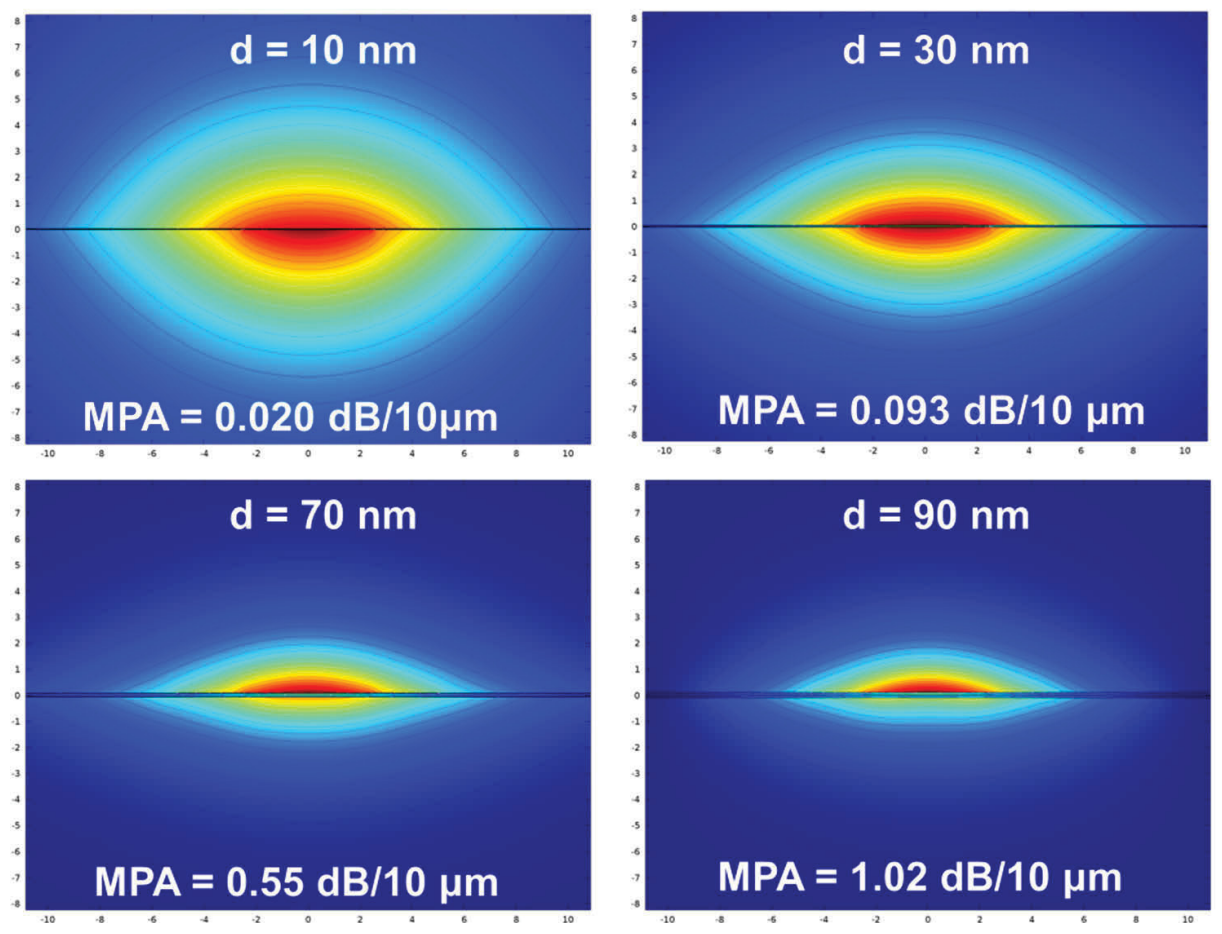

FIG. 3. Mode profiles and computed MPA for various membrane thicknesses $(\mathrm{d}=10 \mathrm{~nm}$ to $90 \mathrm{~nm})$ with $\mathrm{t}_{\mathrm{Au}}=20 \mathrm{~nm}$ and $\mathrm{t}_{\mathrm{Pd}}=5 \mathrm{~nm}$. 
acceptable propagation loss of about $5.5 \mathrm{~dB}$ would be achieved.

One potential drawback of using relatively thick membranes is that there may be leakage through the slab modes supported by the air/Cytop/air slab waveguides on either side of the metal stripe. Fig. 4 shows a comparison of the computed mode effective index $\left(n_{\text {eff }}\right)$ for the cladded Au stripe waveguide compared to the effective index of the $\mathrm{TM}_{0}$ mode supported by the air/Cytop/air slab waveguide of equal thickness. It is shown that for all membrane designs in question, the effective index of the Au stripe membrane waveguide is higher than that of the air/Cytop/air slab waveguide and thus leakage to the slab will not occur.

\section{Hydrogen sensitivity}

The design space over gold and palladium thicknesses was computed under the established limitation of $d=70 \mathrm{~nm}$. Fig. 5(a) shows a plot of the normalized sensitivity of $\alpha$ to $h(c)$ which is computed using a central-difference formula approximation ${ }^{10}$ with a step size of $\Delta h(c)=0.01$. Fig. 5(b) shows a plot of the MPA for each of the designs.

There is a tradeoff between attenuation and sensitivity, however, there is no particular need to minimize passive attenuation as the MPA is within acceptable limits. Similarly, there is no necessity to maximize sensitivity as even the nonoptimal designs still have very high computed relative sensitivities. Therefore, a moderate compromise design was chosen with $\mathrm{d}=70 \mathrm{~nm}, \mathrm{t}_{\mathrm{Au}}=20 \mathrm{~nm}$, and $\mathrm{t}_{\mathrm{Pd}}=5 \mathrm{~nm}$.

The behavior of $h(c)$ is not particularly well-known so any estimate of a detection limit or sensitivity is highly approximate. However, a rough estimate can be approached by making a few assumptions. We assume a conservative detection resolution limit of $0.01 \mathrm{~dB}$ (factoring in detector precision, background noise and stability, etc.) and a $100 \mu \mathrm{m}$ long Pd sensor patch. The design sensitivity derived in Fig. 5(a) would correspond to an approximate $h(c)$ change of $4.4 \times 10^{-5}$ for the defined resolution. It has been previously modeled that upon exposure to $4 \%$ hydrogen, a $20 \%$

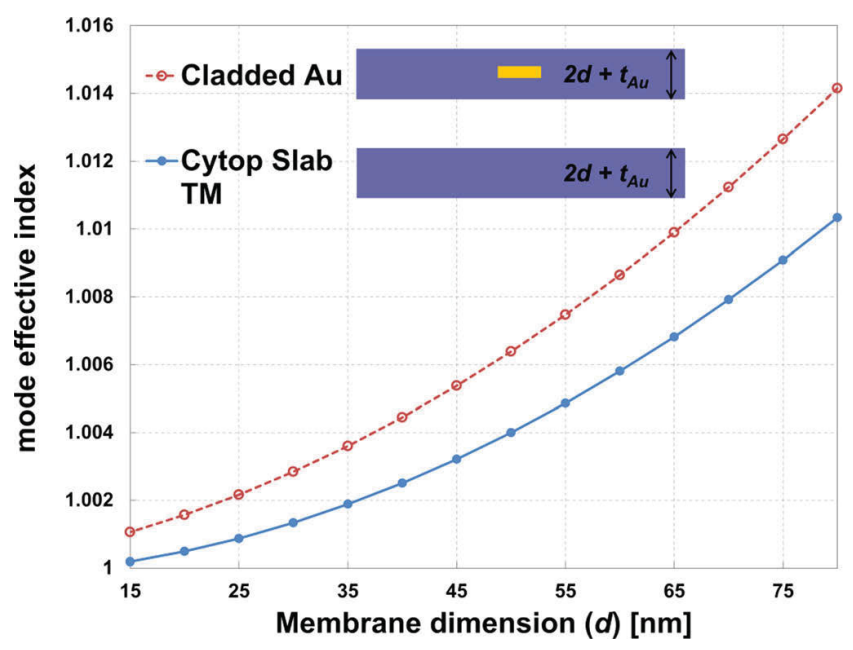

FIG. 4. Plot comparing the effective index of the Au stripe cladded membrane waveguide to that of the $\mathrm{TM}_{0}$ mode supported by the air/Cytop/air slab waveguide.

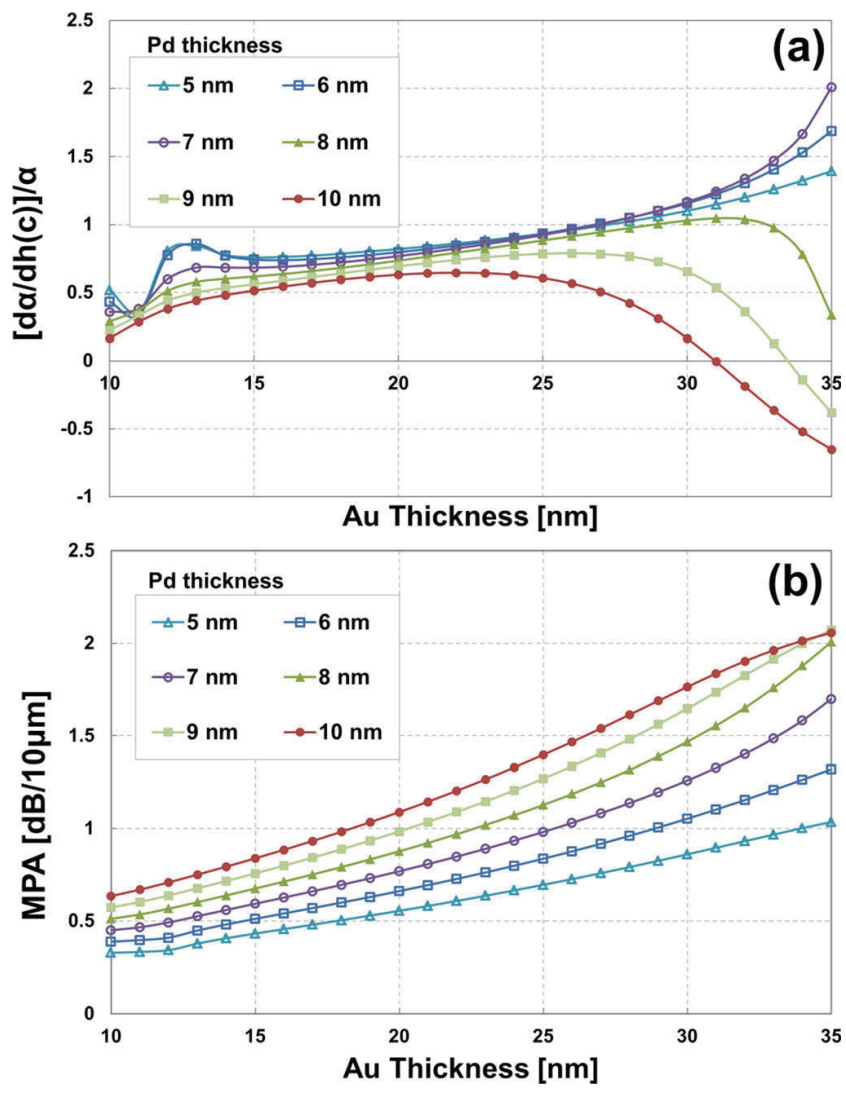

FIG. 5. Design space plots of varying gold and palladium thicknesses computed through FEM. (a) Normalized mode attenuation coefficient sensitivity and (b) mode power attenuation.

reduction in the refractive index of palladium was observed. ${ }^{35}$ Using a crude linear estimate, this would result in a resolution of approximately $2.8 \times 10^{-4} \%$ or $\sim 2.8 \mathrm{ppm}$. This rough approximation is significantly lower than the minimum required limit of $0.1 \%$ as defined by the National Renewable Energy Laboratory (NREL) and Department of Energy (DOE) ${ }^{36}$ Ultimately, the sensitivity will depend on the resolution and stability of the experimental setup.

\section{GRATING COUPLER DESIGN}

\section{A. Grating coupler parameters}

For simplicity, the grating couplers of Fig. 1(a) are modelled in 2D following the cross-section sketched in Fig. 1(c). An input grating coupler is diagrammed in Fig. 6 with the three important design parameters being: bump height $(H)$, bump width (w), and pitch $(\Lambda)$. The coupling will also be strongly depending on the input beam height $(s)$ and input beam position $(p)$. The leftmost grating edge is defined at position $z=0$ with the intention to maximize power propagation in the $-z$ direction. The domain boundaries are treated as perfectly matched layers (PMLs) to eliminate reflections.

The input is a z-axis polarized 1D Gaussian beam with a mode field diameter $\left(1 / e^{2}\right.$ intensity) approximated as $8 \mu \mathrm{m}$. Fig. 7 shows a truncated portion of the computed $E_{y}$ field for a large simulation domain used to compute the steady state solution. The grating also excites several higher order and radiative modes. These will quickly attenuate or radiate 


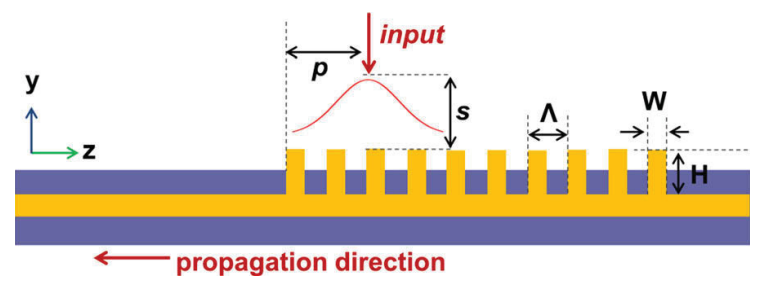

FIG. 6. Cross section of the input grating coupler with design dimensions labelled. Dimensions are not depicted to scale.

away and only the LRSPP mode will persist. The insets in Fig. 7 show $\mathrm{E}_{\mathrm{y}}$ cross sections at various $z$ locations from the grating. At approximately $z=-100 \mu \mathrm{m}$, the mode has stabilized to the expected symmetric profile.

The overall performance depends on both the grating design and the input fiber position which are independently explored in Secs. IV B and IV C. Although they are presented in a linear fashion, the design is actually an iterative process where an arbitrary input position was used to determine a temporary grating design, which is then used to re-optimize the input position, which is then used to re-optimize the grating design again. For simplicity, these sections show only the final iterations.

\section{B. Input grating dimensions}

Competing grating designs are evaluated by comparing the total power coupled to the LRSPP mode $P(z)$ at a position $\mathrm{z}$, far from the grating. This was determined by integrating the $z$-directed time-averaged power flow (Poynting vector) across a vertical section at $z=-100 \mu \mathrm{m}$. Initially, an approximate value for the grating period $(\Lambda)$ was obtained from the phase matching condition ${ }^{37}$ given by the following equation:

$$
\Lambda \approx \lambda_{0} / \mathrm{n}_{\mathrm{eff}, \mathrm{LRSPP}}
$$

where $n_{\text {eff,LRSPP }}$ is the effective index of LRSPP mode. The cladded membrane waveguide without palladium has an effective mode index of $n_{\text {eff,LRSPP }} \approx 1.013$; therefore, for $\lambda_{0}=1550 \mathrm{~nm}$, a starting value of $\Lambda=1530 \mathrm{~nm}$ was chosen and a design domain of various bump widths and heights was simulated with the input position defined as described in Sec. IV C. From these results, a good design was selected and used to refine the value of the pitch. Fig. 8 shows the

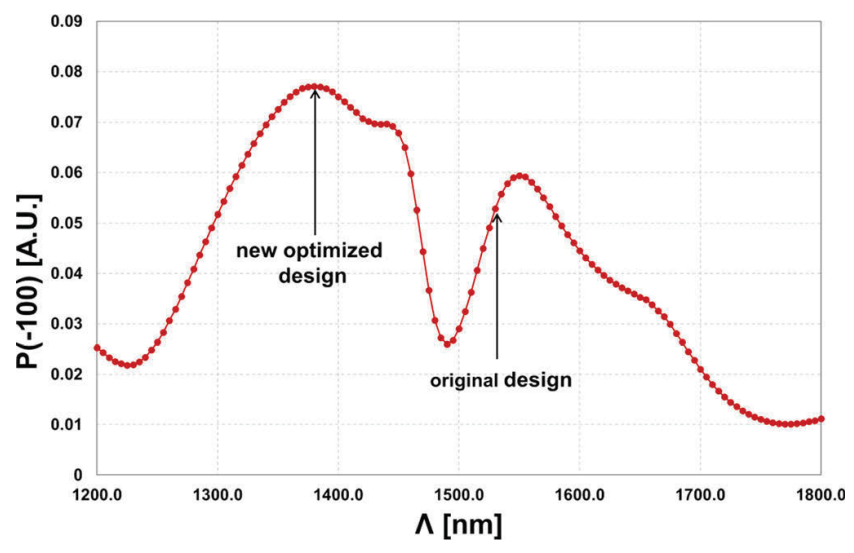

FIG. 8. Plot of the propagated power far from the grating as a function of grating pitch using a temporary design.

simulated $P(-100)$ as a function of the grating pitch using the temporary bump height and width. The largest power coupling is achieved at $\Lambda=1380 \mathrm{~nm}$. This is noticeably different than the initial guess, however. Equation (3) is generally only accurate for weakly perturbed gratings, which is not the case with this design.

Using this new pitch, the bump design space was recomputed with the $P(-100)$ results shown in Fig. 9. A clear maximum appears at $\mathrm{H}=225 \mathrm{~nm}$ and $\mathrm{w}=740 \mathrm{~nm}$ for $\Lambda=1380 \mathrm{~nm}$.

\section{Input beam position}

For simplicity, only perpendicular incidence was considered, thus the position of the input beam can be defined by the height from the grating $(s)$ and the beam center position $(p)$. The input height was fixed to $s=2 \mu \mathrm{m}$. Fig. 10 shows the LRSPP mode power propagating along $\pm z$ at $\sim 100 \mu \mathrm{m}$ from each side of the grating as a function of various input positions $p$. As expected, when the input is centered on the grating, equal power is coupled along both $\pm z$ directions. Offsetting the input towards the desired propagation direction will result in $\sim 5 \times$ larger coupling with the maximum position for the $-z$ direction at $z=+1 \mu \mathrm{m}$.

\section{Coupling efficiency}

The previous analysis used the absolute mode power at a distance far from the grating to determine an optimal

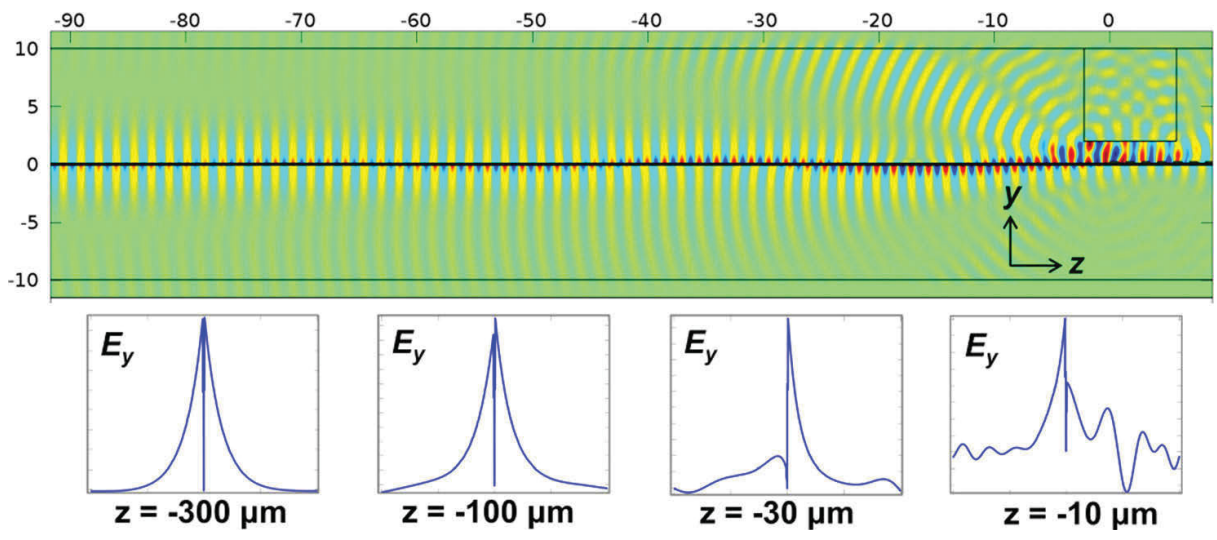

FIG. 7. A portion of the long simulation domain used to model the input grating coupler with LRSPP propagating in the $-\mathrm{z}$ direction. Mode profile cross sections are presented at various distances from the grating. 


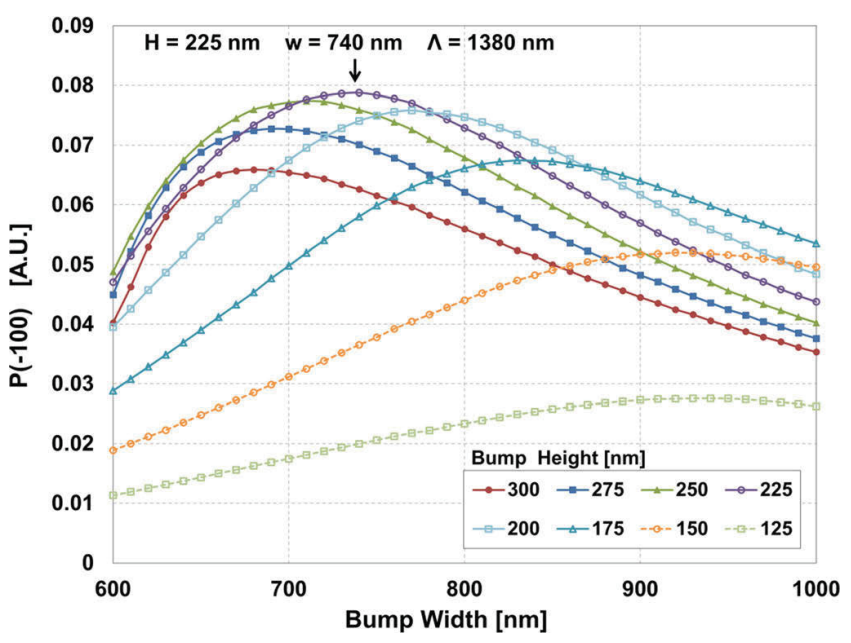

FIG. 9. Plot of optimized grating design (bump widths and heights) using for $\Lambda=1380 \mathrm{~nm}$.

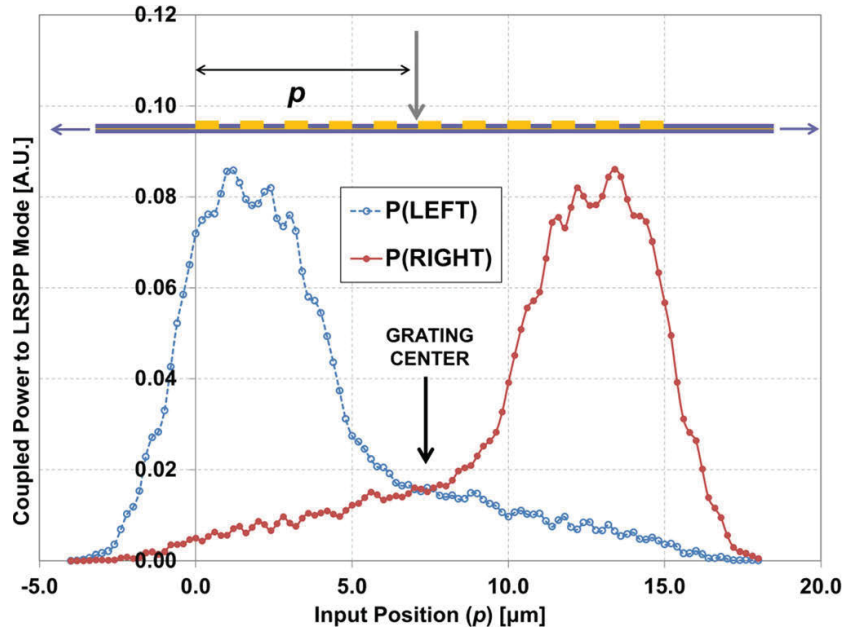

FIG. 10. Plot of the propagated power far from each side of the grating as a function of input position $p ; p=0$ represents the left edge of the grating. design. To isolate and calculate the grating coupling efficiency, additional analysis is required. The total power propagating along the $-z$ direction can be expressed as ${ }^{38}$

$$
P(z)=P_{0} e^{2 \alpha z}+P_{r a d}(z)
$$

where $P_{O}$ is the power carried by the LRSPP mode directly at the grating edge $(z=0), \alpha$ is the attenuation constant of the LRSPP, and $P_{\text {rad }}(z)$ is the power carried by all other modes, which will decay rapidly. At positions far from the grating, Eq. (4) can be approximated by the first term only. If $P_{O}$ is normalized by the input beam power, then as $z \rightarrow-\infty, P_{0}$ will directly represent the input coupling efficiency.

A long computational domain using the optimum grating design and beam position was used and $P(z)$ extracted at various positions $z$ from which the normalized $P_{O}$ was calculated. Fig. 11 shows a plot of the normalized $P_{0}$ versus $|1 / z|$. Using a smoothing spline numerical curve fit, the coupling efficiency was extrapolated as the y-intercept and determined to be 0.1901 corresponding to a coupling loss of $7.2 \mathrm{~dB}$.

\section{E. Output coupling}

For simplicity, the output grating design is the same as the input grating. This allows the devices to be used in either direction, although the output coupling efficiency may not necessarily be optimal in this case. Output coupling can be analyzed in a similar manner as the input, but by comparing the $+y$-directed power flow as shown in Fig. 12(a) to the $-z$ directed flow at the edge of the grating as in Fig. 12(b), when the LRSPP is launched along the waveguide toward the grating. The total output power is integrated over a $50 \mu \mathrm{m}$ wide window to represent the core diameter of a multimode fiber.

The output grating edge will reflect some power and excite other modes. To remove any back-reflected power, the mode power is measured at various distances away from the grating and extrapolated forward again using Eq. (4).

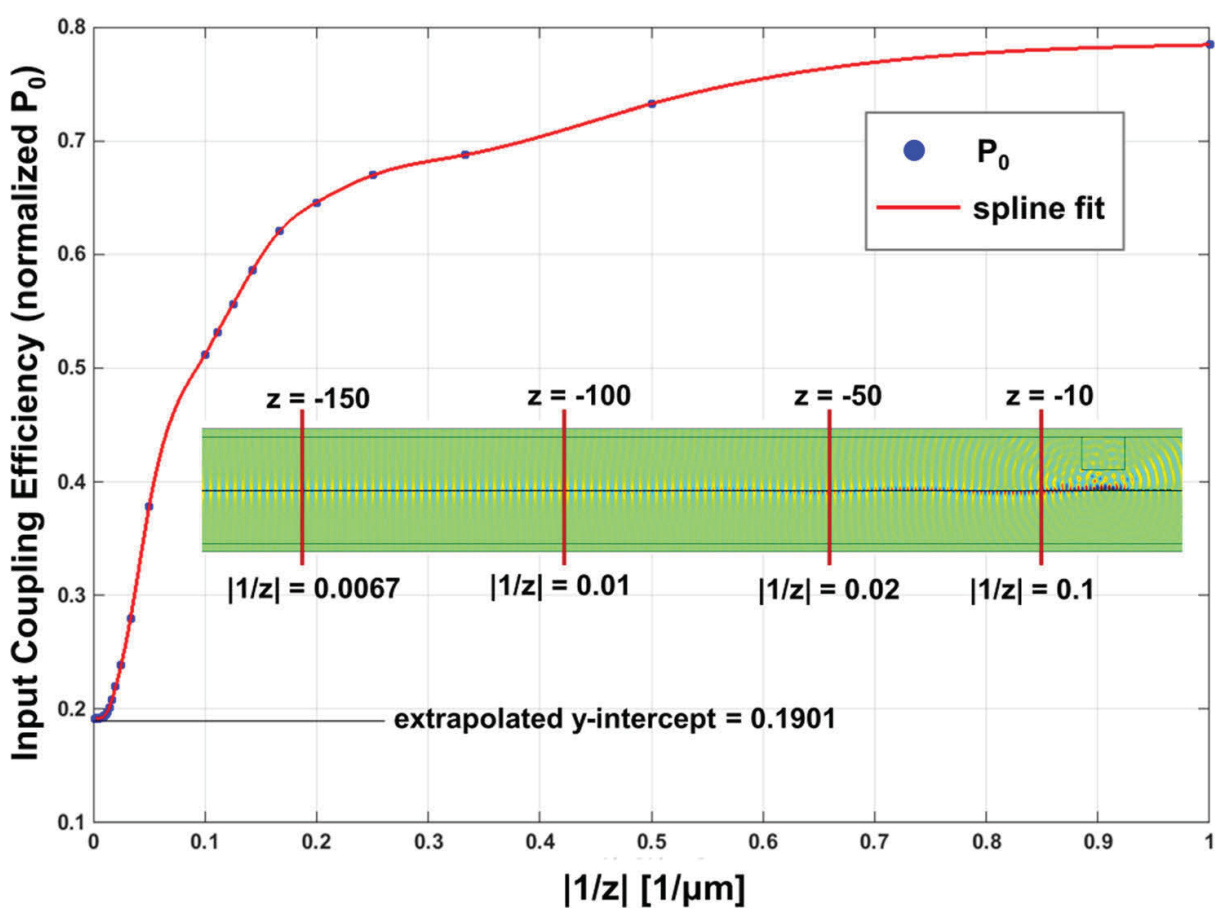

FIG. 11. Computed values for the coupling efficiency $P_{0}$ (normalized). The most accurate value is obtained by extrapolating the curve fit to $|1 / z|=0$. 


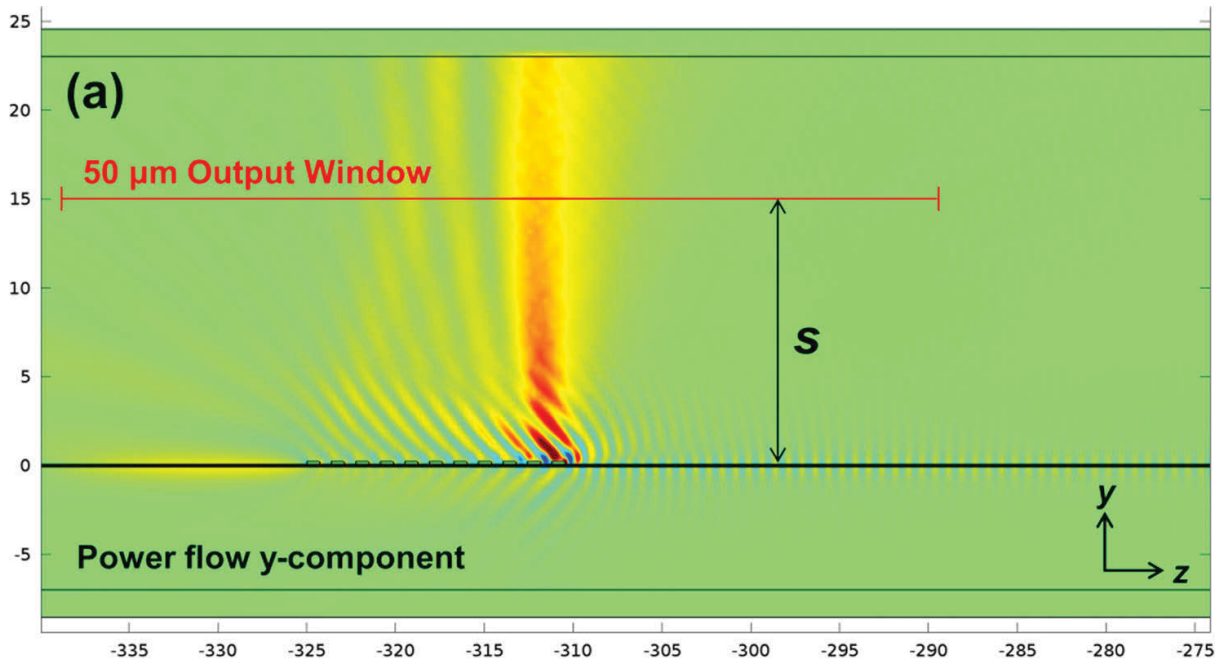

FIG. 12. (a) Output grating scattered power flow (y-component) and (b) zdirection power flow at the output grating.

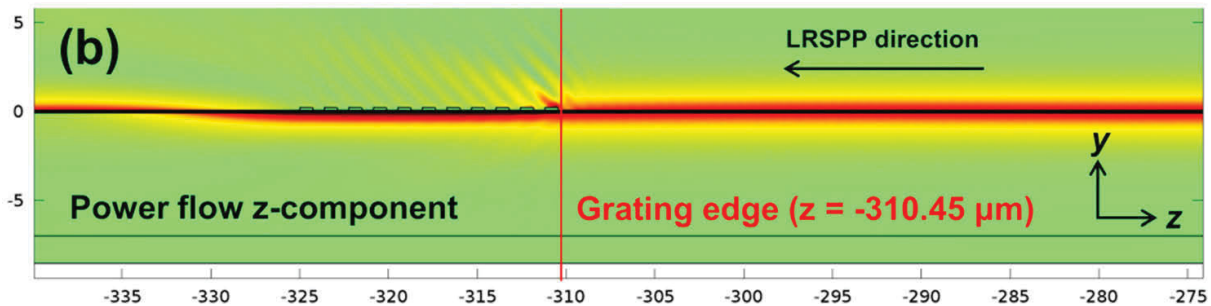

From Fig. 12, it is clear that the output power will also be dependent on the vertical position of the output window $(s)$. Fig. 13 shows the computed coupling efficiency as a function of output height calculated using the extrapolated grating edge power. With the output very close to the grating $(2 \mu \mathrm{m})$, a coupling efficiency of $\sim 0.41$ (coupling loss of $\sim 3.9 \mathrm{~dB}$ ) can be achieved. As the output position is moved further away, it steadies to $\sim 0.28$ or (coupling loss of $5.5 \mathrm{~dB})$ at $20 \mu \mathrm{m}$.

\section{MODE OVERLAP COUPLING EFFICIENCY}

Another important point of analysis is the coupling between the waveguide and sensor LRSPP modes at the palladium patch input and output. The LRSPP will propagate along a gold-only waveguide section prior to interacting with the palladium covered sensor portion. This discontinuity will result in a coupling loss which can be determined by the overlap integral of the two mode field profiles. ${ }^{26}$ An example of the mode discontinuity was previously shown in Fig. 2. The overlap is calculated as

$$
C=\frac{\iint_{A} E_{y 1} \cdot E_{y 2}^{*}}{\sqrt{\left(\iint_{A} E_{y 1} \cdot E_{y 1}^{*}\right)\left(\iint_{A} E_{y 2} \cdot E_{y 2}^{*}\right)}}
$$

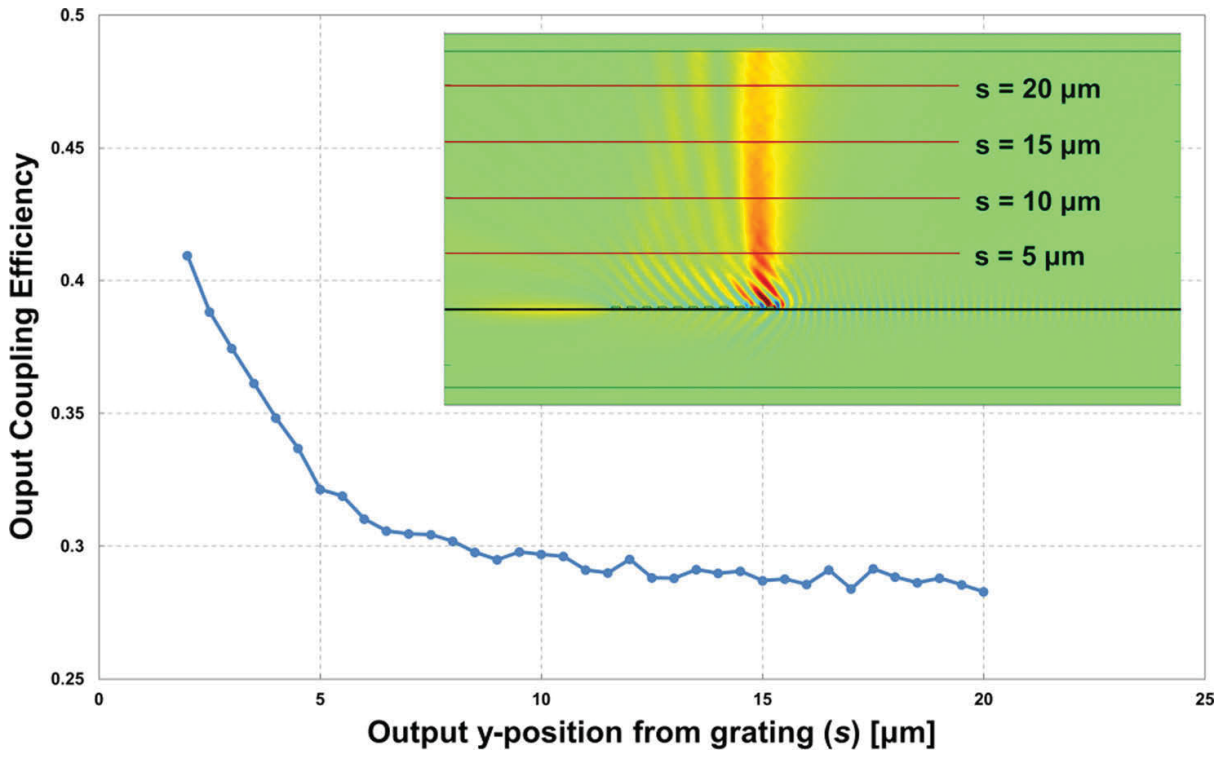

FIG. 13. Plot of the output coupling efficiency computed at various heights (y) from the output grating. 


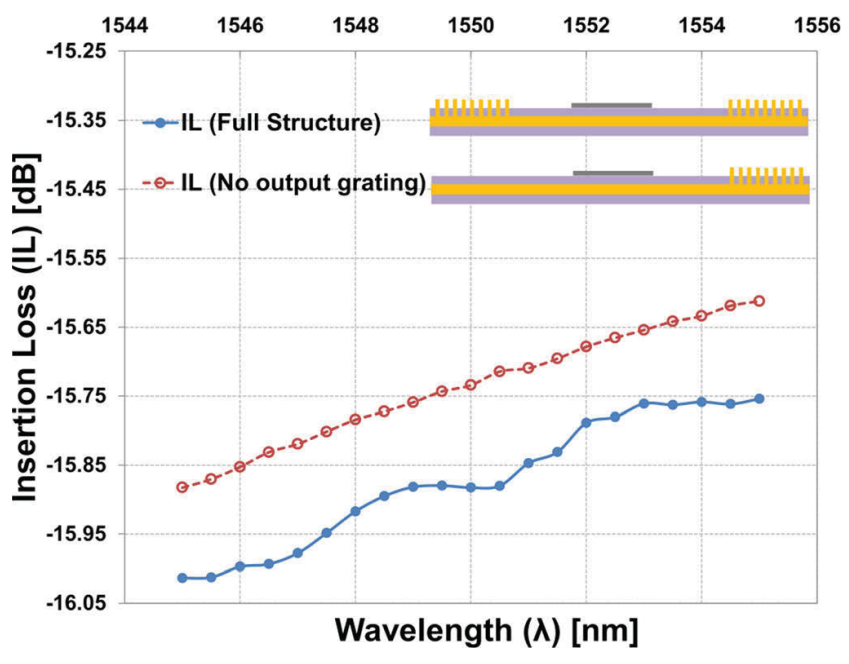

FIG. 14. Simulated wavelength dependency of the full sensor structure (solid) and of the same structure but with one grating coupler removed (no cavity effect).

where $E_{y 1}$ and $E_{y 2}$ are the complex electric field distributions of the two modes. The overlap integral was calculated numerically in MATLAB using exported data from our FEM analysis and determined to be $0.318 \mathrm{~dB}$. Each Pd sensor patch will have one input and one output resulting in double the overlap loss of $0.636 \mathrm{~dB}$.

\section{OPTICAL CAVITY EFFECTS}

The device geometry forms a Fabry-Pérot optical cavity with the input and output gratings behaving as plane-parallel partial reflectors. Thus, a wavelength dependent power oscillation is expected. This effect can be modeled and analyzed by a wavelength sweep of the full device in $2 \mathrm{D}$ using a similar technique as previously shown in Sec. IV, but now inserting a Pd patch. Fig. 14 (solid) shows the computed insertion loss measured just before the output grating as a function of wavelength. The material permittivities are approximated as constant over this small wavelength window. In this figure, it is observed that there is less loss with increasing wavelength (due to the reducing attenuation of the waveguide with increasing wavelength). More importantly, there is a noticeable oscillation caused by the optical cavity.

The discontinuities of the $\mathrm{Pd} / \mathrm{Au}$ and Au-only overlaps may also induce reflections forming a Fabry-Pérot cavity. But this cavity effect is very weak because the reflections are very low, as has been verified by isolating the Pd cavity in the simulation by removing the output grating, thus eliminating the longer cavity. The plot in Fig. 14 (dashed) shows a significant reduction in the oscillation. It can therefore be concluded that the oscillations are caused by the optical cavity formed by the grating couplers.

\section{CONCLUDING REMARKS}

The design space for the LRSPP cladded membrane waveguide was determined along with designs for input and output grating couplers. The waveguide sections have a fairly low MPA of $0.0097 \mathrm{~dB} / 10 \mu \mathrm{m}$ while the sensor sections have $0.55 \mathrm{~dB} / 10 \mu \mathrm{m}$. From the computations involving the gratings, it was shown that the LRSPP mode stabilizes $\sim 100 \mu \mathrm{m}$ from the grating edge. By designing the sensor such that there is a $100 \mu \mathrm{m}$ section of gold-only waveguide next to each grating and a $100 \mu \mathrm{m}$ long sensor patch, the total insertion loss from mode attenuation would be $\sim 5.7 \mathrm{~dB}$. Adding in the mode overlap and best case input and output coupling efficiency the total insertion loss of the device would be $\sim 17.4 \mathrm{~dB}$, which is well within manageable limits using a low power laser diode and a good quality power sensor.

In summary, the full design for a LRSPP cladded membrane waveguide hydrogen sensor operating at $\lambda_{0}=1550 \mathrm{~nm}$ is presented. Broadside grating couplers can provide convenient input and output coupling to the sensor.

${ }^{1}$ J. Tollefson, Nature 464, 1262 (2010).

${ }^{2}$ T. Hübert, L. Boon-Brett, G. Black, and U. Banach, Sens. Actuators, B 157, 329 (2011).

${ }^{3}$ M. A. Butler, Appl. Phys. Lett. 47, 1007 (1984).

${ }^{4}$ J. Villatoro and D. Monzon-Hernandez, Opt. Express 13, 5087 (2005).

${ }^{5}$ B. Sutapun, Sens. Actuators, B 60, 27 (1999).

${ }^{6}$ K. Schroeder, W. Ecke, and R. Willsch, Opt. Laser Eng. 47, 1018 (2009).

${ }^{7}$ P. Tobiška, O. Hugon, A. Trouillet, and H. Gagnaire, Sens. Actuators B 74, 168 (2001).

${ }^{8}$ X. Bévenot, A. Trouillet, C. Veillas, H. Gagnaire, and M. Clément, Meas. Sci. Technol. 13, 118 (2002).

${ }^{9}$ V. N. Konopsky and E. V. Alieva, Opt. Lett. 34, 479 (2009).

${ }^{10}$ N. R. Fong, P. Berini, and R. N. Tait, Sens. Actuators, B 161, 285 (2012).

${ }^{11}$ H. Raether, Surface Plasmons on Smooth and Rough Surfaces and on Gratings (Spring, Berlin, 1988).

${ }^{12}$ A. D. Boardman, Electromagnetic Surface Modes (Wiley, Chichester, 1982).

${ }^{13}$ P. Berini, Adv. Opt. Photon. 1, 484 (2009).

${ }^{14}$ D. Sarid, Phys. Rev. Lett. 47, 1927 (1981).

${ }^{15}$ A. Boltasseva, T. Nikolajsen, K. Leosson, K. Kjaer, M. S. Larsen, and S. I. Bozhevolnyi, J. Lightwave Technol. 23, 413 (2005).

${ }^{16}$ P. Berini, R. Charbonneau, and N. Lahoud, Nano Lett. 7, 1376 (2007).

${ }^{17}$ A. W. Wark, H. J. Lee, and R. M. Corn, Anal. Chem. 77, 3904 (2005).

${ }^{18}$ H. Fan, R. Buckley, and P. Berini, Appl. Opt. 51, 1459 (2012).

${ }^{19}$ O. Krupin, H. Asiri, C. Wang, R. N. Tait, and P. Berini, Opt. Express 21, 698 (2013).

${ }^{20}$ B. Chadwick, J. Tann, M. Brungs, and M. Gal, Sens. Actuators, B 17, 215 (1994).

${ }^{21}$ Z. Zhao, M. A. Carpenter, H. Xia, and D. Welch, Sens. Actuators, B 113, 532 (2006).

${ }^{22}$ M. Wang and Y. Feng, Sens. Actuators, B 123, 101 (2007).

${ }^{23}$ N. R. Fong, P. Berini, and N. Tait, J. Vac. Sci. Technol. B 33, 021201 (2015).

${ }^{24}$ R. Charbonneau, P. Berini, E. Berolo, and E. Lisicka-Shrzek, Opt. Lett. 25, 844 (2000).

${ }^{25}$ T. Nikolajsen, K. Leosson, I. Salakhutdinov, and S. I. Bozhevolnyi, App. Phys. Lett. 82, 668 (2003).

${ }^{26}$ P. Berini, R. Charbonneau, N. Lahoud, and G. Mattiussi, J. Appl. Phys. 98, 043109 (2005).

${ }^{27}$ R. Daviau, E. Lisicka-Shrzek, R. N. Tait, and P. Berini, Appl. Phys. Lett. 94, 091114 (2009).

${ }^{28}$ T. B. Flanagan and W. A. Oates, Annu. Rev. Mater. Sci. 21, 269 (1991).

${ }^{29}$ X. Bévenot, A. Trouillet, C. Veillas, H. Gagnaire, and M. Clément, Sens. Actuators, B 67, 57 (2000).

${ }^{30}$ W. Vargas, I. Rojas, D. Azofeifa, and N. Clark, Thin Solid Films 496, 189 (2006).

${ }^{31}$ E. D. Palik, Handbook of Optical Constants of Solids II (Academic, Boston, 1991).

${ }^{32}$ P. Berini, Phys. Rev. B 61, 10484 (2000).

${ }^{33}$ N. R. Fong, P. Berini, and R. N. Tait, J. Microelectromech. Syst. 19, 700 (2010). 
${ }^{34}$ N. Fong, P. Berini, and R. N. Tait, J. Vac. Sci. Technol. A 27, 614 (2009).

${ }^{35}$ V. N. Konopsky, D. V. Basmanov, E. V. Alieva, D. I. Dolgy, E. D. Olshansky, S. K. Sekatskii, and G. Dietler, New J. Phys. 11, 063049 (2009).
${ }^{36}$ W. J. Buttner, M. B. Post, R. Burgess, and C. Rivkin, Int. J. Hydrogen Energy 36, 2462 (2011).

${ }^{37}$ C. Chen and P. Berini, IEEE Photonics Technol. Lett. 21, 1831 (2009).

${ }^{38}$ C. Chen and P. Berini, Opt. Express 18, 8006 (2010). 


\section{Chapter: Fabrication Methods}

The following article entitled "Fabrication of long-range surface plasmon hydrogen sensors on Cytop membranes integrating grating couplers" was published in Journal of Vacuum Science and Technology B (JVSTB) volume 33 in February 2015 and is presented in full. It was selected as an "Editor's Pick" for the JVSTB website and was a featured article in the American Vacuum Society (AVS) "Beneath the AVS Surface" newsletter.

The article represents work performed solely by the primary author under the supervision and guidance of the co-authors. It details the fabrication methods used to produce the final structures as presented in Chapter 4.

The device was fabricated entirely at Carleton University's microfabrication lab with the exception of the e-beam lithography exposure which was performed at the Berini Lab at the University of Ottawa. 


\title{
Fabrication of long-range surface plasmon hydrogen sensors on Cytop membranes integrating grating couplers
}

\author{
Norman R. Fong a) \\ Department of Electronics, Carleton University, 1125 Colonel By Drive, Ottawa, Ontario K1S 5B6, Canada \\ Pierre Berini ${ }^{\text {b) }}$ \\ School of Electrical Engineering and Computer Science and Department of Physics, University of Ottawa, 161 \\ Louis Pasteur, Ottawa, Ontario K1N 6N5, Canada \\ Niall Tait ${ }^{\mathrm{c})}$ \\ Department of Electronics, Carleton University, 1125 Colonel By Drive, Ottawa, Ontario K1S 5B6, Canada
}

(Received 28 October 2014; accepted 14 January 2015; published 29 January 2015)

\begin{abstract}
The fabrication process for a long-range surface plasmon polariton hydrogen sensor is presented. The device, referred to as the cladded membrane waveguide, features a $5 \mu \mathrm{m}$ wide and $20 \mathrm{~nm}$ thick gold stripe embedded in a $160 \mathrm{~nm}$ free standing Cytop membrane. Broadside excitation and output are achieved with integrated grating couplers. Hydrogen sensitivity is provided by an overlaid $5 \mathrm{~nm}$ thick palladium patch, which acts as a transduction medium. The device is fabricated by integrating several process techniques including blind through-wafer alignment, optical photolithography, overlaid electron beam lithography, metal lift-off, and through-substrate silicon wet etching. Fabricated results are presented along with a detailed discussion. The devices are characterized optically via a cutback measurement with the measured waveguide attenuation being consistent with simulated values. (C) 2015 American Vacuum Society. [http://dx.doi.org/10.1116/1.4906827]
\end{abstract}

\section{INTRODUCTION}

Devices based on surface plasmon resonance (SPR) have become predominant for optical biological, chemical, and gas sensing. ${ }^{1,2}$ This is due to the inherently high surface sensitivity of SPR structures which can produce strong changes in optical intensity in response to analyte interacting with surface chemistry. Hydrogen $\left(\mathrm{H}_{2}\right)$ gas is of particular interest to detect as it is present in many industrial applications such as: chemical plants, nuclear power plants, coal mines, semiconductor manufacturing, and more recently, fuel cells. It is flammable in air at concentrations between $4 \%$ and $75 \%$ and hence the ability to quickly and reliably detect it is a significant safety concern. Optical detection methods provide advantages over electrical or electrochemical hydrogen sensing in that they do not require electrical signals, and hence, there is no risk of ignition within the explosive atmosphere. ${ }^{3}$

A variety of SPR-based hydrogen sensors have been implemented with structures such as the Kretschmann configuration, ${ }^{4}$ planar waveguides, ${ }^{5}$ diffraction gratings, ${ }^{6}$ nanoantennas, ${ }^{7}$ nanoparticles on photonic crystal arrays, ${ }^{8}$ and optical fibers. ${ }^{9-11}$ All of these hydrogen sensors incorporate palladium $(\mathrm{Pd})$ as it selectively and proportionally reacts with hydrogen.

Surface plasmon polaritons (SPPs) are transverse magnetic polarized optical surface waves that propagate along the interface between a semi-infinite dielectric and a metal. The long-range surface plasmon polariton (LRSPP) is a low attenuation mode which can be obtained with a finite thickness and width metal film, symmetrically cladded by a

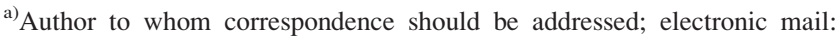
nofong@doe.carleton.ca

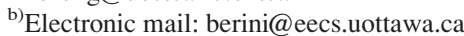

${ }^{c}$ Electronic mail: niall@ doe.carleton.ca
}

uniform dielectric. ${ }^{12}$ For sensing, benefits to LRSPP waveguides over single-interface SPPs are the increased propagation length, leading to longer optical interaction lengths and thus higher sensitivity and the structures can be integrated and mass-manufactured.

Our proposed sensor, diagrammed in Fig. 1, is a membrane-supported LRSPP waveguide. The sensor features a palladium patch on top of a free-standing Cytop (dielectric) membrane with an embedded gold (Au) stripe. Input and output light coupling is achieved through diffraction gratings, which allow broadside excitation and capture directly from optical fibers or focused beams. This article describes and discusses the detailed fabrication process to realize such a device.

\section{DEVICE DESCRIPTION}

\section{A. Structure and materials}

The device builds on a structure referred to as the LRSPP membrane waveguide, which features a metal stripe suspended on a dielectric membrane. ${ }^{13,14}$ By embedding the stripe into the membrane, there is an improved refractive index symmetry, which reduces attenuation. This new structure is referred to as the cladded membrane waveguide. Figure 2 shows a multiperspective schematic of the device. Gold is used for the waveguide as it has relatively long propagation lengths at the desired operating wavelength $(1550 \mathrm{~nm})$. Cytop is a chemically resistant amorphous fluoropolymer, which is used for the membrane as it has a low refractive index $(\sim 1.34)$ and has been demonstrated to work well in other LRSPP devices. ${ }^{15}$ Grating couplers are fabricated directly onto the waveguide and will protrude outwards as diagrammed in Fig. 2(b). The arrows indicate the direction of input, propagated and output light. The devices 


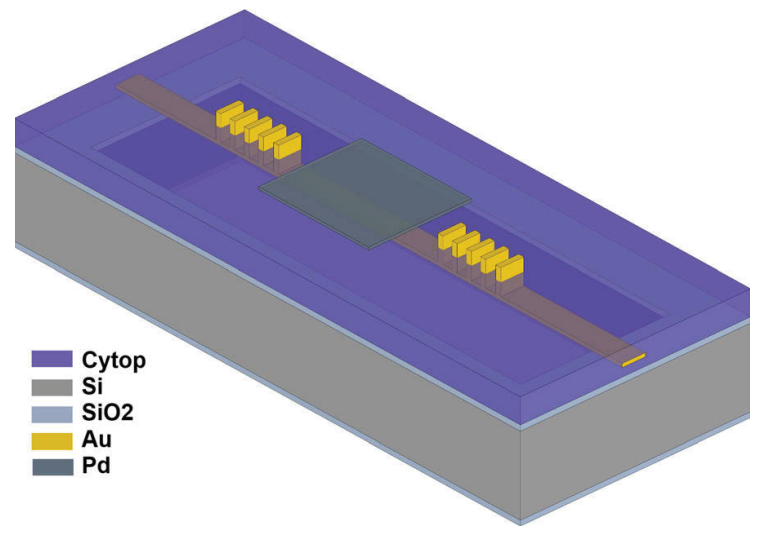

FIG. 1. (Color online) Diagram of the cladded membrane waveguide LRSPP hydrogen sensor. Dimensions are not diagrammed to scale.

are symmetrical and the input and output gratings can be reversed.

\section{B. Sensor operation}

Input light is coupled perpendicularly via the input grating, which is designed to excite the LRSPP mode along the membrane waveguide. The waveguide has a relatively low confinement, which allows the electric field to interact with a section of overlying palladium. Upon hydrogen exposure, this will induce a shift in the mode attenuation, which is measured via scattered light from the output grating. The attenuation sensitivity has been previously explored for a comparable structure, ${ }^{14}$ and this device is predicted to be capable of very low concentration detection.

\section{FABRICATION DETAILS}

\section{A. General process considerations}

The process is founded upon previous knowledge with sensors using Cytop. ${ }^{16-18}$ The membranes for this structure are $\sim 2.5 \times$ thinner than previous Cytop membrane devices, ${ }^{16}$ making them significantly more fragile and thus requiring new process steps for their fabrication. There are two primary restrictions that dictated the evolution of the process flow. (a)
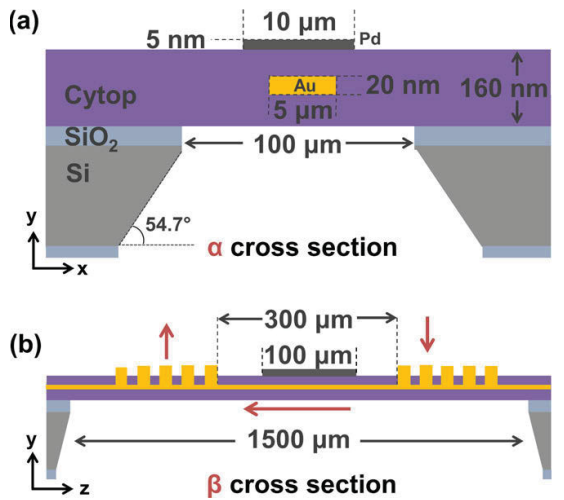

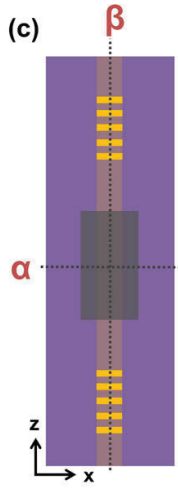

FIG. 2. (Color online) Multiperspective depiction of the cladded membrane waveguide hydrogen sensor. Typical dimensions are identified but exaggerated in the depiction. (a) and (b) Cross sections through cut lines $\alpha$ and $\beta$ diagrammed in (c).
The first is that Cytop has a glass transition temperature $\left(\mathrm{T}_{\mathrm{g}}\right)$ of $107^{\circ} \mathrm{C}$ and will reflow above this temperature. This is a concern since it can cause irreversible deformation of metal features that are deposited on Cytop. ${ }^{17}$ Reflow is more pronounced as the wafer temperature increases above $\mathrm{T}_{\mathrm{g}}$. High temperature process steps are ultimately unavoidable but kept to a minimum. The second consideration is that the membrane will be released with a timed Tetramethylammonium hydroxide (TMAH) silicon wet etch, and therefore, any material used must be chemically compatible with TMAH. The final process steps are diagramed in Fig. 3.

\section{B. Wafer layout and design}

Although all the devices could be fabricated on one large membrane, such a structure would be unnecessarily fragile. Instead, smaller individual membranes $(\sim 100 \mu \mathrm{m} \times 1500 \mu \mathrm{m})$ were designed with multiple devices per membrane. The full wafer mask layout is shown in Fig. 4(a) with a single cell shown in Fig. 4(b). From this layout, three photomasks were made: one for the front-side gold, one for the front-side palladium, and one for the back-side membranes. Since $\langle 100\rangle$ silicon wafers are used, the membrane mask must be larger than the desired membrane dimensions due to the angled sidewalls formed by the TMAH etch.

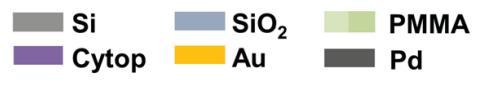

(a)

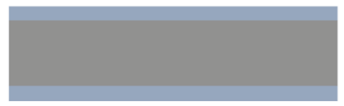

(b)

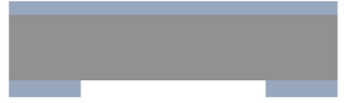

(c)

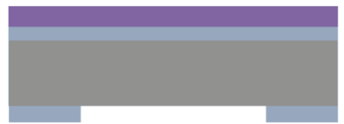

(d)

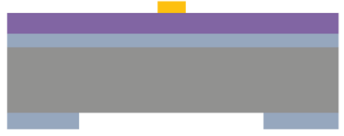

(e)

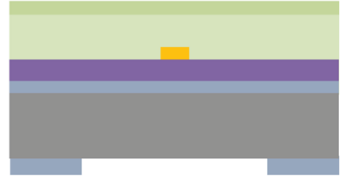

(f)

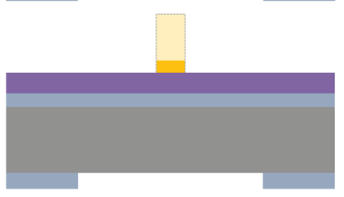

(g)

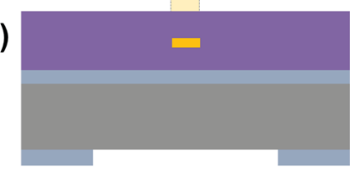

(h)

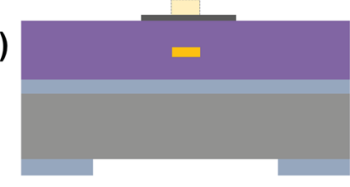

(i)

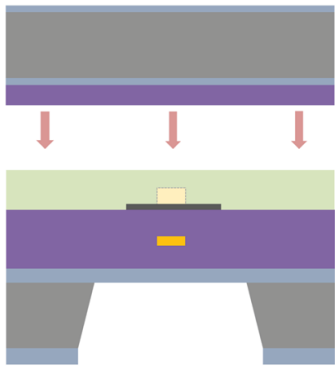

(j)

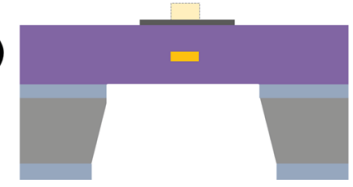

FIG. 3. (Color online) Fabrication flow (a) $\mathrm{SiO}_{2}$ etch stop and mask layers, (b) membrane mask pattern and etch, (c) cytop film coating, (d) Au stripe pattern and deposition, (e) gratings lithography, (f) gratings deposition and lift-off, (g) Cytop top cladding, (h) Pd patch pattern and deposition, (i) wafer passivation and TMAH etch, and (j) removal of sacrificial layers. 


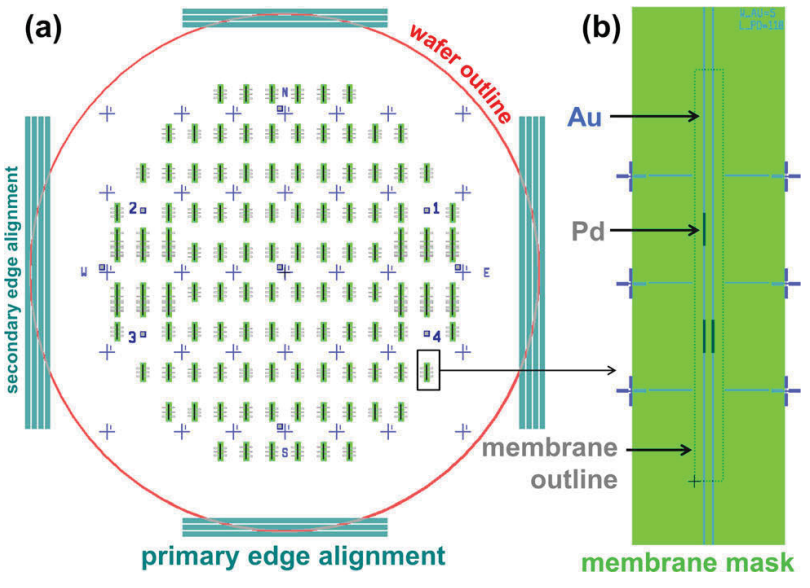

FIG. 4. (Color online) (a) Full wafer mask layout. Edge alignment marks appear on all masks to allow for front-to-back alignment. (b) Expanded single cell layout showing two stripes and multiple sensors per membrane.

In order to fabricate this structure, there needs to be accurate through-wafer alignment between the backside membrane cavity mask and the front waveguide masks. This can be accomplished with a double-sided wafer alignment and exposure system but such a system was not available. Therefore, a blind through-wafer alignment technique was developed. To accomplish this, both backside and front side masks were designed with identical alignment lines along the outer edges. The primary wafer flat can be aligned to the same primary alignment line on each mask while the perpendicular wafer edge is aligned to a secondary edge alignment line on the back mask and then aligned to the corresponding but opposite mark on the front mask. In order for this technique to work the layout needs to be center-symmetric.

\section{Wafer selection and preparation}

The starting wafers are 2 in. $\langle 100\rangle$ single-side polished ntype silicon. The wafer is used solely as physical substrate and does not contribute either optically or electrically and hence its type ( $\mathrm{n}$ or $\mathrm{p}$ ) and resistivity are irrelevant. However $\langle 100\rangle$ p-type wafers with secondary flats can help with alignment. A RCA clean is applied and then $55 \mathrm{~nm}$ of dry thermal silicon dioxide is grown [Fig. 3(a)]. This oxide serves two purposes: on the back side it is patterned into the etch mask for the cavity while on the front side also behaving as an etch stop layer. The etch stop is critical in order to avoid undercutting and potentially delaminating the membrane, as occurred in previous structures. ${ }^{16}$

\section{Backside patterning and etch mask}

Before processing the wafer back side, a protective layer of photoresist is required on the front as this face will eventually be placed down during the preceding processing steps and its preservation is a high priority. First, an adhesion promoter, hexamethyldisilazane (HMDS), is spin coated on the front surface. The spin is applied at $1000 \mathrm{rpm}$ for $10 \mathrm{~s}$ and the wafer is baked at $100^{\circ} \mathrm{C}$ for $1 \mathrm{~min}$ on a hot plate. Next, the protective photoresist (Shipley S1811) is spin-coated $(1000 \mathrm{rpm}$ for $10 \mathrm{~s}$ and then $4000 \mathrm{rpm}$ for $30 \mathrm{~s}$ resulting in in $1.1 \mu \mathrm{m}$ layer) and hard baked at $115^{\circ} \mathrm{C}$ for $5 \mathrm{~min}$. The steps are repeated on the back side of the wafer and it is soft baked at $\left(100^{\circ} \mathrm{C}\right.$ for $\left.1 \mathrm{~min}\right)$. The wafer back side is aligned and exposed using the membrane mask. The photoresist is developed with a TMAH based developer (Shipley MF-321) and hard baked $\left(115^{\circ} \mathrm{C}\right.$ for $\left.3 \mathrm{~min}\right)$. The backside oxide is etched with a reactive ion etch using $\mathrm{CF}_{4} / \mathrm{O}_{2}$ plasma $(200 \mathrm{~W}$ for $140 \mathrm{~s}$ in a March Jupiter II RIE system). The remaining photoresist is removed with acetone and isopropyl alcohol (IPA) with the wafer in the state diagrammed by Fig. 3(b).

\section{E. Front side waveguide lithography}

A thorough surface clean is performed in $\mathrm{O}_{2}$ plasma using a Plasma-Preen microwave plasma cleaning system. Five percentage concentration Cytop M-grade is spun onto the front surface ( $1000 \mathrm{rpm}$ for $10 \mathrm{~s}$ then $5000 \mathrm{rpm}$ for $30 \mathrm{~s}$ ). It is baked on a hot plate at $50{ }^{\circ} \mathrm{C}$ for $30 \mathrm{~min}$ and then ramped to $200^{\circ} \mathrm{C}$ over an hour and held for at least $1 \mathrm{~h}$. This will result in a postcure film thickness of approximately $150 \mathrm{~nm}$ with about $1 \mathrm{~nm}$ of variation across the wafer [Fig. 3(c)].

The film thickness is measured via ellipsometry and then it is etched down to the desired thickness of approximately $70 \mathrm{~nm}$ in $\mathrm{O}_{2}$ plasma using a Technics PEII Planar Plasma Etcher. This thinning step also acts as an ashing step and is necessary for the subsequent photoresist layers to have sufficient adhesion to the Cytop. The plasma etch is not uniform and introduces approximately $5 \mathrm{~nm}$ of long-range thickness variation in the film. The wafers are immediately coated with HMDS using a HMDS vacuum vapor prime oven. Liftoff resist (LOR 1A) is spin coated $(1000 \mathrm{rpm}$ for $10 \mathrm{~s}$ then $4000 \mathrm{rpm}$ for $30 \mathrm{~s}$ ) and baked at $180^{\circ} \mathrm{C}$ for 3 min resulting in $\mathrm{a} \sim 100 \mathrm{~nm}$ film. The photoresist layer ( $\mathrm{S} 1805$ ) is spin coated using the same spin speeds and baked at $115^{\circ} \mathrm{C}$ for $2 \mathrm{~min}$ which form a $\sim 450 \mathrm{~nm}$ layer. The wafers are aligned to the waveguide level mask, exposed, and developed.

Gold $(20 \mathrm{~nm})$ is deposited via thermal evaporation. After deposition, metal lift-off is performed with two static Microposit remover 1165 baths at $80^{\circ} \mathrm{C}$ with a $10 \mathrm{~s}$ ultrasonic agitation in between. The wafers are rinsed with IPA and water. At this point, the structure resembles that sketched in Fig. 3(d).

\section{F. Gratings lithography}

The input and output grating couplers are formed directly onto the gold waveguides with dimensions as diagrammed in Fig. 5. The precision of these dimensions is very strict, so a bilayer electron beam lithography process is used. The bilayer is formed by using two layers of polymethyl methacrylate (PMMA) with different molecular weights, which

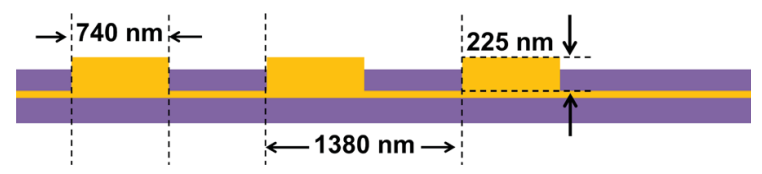

FIG. 5. (Color online) Enlarged side view diagram of the required dimensions for the gratings. 
dissolve at different rates after e-beam exposure and can produce a sufficient undercut for lift-off.

The wafers are coated with HMDS with the vacuum vapor prime and PMMA $495 \mathrm{~K}$ A6 is spin coated onto the surface by ramping up to $1000 \mathrm{rpm}$ over $15 \mathrm{~s}$, spinning at $3000 \mathrm{rpm}$ for $45 \mathrm{~s}$, and then ramping down to a stop over $10 \mathrm{~s}$. This will result in a postcure thickness of approximately $350 \mathrm{~nm}$. PMMA is normally cured at $180^{\circ} \mathrm{C}$ for at least $90 \mathrm{~s}$; however, this will cause severe deformation of the gold waveguides, and hence, it is instead baked at $100{ }^{\circ} \mathrm{C}$ for a minimum of $24 \mathrm{~h}$ to drive out as much solvent as possible. Afterward it is flash baked at $125^{\circ} \mathrm{C}$ for $30 \mathrm{~s}$ and then $180^{\circ} \mathrm{C}$ for $30 \mathrm{~s}$. Using this technique, very mild waveguide deformation is observed. A second layer of PMMA $950 \mathrm{~K} \mathrm{~A} 2$ is spin coated using a $15 \mathrm{~s}$ ramp to $1000 \mathrm{rpm}, 30 \mathrm{~s}$ spin at $1600 \mathrm{rpm}$, and $10 \mathrm{~s}$ ramp down, which will produce a postcure thickness of approximately $100 \mathrm{~nm}$. The second layer is cured in the same manner as the first. The gratings are then exposed using e-beam (accelerating voltage of $10 \mathrm{kV}$ and dose of $277 \mu \mathrm{C} / \mathrm{cm}^{2}$ ). Despite the fact that there are two insulating substrates (oxide and Cytop), no additional charge dissipation techniques were required in order to obtain the desired resolution; however, several iterations were required in order to obtain optimal results. Excessive energy or voltage would cause damage to the underlying Cytop and cause local delamination of the waveguide and grating. After exposure, the wafer is developed in a 1:3 solution of methyl isobutyl ketone to IPA for $120 \mathrm{~s}$ at room temperature. Figure 6 shows a microscope image of a pair of gratings after development. It can be seen that the bottom bump on each set is slightly underdeveloped and there is remaining PMMA residue in the channel. To clear the residue, a $20 \mathrm{~s}$ descum is performed with an $\mathrm{O}_{2}$ RIE at $100 \mathrm{~W}$.

Afterward, $225 \mathrm{~nm}$ of gold is deposited with e-beam evaporation. The gratings lift-off must be done gently to avoid delamination. The wafer is angled vertically in a beaker filled with acetone at room temperature and left for at least $24 \mathrm{~h}$. The beaker needs to be tightly covered to prevent the acetone from evaporating. Lift-off is induced by gently agitating the edges of the wafer with a foam-tipped swab. The

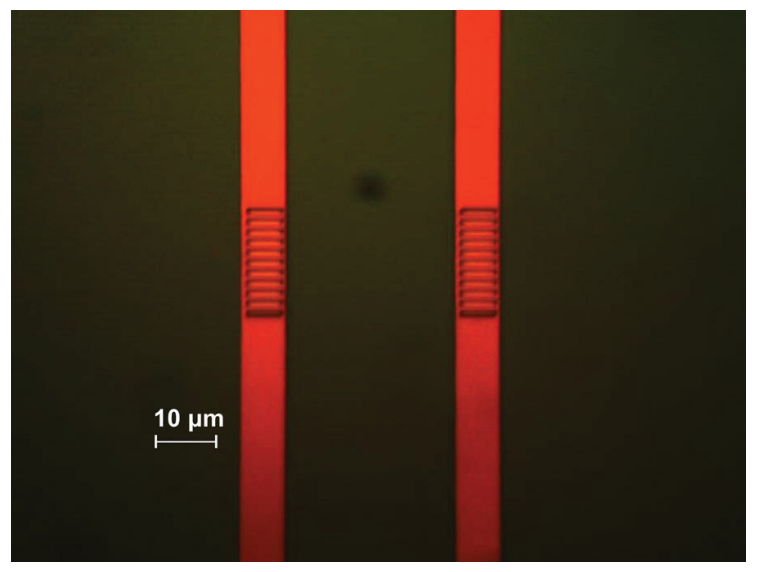

FIG. 6. (Color online) Microscope image of the developed PMMA bi-layer lithography for gratings deposition. wafer is then cleaned with Microposit 1165 followed by IPA. After lift-off, the dimensions can be measured to ensure accuracy and consistency. A SEM image of a grating after lift-off is shown in Fig. 7, which corresponds to Fig. 3(f) in the overall process flow.

\section{G. Top cladding and palladium lithography}

The top Cytop cladding is spin-coated using the same parameters as the lower portion. However, the cladding cannot be cured in the same manner (previously at $180^{\circ} \mathrm{C}$ ) since that process would now result in significant deformation of the waveguides and gratings due to reflow. Instead, a modified hard bake is performed by placing the wafer on a hot plate at $50{ }^{\circ} \mathrm{C}$ for $30 \mathrm{~min}$ and then ramping up to $100^{\circ} \mathrm{C}$ at $5^{\circ} \mathrm{C} / \mathrm{h}$. The wafer is held at this lower baking temperature for a minimum of four days. This increased duration somewhat compensates for the lowered bake temperature but ultimately will not remove as much solvent as the previously applied hard bake. Qualitatively, enough solvent is removed for the film to be solid.

The palladium patches are formed using a similar bilayer lithography process as for the gold waveguides but with some minor modifications. The Cytop stack is etched down to the desired total thickness $(160 \mathrm{~nm})$. HMDS is again applied using the vacuum vapor prime oven. The previous lift-off resist (LOR1A) required a $180^{\circ} \mathrm{C}$ bake for $3 \mathrm{~min}$. Instead, the lift-off resist Polymethylglutarimide (PMGI SFG $2 \mathrm{~S}$ ) is used. PMGI has a slower dissolution rate and therefore can be baked at lower temperatures while still forming an appropriate undercut for the bilayer. The PMGI is spread at $1000 \mathrm{rpm}$ for $10 \mathrm{~s}$ and spun at $4000 \mathrm{rpm}$ spin for $30 \mathrm{~s}$, which results in a $40 \mathrm{~nm}$ thick film. The wafer is baked at $150{ }^{\circ} \mathrm{C}$ for $1 \mathrm{~min}$. This bake causes mild but acceptable deformation in the waveguides. S1805 is used as the photoresist and spin and cured the same way as previously described. The palladium mask is aligned, exposed, and developed. Palladium $(5 \mathrm{~nm})$ is deposited with thermal evaporation and lifted off using the same conditions as the gold lift off described previously. The structure is currently at the state diagrammed in Fig. 3(h). Figure 8 shows microscope images of a pair of waveguides along with two closer views of the gratings and palladium patches. At these thicknesses,

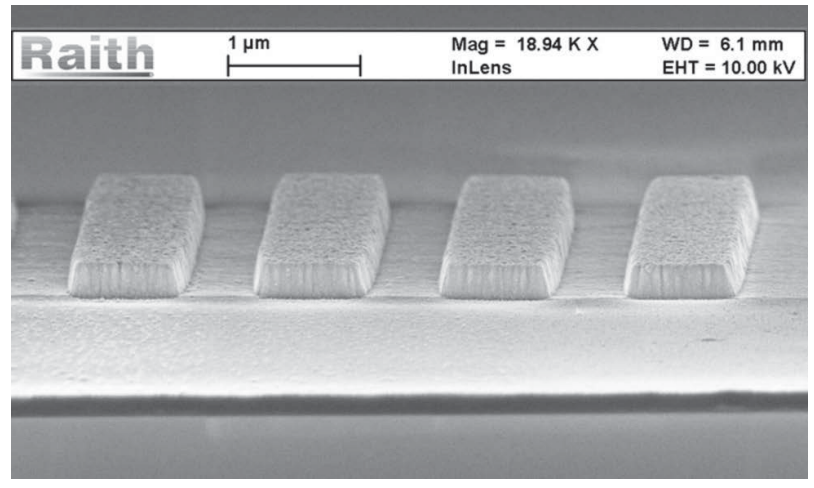

FIG. 7. SEM image of a cleaved section of Au grating on Au waveguide on Cytop with appropriate dimensions. 

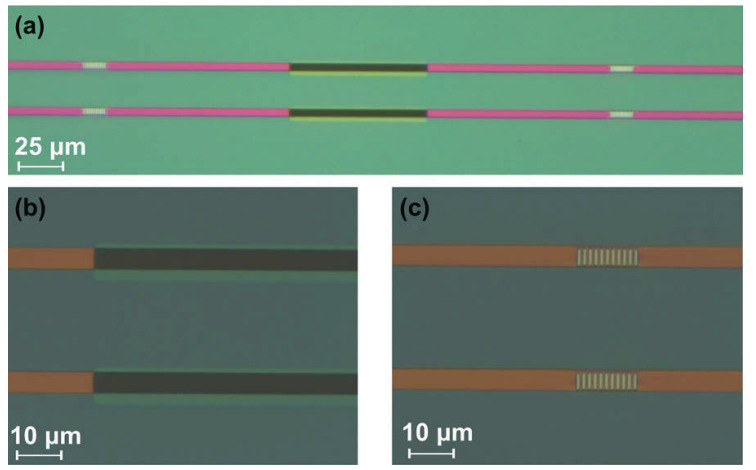

FIG. 8. (Color online) (a) Microscope images showing two full sensors after all lithography has been completed. (b) Expanded view of the palladium overlap. (c) Expanded view of the gratings. Mild but acceptable deformation can be observed.

the gold appears red while the palladium over Cytop over gold produces a dark blue shade.

\section{H. Membrane release}

The membrane is released with a 25\% TMAH silicon wet etch. Although the Cytop film is chemically resistant to TMAH, the wafer cannot be directly exposed to in its current state. TMAH is a strong base and direct exposure to palladium will form palladium hydroxide $\operatorname{Pd}(\mathrm{OH})_{2}$ which not only changes the optical performance of the sensor but also critically deforms its structure. PMMA is resistant to TMAH; therefore, a passivation layer is utilized on the front surface. PMMA $495 \mathrm{~K}$ A6 is spin coated at $1000 \mathrm{rpm}$ for $10 \mathrm{~s}$ then $6000 \mathrm{rpm}$ for $30 \mathrm{~s}$. It is baked at $100^{\circ} \mathrm{C}$ for a minimum of $2 \mathrm{~h}$ and followed with a flash bake at $180^{\circ} \mathrm{C}$ for $30 \mathrm{~s}$. This forms an approximately $300 \mathrm{~nm}$ thick protective film over the surface. It is important to note that this protective film will also induce a stress on the membrane once it has been released and therefore it is generally kept within the thickness range of the membrane.

As previously mentioned, silicon dioxide is used as the cavity etch mask and is known to be resistant to TMAH. It has a slow but nonzero etch rate, which can result in delamination of the Cytop film if the wafer edges are not passivated. This is addressed by using $1 / 4$ in. Teflon (polytetrafluoroethylene) thread tape to wrap the wafer circumference. To ensure that only one face of the wafer is exposed to TMAH, an additional passivation wafer is needed. The passivation wafer is silicon wafer with thick $(\sim 1 \mu \mathrm{m})$ field oxide and a $\sim 2 \mu \mathrm{m}$ thick hard baked Cytop layer as diagrammed in Fig. 3(i). The passivation and membrane wafer are placed face-to-face and edge wrapped again with Teflon thread tape. They are clamped together with Teflon nuts and bolts as shown in Fig. 9. Due to the amount of processing that has occurred on the wafer, the open silicon channels on the back have regrown a native oxide; therefore, a $10 \%$ hydrofluoric acid (HF) swab is applied to the back until the exposed silicon becomes hydrophobic. This needs to be carefully monitored to avoid over exposing the wafer to $\mathrm{HF}$, which can compromise the oxide mask.

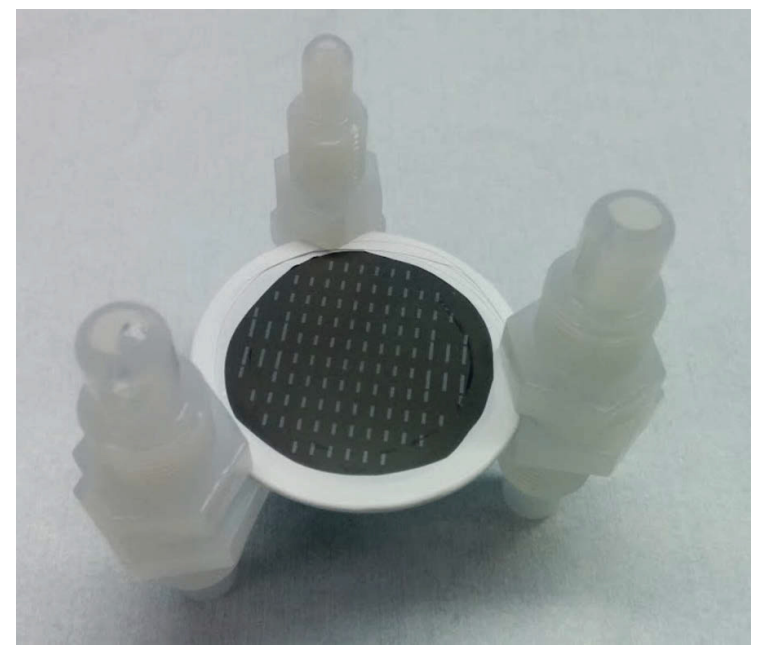

FIG. 9. (Color online) Photograph a single-sided wafer etching setup, before the wet etch. Teflon nuts and bolts are used to clamp the wafer to be etched with another protective wafer. The stack is edge wrapped with Teflon thread tape.

This apparatus is placed into $25 \% \mathrm{TMAH}$ at $65^{\circ} \mathrm{C}$ and can take approximately $24 \mathrm{~h}$ to complete depending on the thickness of the silicon wafer. The etch time can be reduced by increasing the temperature; however, the temperature is intentionally kept low to stay safely within the thermal budget. Upon completion, the wafer is rinsed in two static baths of IPA and allowed to air dry. Figure 10 shows a microscope image at this stage with the PMMA protective layer and oxide etch stop still in place. The oxide causes the visual distortions seen through the now-transparent membrane.

The PMMA protective layer is removed with a static bath of Microposit 1165 at $50^{\circ} \mathrm{C}$ for $5 \mathrm{~min}$ followed by a static IPA bath, IPA spray, and air dry. The final step is to remove the oxide etch stop; this will in turn remove the remaining masking oxide. The wafer is dipped in $10 \% \mathrm{HF}$ for approximately $2 \mathrm{~min}$. It is then immediately immersed in three consecutive IPA baths and allowed to air dry. Figure 11 shows a microscope image of some of the completed structures. It is important to remove the PMMA sacrificial layer first, as the

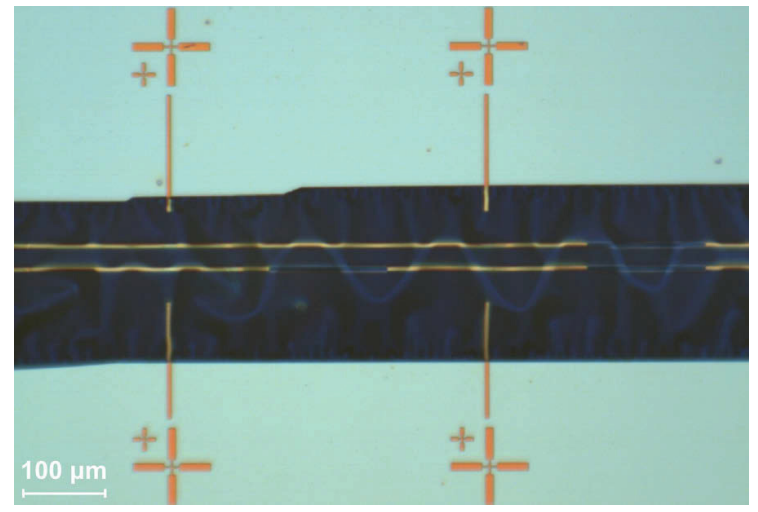

FIG. 10. (Color online) Microscope image of a membrane section after TMAH etching but before the removal of the sacrificial PMMA and oxide etch stop. 


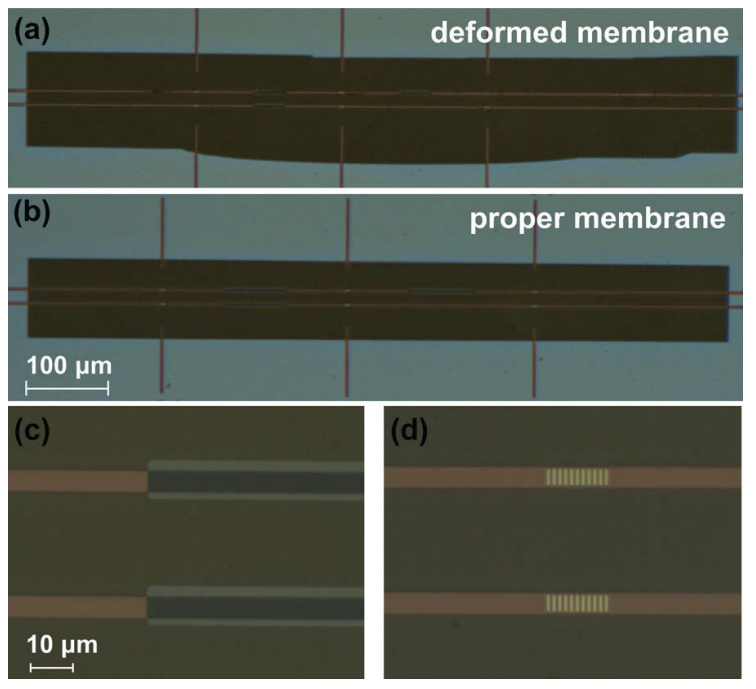

FIG. 11. (Color online) Microscope images of completed membranes. (a) Deformed membrane. (b) Properly released membrane. (c) Expanded view of the palladium patch. (d) Expanded view of gratings.

removal chemistry is more aggressive than that needed for the oxide removal, which will result in significantly poorer yield if the steps are reversed.

\section{RESULTS AND DISCUSSION}

\section{A. Fabrication results}

The membranes should be perfectly rectangular but can occasionally be deformed as shown in Fig. 11(a). Deviations from the rectangular shape can be attributed to misalignments with the backside mask and damage to the backside oxide during processing. Deformed membranes are at higher risk of fracture but should not otherwise negatively impact performance. It can also be observed that the fabricated membrane in Fig. 11(b) is noticeably larger than the expected outline as depicted in Fig. 4(b). This is due to the fact that layout mask was designed for a $300 \mu$ m thick wafer while the depicted wafer was thinner, which will scale the membrane geometry. Also, since this is a timed wet etch, there can be over etching of the sidewalls, which will also increase the size of the window.

The inclusion of an etch stop layer was critical in obtaining consistent results. Without an etch stop, undercutting and delamination of the membrane will quickly occur once the silicon in the membrane cavity is etched. Compounding this problem is the fact that the silicon thickness can vary across the wafer and the etch rate within the TMAH bath can also vary slightly.

Even with an etch stop, there is still a high risk of fracture during the final wafer separation and sacrificial layer removal steps. It was observed that using water to rinse the wafer at any stage after the membranes were released would cause a large number of them to fracture. This is due to the high surface tension of water, which can overly stress a membrane while sheeting off and drying. Instead, IPA is used to rinse as it does not sheet off quickly but rather evaporates slowly leaving the membranes intact. An overall yield

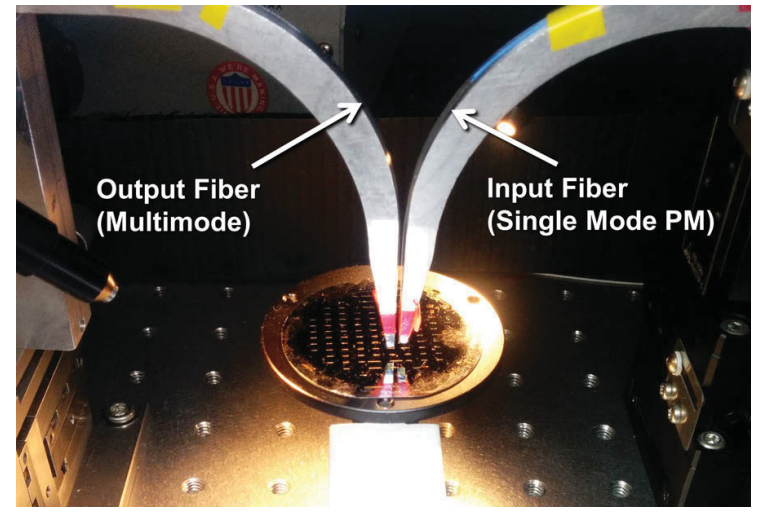

FIG. 12. (Color online) Membrane waveguide optical testing setup. Input and output fibers are mounted onto custom-machined curved arms, which are attached to micropositioners.

of approximately $75 \%-90 \%$ of the membranes per wafer was observed. After drying the membranes were able to be handled easily, without risk of fracture.

\section{B. Optical characterization}

Optical characterization of the structures is performed using a setup as shown in Fig. 12. The wafer is placed on a ring that is attached to a cantilever and mounted to a twoaxis positioner. The input fiber (single mode polarization maintaining) and output fiber (multimode) are mounted onto custom-machined curved supports, which are attached to individual micropositioners for fine alignment.

The wafer layout includes test devices with gold-only waveguides (no palladium) and gratings placed at varying separations, all on one membrane. Figure 13 shows the results of a cutback measurement performed on such a test structure.

From this, the attenuation is determined to be $3.4 \mathrm{~dB} / \mathrm{mm}$, which is about $3.5 \times$ larger than the expected value. This can be attributed to the fact that the fabricated device has a thinner $(\sim 140 \mathrm{~nm}$ instead of $160 \mathrm{~nm})$ and more asymmetric membrane than desired. The discrepancy can be addressed

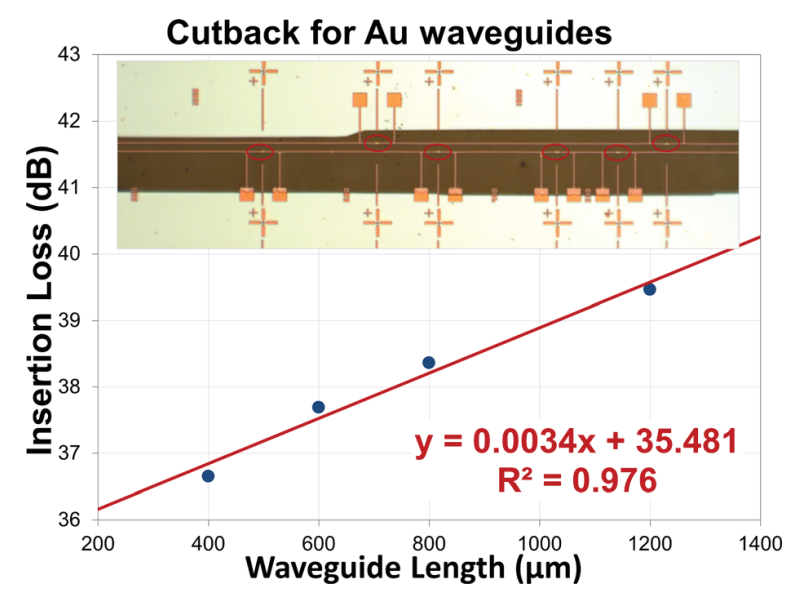

FIG. 13. (Color online) Cutback measurement for characterizing the gold waveguide. (Inset) Microscope image of the cutback structure with the gratings highlighted. 


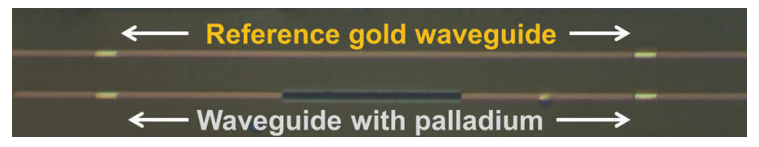

FIG. 14. (Color online) Microscope image of a test structure to determine the insertion loss of the palladium overlay.

by increased process control during the Cytop ashing stages. The simulations were redone for the case of the realized structure dimensions, and the predicted attenuation was $3.1 \mathrm{~dB} / \mathrm{mm}$, which is in excellent agreement with the measured value. The mild deformation to the waveguides during the cladding stage will also contribute some additional loss however this has yet to be quantified. The coupling efficiency $(\sim 35.4 \mathrm{~dB})$ from Fig. 13 was not optimized and thus the coupling loss is large compared to expected values from simulation $(\sim 10 \mathrm{~dB})$. This can generally be improved through more controlled alignment of the fibers, particularly the input fiber height. However, the waveguide attenuation can still be independently extracted from the data.

The effect of the Pd film was characterized by comparing the insertion loss of a gold-only waveguide to that of an equal length waveguide but with a $130 \mu \mathrm{m}$ long Pd overlay as shown in Fig. 14. The difference in insertion loss was measured to be $6.68 \mathrm{~dB}$, which is consistent with the expected excess loss due to the higher attenuation of the $\mathrm{Pd} / \mathrm{Au}$ section and the coupling loss generated at the $\mathrm{Au}$ to $\mathrm{Pd} / \mathrm{Au}$ waveguide junctions.

\section{CONCLUSION}

A full fabrication process was presented for a cladded membrane waveguide hydrogen sensor with broadside grating couplers. The process includes a blind front-to-back alignment along with several optical and e-beam lithography and lift-off steps on Cytop. Membranes are released with a TMAH silicon wet etch. Key factors that improved the overall device yield were the inclusion of a silicon dioxide etch stop layer as well as the use of IPA for rinse steps instead of water. The process has yielded a consistently large number of intact devices which can be used as hydrogen sensors. A cutback measurement of the waveguides shows that the gold sections and palladium/gold sections perform as expected based on comparisons with computations.

\section{ACKNOWLEDGMENTS}

The authors would like to acknowledge Rob Vandusen, Angela McCormick, and Rick Adams from the Carleton University Microfabrication Facility along with Ewa Lisicka-Skrzek and Anthony Olivieri from The University of Ottawa for their assistance and advice on this project.

${ }^{1}$ J. Homola, Anal. Bioanal. Chem. 377, 528 (2003).

${ }^{2}$ J. Homola, Chem. Rev. 108, 462 (2008).

${ }^{3}$ T. Hübert, L. Boon-Brett, G. Black, and U. Banach, Sens. Actuators, B 157, 329 (2011).

${ }^{4}$ B. Chadwick, J. Tann, M. Brungs, and M. Gal, Sens. Actuators, B 17, 215 (1994).

${ }^{5}$ P. Tobiška, O. Hugon, A. Trouillet, and H. Gagnaire, Sens. Actuators, B 74, 168 (2001).

${ }^{6}$ K. Lin, Y. Lu, J. Chen, R. Zheng, P. Wang, and H. Ming, Opt. Express 16, 18599 (2008).

${ }^{7}$ N. Liu, M. L. Tang, M. Hentschel, H. Giessen, and A. P. Alivisatos, Nat. Mater. 10, 631 (2011).

${ }^{8}$ V. N. Konopsky, D. V. Basmanov, E. V. Alieva, S. K. Sekatskii, and G. Dietler, Appl. Phys. Lett. 100, 083108 (2012).

${ }^{9}$ X. Bévenot, A. Trouillet, C. Veillas, H. Gagnaire, and M. Clément, Meas. Sci. Technol. 13, 118 (2002).

${ }^{10}$ A. Hosoki, M. Nishiyama, H. Igawa, A. Seki, Y. Choi, and K. Watanabe, Sens. Actuators, B 185, 53 (2013).

${ }^{11}$ C. Perrotton, R. J. Westerwaal, N. Javahiraly, M. Slaman, H. Schreuders, B. Dam, and P. Meyrueis, Opt. Express 21, 382 (2013).

${ }^{12}$ P. Berini, Adv. Opt. Photonics 1, 484 (2009).

${ }^{13}$ P. Berini, R. Charbonneau, and N. Lahoud, IEEE J. Sel. Top. Quantum Electron. 14, 1479 (2008).

${ }^{14}$ N. R. Fong, P. Berini, and R. N. Tait, Sens. Actuators, B 161, 285 (2012).

${ }^{15}$ O. Krupin, H. Asiri, C. Wang, R. N. Tait, and P. Berini, Opt. Express 21, 698 (2013).

${ }^{16}$ N. Fong, P. Berini, and R. N. Tait, J. Vac. Sci. Technol., A 27, 614 (2009).

${ }^{17}$ C. Chiu, E. Lisicka-Skrzek, R. N. Tait, and P. Berini, J. Vac. Sci. Technol., B 28, 729 (2010).

${ }^{18}$ R. Daviau, A. Khan, E. Lisicka-Skrzek, R. N. Tait, and P. Berini, Microelectron. Eng. 87, 1914 (2010). 


\section{Chapter: Optical Characterization of LRSPP Waveguides}

The following article entitled "Characterization of Grating-Coupled Long Range Surface Plasmon Polariton Membrane Waveguides" was published as an open access research article in Optics Express volume 23 in June 2015 and is presented in full.

The article represents work performed solely by the primary author under the supervision and guidance of the co-authors. It details the methodology and results of the passive optical testing and characterization of the fabricated waveguides. The characterization was performed entirely at the Berini Lab at the University of Ottawa. 


\title{
Characterization of grating-coupled long range surface plasmon polariton membrane waveguides
}

\author{
Norman R. Fong, ${ }^{1, *}$ Pierre Berini, ${ }^{2,3,4}$ and R. Niall Tait ${ }^{1}$ \\ ${ }^{1}$ Department of Electronics, Carleton University, Ottawa, Ontario, K1S 5B6, Canada \\ ${ }^{2}$ School of Electrical Engineering and Computer Science and Department of Physics, University of Ottawa, Ottawa, \\ Ontario, K1N 6N5, Canada \\ ${ }^{3}$ Department of Physics, University of Ottawa, Ottawa, Ontario, K1N 6N5, Canada \\ ${ }^{4}$ Centre for Research in Photonics, University of Ottawa, Ottawa, Ontario, KIN 6N5, Canada \\ *nofong@doe.carleton.ca
}

\begin{abstract}
The first demonstration of grating-coupled long range surface plasmon polaritons in cladded free-standing membrane waveguides is presented. Two different waveguide structures are explored: the first is a gold $\mathrm{C} \mathrm{Au})$ stripe embedded in a thin Cytop free-standing membrane, the other being the same structure but with a thin palladium (Pd) over-layer. The waveguides are excited with integrated grating couplers designed for a working wavelength of $1550 \mathrm{~nm}$. The waveguides are characterized by applying a cutback technique with the Au waveguide loss measured as 3.4 $\mathrm{dB} / \mathrm{mm}$ and the $\mathrm{Pd} / \mathrm{Au}$ waveguide loss as $57 \mathrm{~dB} / \mathrm{mm}$. The wavelength dependency of the weakly reflecting optical cavity is also observed with a free spectral range of $\sim 3.6 \mathrm{~nm}$ and a finesse of 2.1.
\end{abstract}

(C)2015 Optical Society of America

OCIS codes: (050.1950) Diffraction gratings; (240.6680) Surface plasmons; (130.3120) Integrated optics devices; (230.7390) Waveguides, planar; (250.5403) Plasmonics.

\section{References and links}

1. W. L. Barnes, "Surface plasmon-polariton length scales: a route to sub-wavelength optics," J. Opt. A, Pure Appl. Opt. 8(4), S87-S93 (2006).

2. D. Sarid, "Long-range surface-plasma waves on very thin metal films," Phys. Rev. Lett. 47(26), 1927-1930 (1981).

3. P. Berini, "Plasmon-polariton waves guided by thin lossy metal films of finite width: bound modes of symmetric structures," Phys. Rev. B 61(15), 10484-10503 (2000).

4. P. Berini, "Long-range surface plasmon polaritons," Adv. Opt. Photon. 1, 484-588 (2009).

5. P. Berini, R. Charbonneau, and N. Lahoud, "Long-range surface plasmons on ultrathin membranes," Nano Lett. 7(5), 1376-1380 (2007).

6. P. Berini, R. Charbonneau, and N. Lahoud, "Long-range surface plasmons along membrane-supported metal stripes," IEEE J. Sel. Top. Quantum Electron. 14(6), 1479-1495 (2008).

7. N. R. Fong, P. Berini, and R. N. Tait, "Modeling and design of hydrogen gas sensors based on a membranesupported surface plasmon waveguide," Sensor Actuat. Biol. Chem. 161, 285-296 (2012).

8. N. R. Fong, P. Berini, and R. N. Tait, "Modeling of long range surface plasmon polariton cladded membrane waveguides with integrated grating couplers as hydrogen sensors," J. Appl. Phys. 117(16), 163108 (2015).

9. H. Fan, R. Buckley, and P. Berini, "Passive long-range surface plasmon-polariton devices in Cytop," Appl. Opt. 51(10), 1459-1467 (2012).

10. B. Banan, M. S. Hai, E. Lisicka-Skrzek, P. Berini, and O. Liboiron-Ladouceur, "Multi-channel transmission through a gold strip plasmonic waveguide embedded in Cytop," IEEE Photon. J. 5(3), 2201811 (2013).

11. H. Fan and P. Berini, "Thermo-optic characterization of long-range surface-plasmon devices in Cytop," Appl. Opt. 52(2), 162-170 (2013).

12. O. Krupin, H. Asiri, C. Wang, R. N. Tait, and P. Berini, "Biosensing using straight long-range surface plasmon waveguides," Opt. Express 21(1), 698-709 (2013).

13. N. R. Fong, P. Berini, and R. N. Tait, "Fabrication of long-range surface plasmon hydrogen sensors on Cytop membranes integrating grating couplers," J. Vac. Sci. Technol. B 33(2), 021201 (2015).

14. R. Charbonneau, P. Berini, E. Berolo, and E. Lisicka-Shrzek, "Experimental observation of plasmon polariton waves supported by a thin metal film of finite width," Opt. Lett. 25(11), 844-846 (2000). 
15. T. Nikolajsen, K. Leosson, I. Salakhutdinov, and S. I. Bozhevolnyi, "Polymer-based surface-plasmon-polariton stripe waveguides at telecommunication wavelengths," Appl. Phys. Lett. 82(5), 668-670 (2003).

16. P. Berini, R. Charbonneau, N. Lahoud, and G. Mattiussi, "Characterization of long-range surface plasmonpolariton waveguides," J. Appl. Phys. 98(4), 043109 (2005).

\section{Introduction}

Surface Plasmon Polaritons (SPPs) are electromagnetic modes that can propagate along the interface between a dielectric medium and a metal. They exist as a coupled excitation comprised of a TM-polarized electromagnetic wave and a charge density wave in the conductor. A plane interface between a metal and dielectric can allow for guiding of these modes although high attenuation is observed. SPP propagation is strongly sensitive to surface conditions which can be exploited to make highly sensitive sensors. However, large attenuation ultimately limits practical application [1]. The attenuation can be reduced by multiple orders of magnitude by implementing a structure where a thin metal film is symmetrically bounded on both sides by semi-infinite uniform dielectrics [2]. This structure supports what is referred to as a Long Range Surface Plasmon Polariton (LRSPP) which can be used to implement devices having a longer optical interaction with the sensing medium. The structure can be further modified by introducing lateral confinement by limiting the width of the metal film (forming a stripe) [3] thus allowing for the fabrication of integrated optical plasmonic components that can be structured for various applications [4].

The main constraint for LRSPP-based devices is the requirement to ensure refractive index symmetry. For any functional implementation, the propagating electric field needs to be able to interact with the analyte of interest so full encasement in the dielectric is impractical. It has been shown that LRSPPs can propagate on a metal stripe supported by an ultrathin freestanding dielectric membrane [5]. This structure, referred to as the membrane waveguide, allows for sensing environment to bound the structure thus ensuring approximate refractive index symmetry. By functionalizing the metal surface the waveguide can be adapted for biological or gas sensing applications [6,7]. The membrane waveguide can be further improved by embedding the metal directly into the thin membrane and functionalizing a portion of the overlying cladding. This structure is referred to as the cladded free-standing membrane waveguide [8] whose operation and experimental characterization forms the objective of this paper.

\section{Device structure}

\subsection{Cladded membrane waveguide}

Figure 1 shows a depiction of the cladded membrane waveguide along with cross sectional views and the desired dimensions labelled. The waveguide is formed by embedding a gold $\mathrm{Au}$ ) stripe in a thin, free-standing Cytop membrane. Cytop is chemically resistant amorphous fluoropolymer with low refractive index $(\sim 1.335)$ and has been previously demonstrated with other LRSPP devices [8-12]. Input and output light coupling is achieved through the use of grating couplers which allow for broadside (perpendicular) excitation from focused beams or optical fibers. The device in air can operate as a gas sensor by functionalizing a region on top of the waveguide.

For example, a thin patch of palladium $(\mathrm{Pd})$ can be used, which allows this structure to function as a hydrogen sensor, as Pd absorbs hydrogen selectively. However, the device can be modified to sense other gaseous analytes by replacing the $\mathrm{Pd}$ patch with another transduction medium. The device was designed for operation at an operating wavelength of $1550 \mathrm{~nm}$. It was fabricated by first spin-coating and curing the lower portion of the Cytop membrane which is then plasma etched down to the desired thickness. The Au waveguide was formed with a bi-layer lift-off lithography process. 


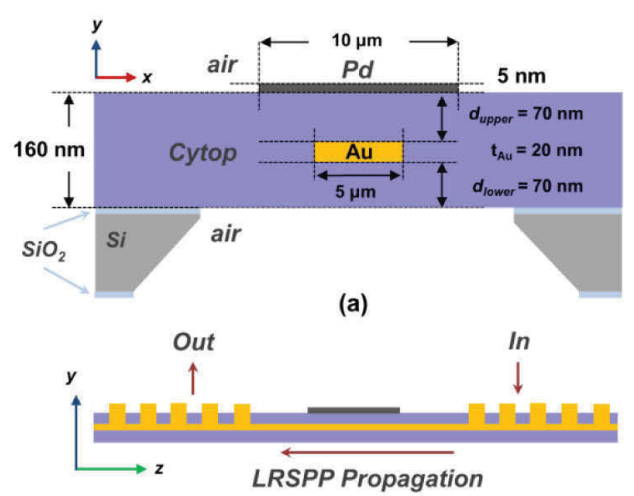

(b)

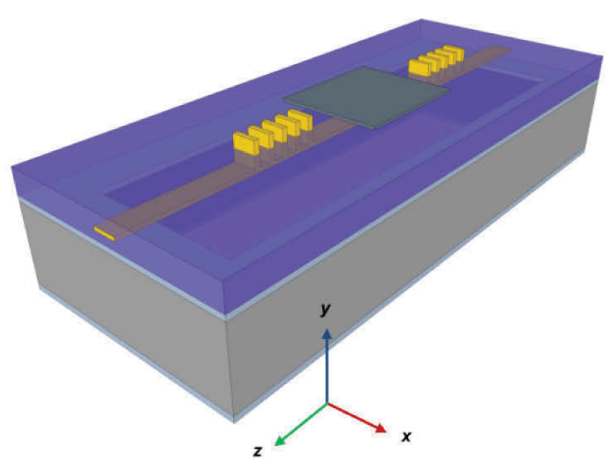

(c)

Fig. 1. (a) A cross sectional depiction through the $x-y$ plane with typical dimensions identified (not drawn to scale). (b) $y-z$ plane cross section showing the gratings geometry (c) 3-D depiction of a single waveguide with $\mathrm{Pd}$ patch

Afterwards the upper Cytop layer was spin coated over the Au and cured. It is then etched to the desired thickness and the Pd deposited, again via lift-off lithography. All devices were fabricated on a 2 inch $<100>$ silicon wafer and a through-wafer wet etch was used to release the membranes using silicon dioxide $\left(\mathrm{SiO}_{2}\right)$ as a mask. The substrate is entirely for structural purposes and does not contribute to the optical operation. Additional fabrication details can be found in [13].

\subsection{Grating couplers}

Input and output light coupling is usually achieved through end-fire excitation from optical fibers. This can allow nearly $100 \%$ coupling efficiency [14-16], however this requires precisely cleaved or diced end facets which would be extremely difficult to achieve without compromising the structural integrity of the membrane. This structure therefore uses grating couplers integrated directly onto the waveguides as depicted. Broadside coupling allows for simpler alignment with flat optical fibers with the tradeoff being lower coupling efficiency. The gratings are formed via electron beam lithography and deposited before the Cytop top cladding is applied [13].

\subsection{Cell layout}

Figure 2(a) shows a photograph of a fabricated wafer. Figure 2(b) shows a microscope image of a single membrane cell $\left(\sim 100 \times 1500 \mu \mathrm{m}^{2}\right)$ with two parallel Au stripes separated by 30 $\mu \mathrm{m}$. All gratings are identical (such that the input and output positions can be interchanged) and are fabricated in the positions indicated, defining 4 individual devices. One section is a Au-only reference waveguide while the remaining three devices have identical Pd patches along their paths. The devices with the Pd are considered to have three distinct regions: a $\mathrm{Pd} / \mathrm{Au}$ waveguide and two surrounding Au-only waveguide sections, which will be individually characterized in Section 4. 

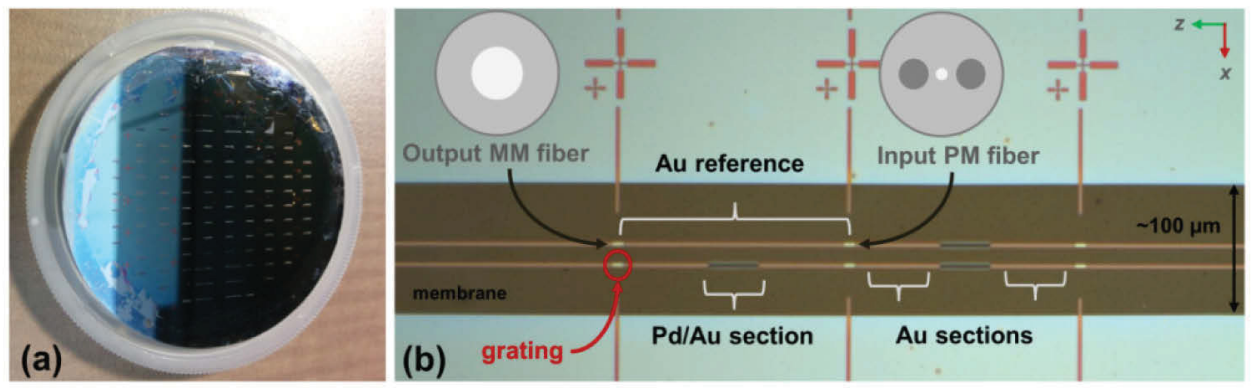

Fig. 2. (a) Photograph of a finished 2 inch wafer. (b) Cell view under microscope. The lighter shaded surface is Cytop over silicon while the dark area is the free-standing membrane. Each cell contains four devices, three of which have Pd patches.

\section{Experimental setup}

The setup for optical characterization of a single device is simple although unconventional. A curved aluminum arm was machined and mounted onto a precision six-axis micro-positioner as shown in Fig. 3(a). A laser diode source at $\sim 1550 \mathrm{~nm}$ is connected to a polarization maintaining (PM) single mode fiber that is mounted with the polarization axis orientated along the waveguide direction as indicated in Fig. 2(b). The output fiber is a multimode fiber, which is mounted on another curved arm, and is connected to an optical power meter (EXFO PM-1600). To ensure minimal back reflection, the wafer is not placed on an opaque surface; rather it is mounted on a ring which is attached to a cantilever mounted to a large range of motion positioner as shown in Fig. 3(b).
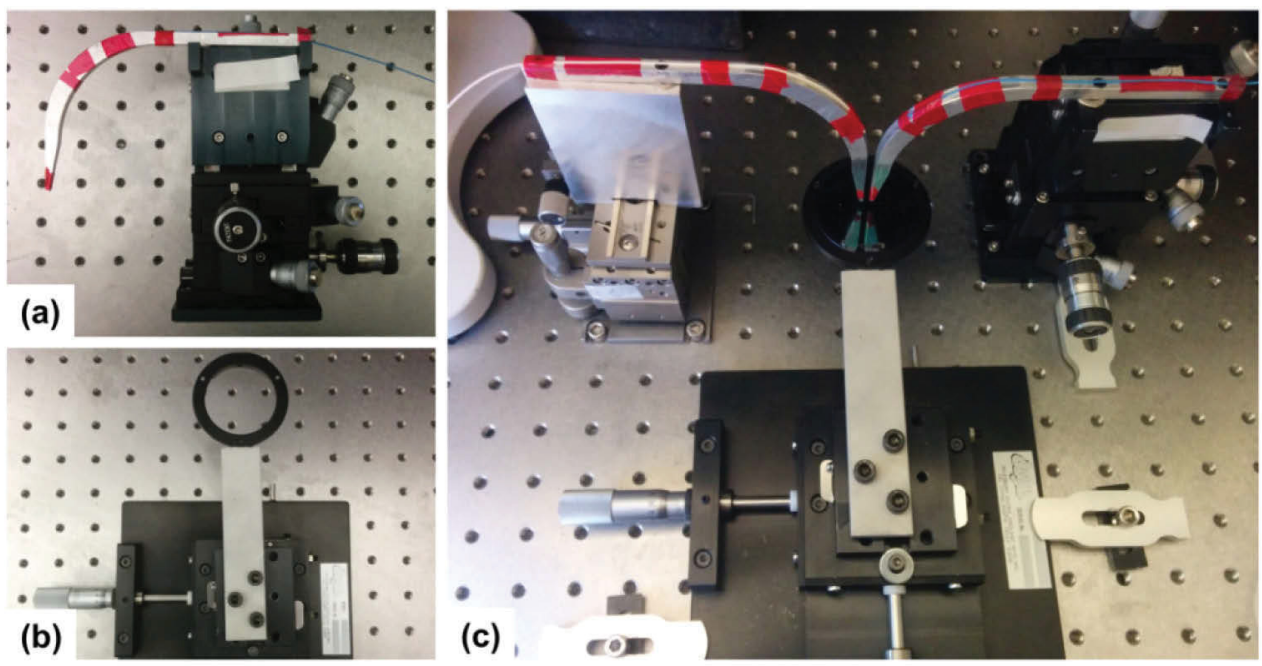

(b)

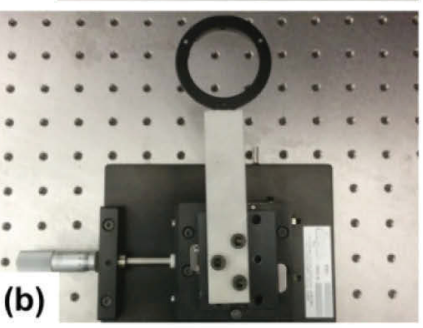

(c)

Fig. 3. (a) Curved beam support attached to a micro-positioner with mounted input PM optical fiber. (b) Large range of motion cantilever with ring chuck. (c) Testing assembly with wafer in place.

The fibers are roughly aligned over the gratings under microscope before the laser source is enabled and the fiber positions are finely aligned to obtain maximum output signal power. A dark board was placed underneath the sample to prevent back-reflections from the optical table. 


\section{Optical characterization}

\subsection{Au waveguides}

There are two distinct waveguide structures to be characterized. The first being the Au stripe in Cytop without a Pd patch (which will be referred to as the Au waveguide) and the second being the region where the Pd overlay is present (referred to as the $\mathrm{Pd} / \mathrm{Au}$ waveguide). Each of these regions supports different LRSPP modes, having significantly different attenuation that needs to be independently quantified. The Au waveguides are characterized via a cutback technique using a cell as shown in Fig. 4(a) where identical gratings are placed at varying separations. Figures 4(b)-4(d) shows the results of measurements taken from three different cutback cells all from the same wafer. A linear fit is applied to the data and the $R^{2}$ coefficient of determination is computed showing strong agreement.
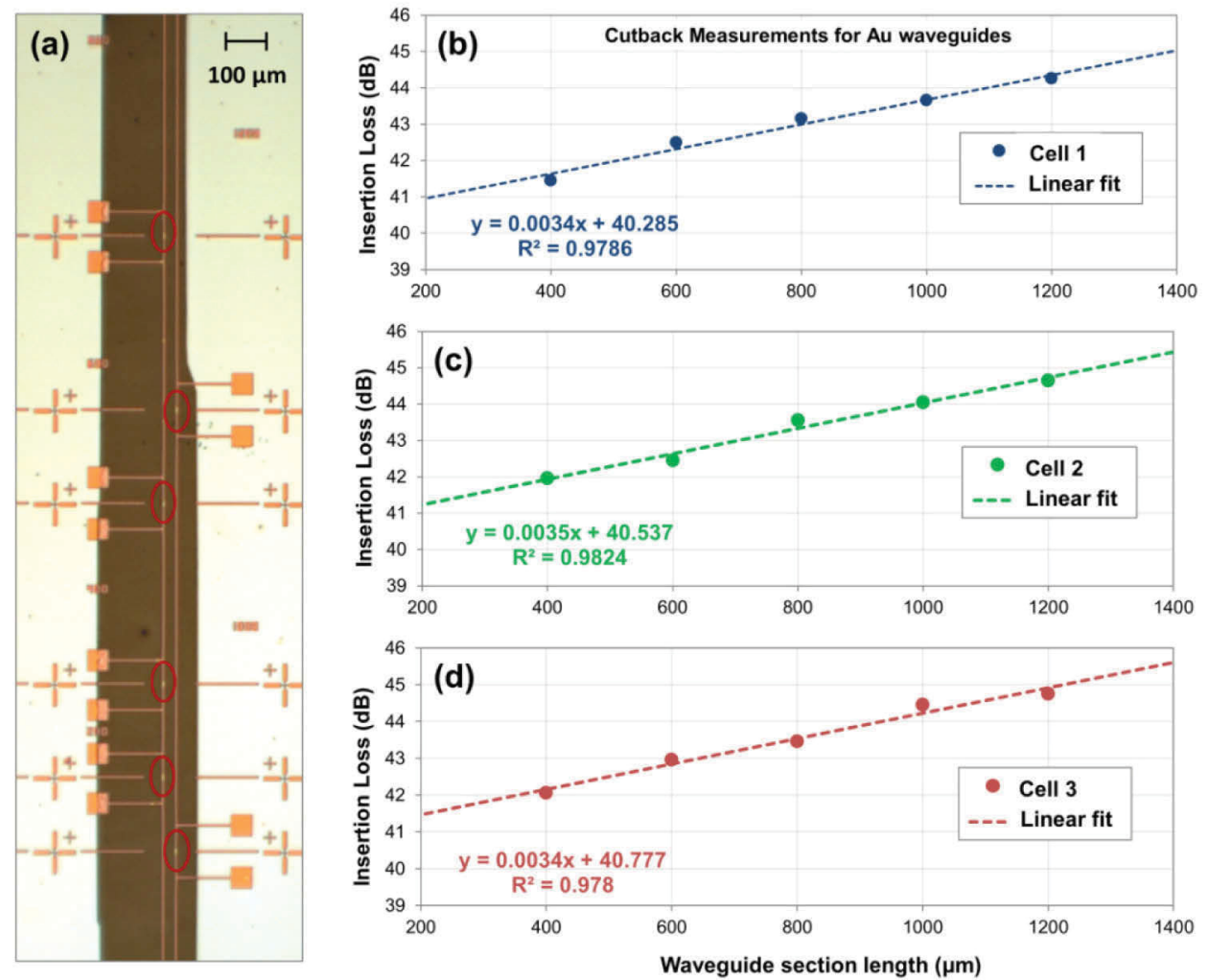

Fig. 4. (a) Microscope image of a cutback cell with varying lengths of Au between gratings. (b)-(d) Cutback measurements from three different samples from different portions of the wafer for determining the mode attenuation of the Au-only membrane waveguide.

The input and output coupling is strongly dependent on distance of the input/output fibers from the wafer. For these experiments the fibers were held relatively far away $(\sim 30 \mu \mathrm{m})$ to ensure consistent, although non optimized coupling. Thus, all of the cutback measurements show large coupling losses ( $y$-intercept), however the slope which represents the mode power attenuation (MPA) can still be independently extracted. From the experimental data the MPA of the Au-only cladded membrane waveguide is $3.4 \mathrm{~dB} / \mathrm{mm}$. This measured attenuation is $\sim 3.5 \times$ larger than that expected from modelling $(0.97 \mathrm{~dB} / \mathrm{mm})[8]$. 


\subsection{Fabricated waveguide dimensions}

The waveguide properties are highly sensitive to the physical dimensions of the metal and membrane and thus the observed discrepancies are likely attributed to geometrical differences between the fabricated devices and the modeled ones. Ideally, the thickness of Cytop above the stripe should be equal to that of the Cytop film beneath $\left(d_{\text {upper }}=d_{\text {lower }}=70 \mathrm{~nm}\right)$. Fabrication errors ultimately resulted in a slightly asymmetrical waveguide. The actual thickness of each layer was measured away from the membrane via ellipsometry during the fabrication. For the wafer tested in the previous section they were measured as $d_{u p p e r}=50 \mathrm{~nm}$ $d_{\text {lower }}=81 \mathrm{~nm}$. However, there may still be small variations in the Cytop thickness across the wafer due to uneven coating and etching.

This thickness asymmetry alone does not fully account for the discrepancy. The devices were examined under atomic force microscope (AFM) to better determine the realized dimensions. Figure 5(a) shows the AFM results for a Au waveguide before the top cladding was applied and Fig. 5(b) shows a scan over the Pd patch of a final device.

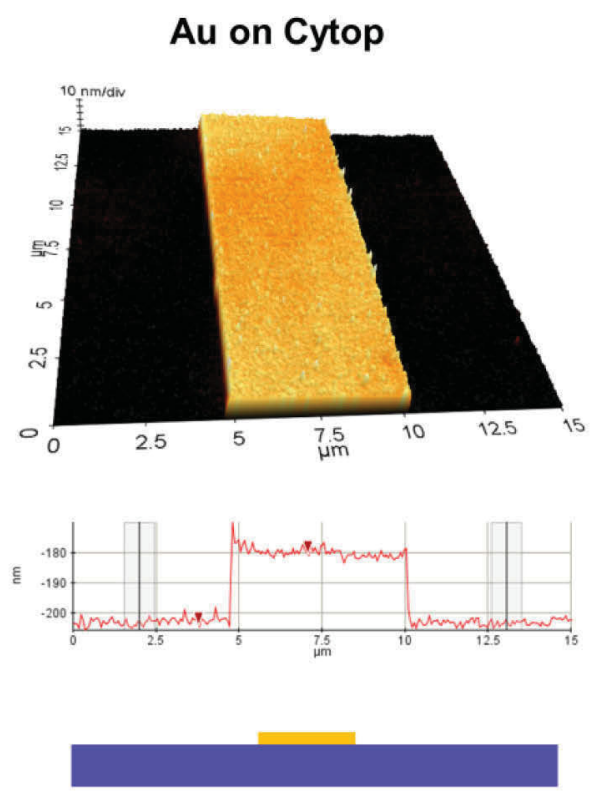

(a)
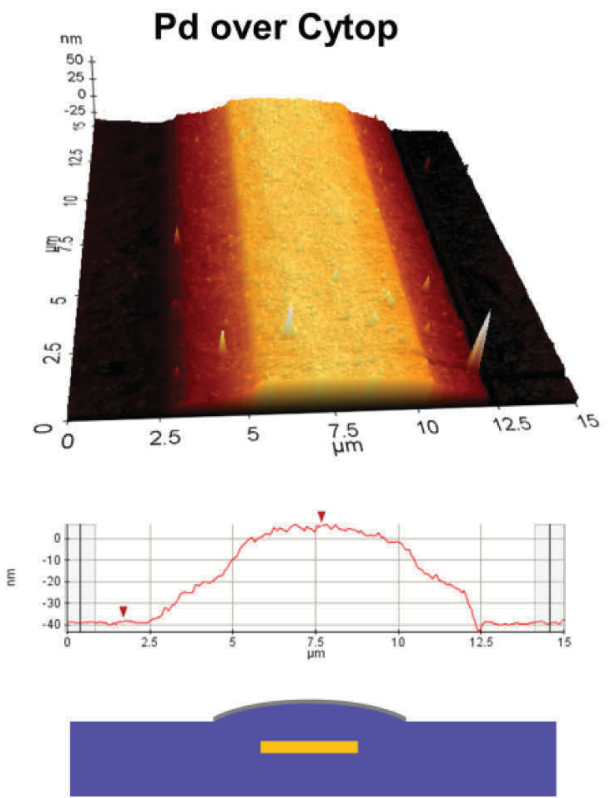

(b)

Fig. 5. AFM scans of (a) an uncladded Au waveguide and (b) a Pd patch section showing significant topography underneath.

From Fig. 5(a) the Au stripe has a thickness of $23.5 \mathrm{~nm}$ ( $20 \mathrm{~nm}$ expected), a width of 5.3 $\mu \mathrm{m}\left(5 \mu \mathrm{m}\right.$ expected) and a root mean squared (RMS) surface roughness of $R_{q}=1.51 \mathrm{~nm}$. Figure 5(b) shows that the surface profile of the Pd patch is rounded over a bump of height $\sim 44 \mathrm{~nm}$. The Pd film was only intended to be about $5 \mathrm{~nm}$ thick which implies that the Cytop upper cladding layer must be causing the topography. Topography is not unexpected since the top layer is applied via spin coating over the Au waveguide without planarization.

Due to the large deviation of the realized dimensions from the intended ones, the original design predictions [8] need to be updated. Figure 6 shows a comparison of the finite element method (FEM) computed mode profile ( $y$-component of the electric field) between an ideally symmetric $\mathrm{Au}$ waveguide structure with exact dimensions and the realized Au waveguide with the previously measured stripe and membrane dimensions. The bump over the waveguide was approximated as a trapezoid. 
The computed MPA of the realized structure $(2.4 \mathrm{~dB} / \mathrm{mm})$ is noticeably higher than that of the ideal device $(0.97 \mathrm{~dB} / \mathrm{mm})$ and reasonably consistent with the measured value of 3.4 $\mathrm{dB} / \mathrm{mm}$. The additional discrepancy can likely be attributed to slight variations in the dimensions from device-to-device. In addition, the surface roughness of the $\mathrm{Au}$, although small, may induce some additional scattering loss.

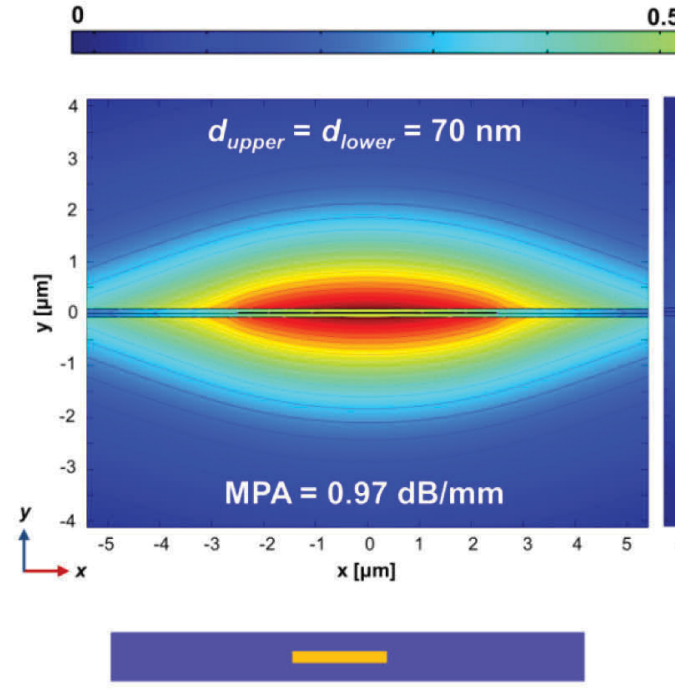

(a)

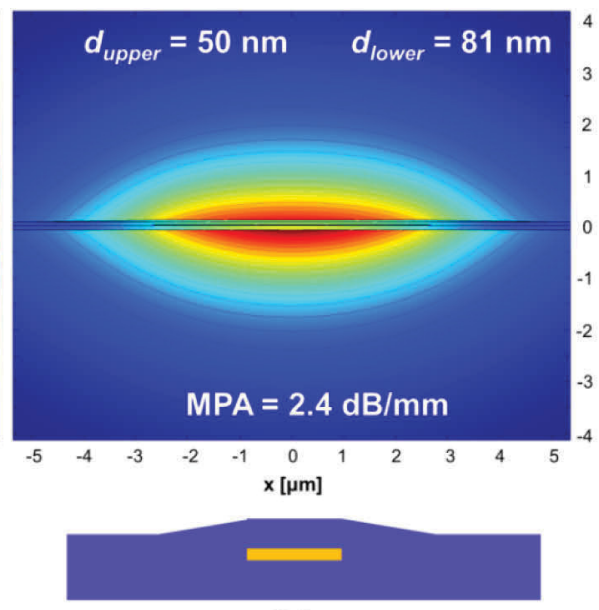

(b)

Fig. 6. Au membrane waveguide mode profiles (Ey) computed via FEM for (a) the intended structure with exact dimensions, and (b) a fabricated structure with asymmetric cladding and topography.

\section{$4.3 \mathrm{Pd} / \mathrm{Au}$ waveguides}

The Pd/Au waveguides can also be characterized with a cutback technique but a simple cutback structure is not available. Instead, each cell was fabricated with different Pd patch lengths, and individually measured with the results combined into a cutback.

This cutback is less straightforward than in the previous case because the loss of a $\mathrm{Pd} / \mathrm{Au}$ section needs to be de-embedded from the Au waveguide sections at the input and output. The cutback is performed by measuring the difference in insertion loss between a total waveguide section with $\mathrm{Pd}\left(I L_{\text {total }}\right)$ and the reference waveguide $\left(I L_{R E F}\right)$ as depicted in inset to Fig. 7 . The insertion loss of the reference waveguide is expressed in $\mathrm{dB}$ as:

$$
I L_{\text {REF }}=\left(C P L_{I N}+C P L_{\text {OUT }}\right)+M P A_{A u}\left(L_{1}\right)
$$

where $C P L_{I N}$ and $C P L_{O U T}$ are the coupling losses of the input and output gratings, while $M P A_{A u}$ is the mode power attenuation of the Au waveguide and $L_{l}$ is its length.

The insertion loss of the device with $\mathrm{Pd}\left(I L_{\text {total }}\right)$, is expressed as:

$$
I L_{\text {total }}=\left(C P L_{I N}+C P L_{O U T}\right)+M P A_{A u}\left(L_{1}-L_{P d}\right)+M P A_{P d}\left(L_{P d}\right)+2 C P L_{P d}
$$

where $M P A_{P d}$ is the MPA of the $\mathrm{Pd} / \mathrm{Au}$ section and $C P L_{P d}$ is the coupling loss that occurs at the discontinuities between the $\mathrm{Au}$ waveguide and the $\mathrm{Pd} / \mathrm{Au}$ section. The difference in insertion loss is obtained by subtracting Eq. (1) from Eq. (2) resulting in:

$$
I L_{\text {total }}-I L_{R E F}=M P A_{P d}\left(L_{P d}\right)+2 C P L_{P d}-M P A_{A u}\left(L_{P d}\right)
$$


This difference represents the insertion loss effect from the Pd overlay only. To compute the MPA, we need to calculate the insertion loss of the $\mathrm{Pd} / \mathrm{Au}$ waveguide section $\left(I L_{P d}\right)$. This can be achieved by adding in the term $M P A_{A u}\left(L_{P d}\right)$ which in the loss of the Au waveguide under the length of Pd, where $M P A_{A u}$ was determined in Subsection 4.1. Figure 7 gives the cutback plot using the computed values of $I L_{P d}$ for various Pd lengths. The slope is the isolated MPA of the $\mathrm{Pd} / \mathrm{Au}$ waveguide, which works out to $\mathrm{MPA}_{\mathrm{Pd}}=57 \mathrm{~dB} / \mathrm{mm}$ while the intercept represents the total coupling losses, $2 C P L_{P d}=0.4875 \mathrm{~dB}$.

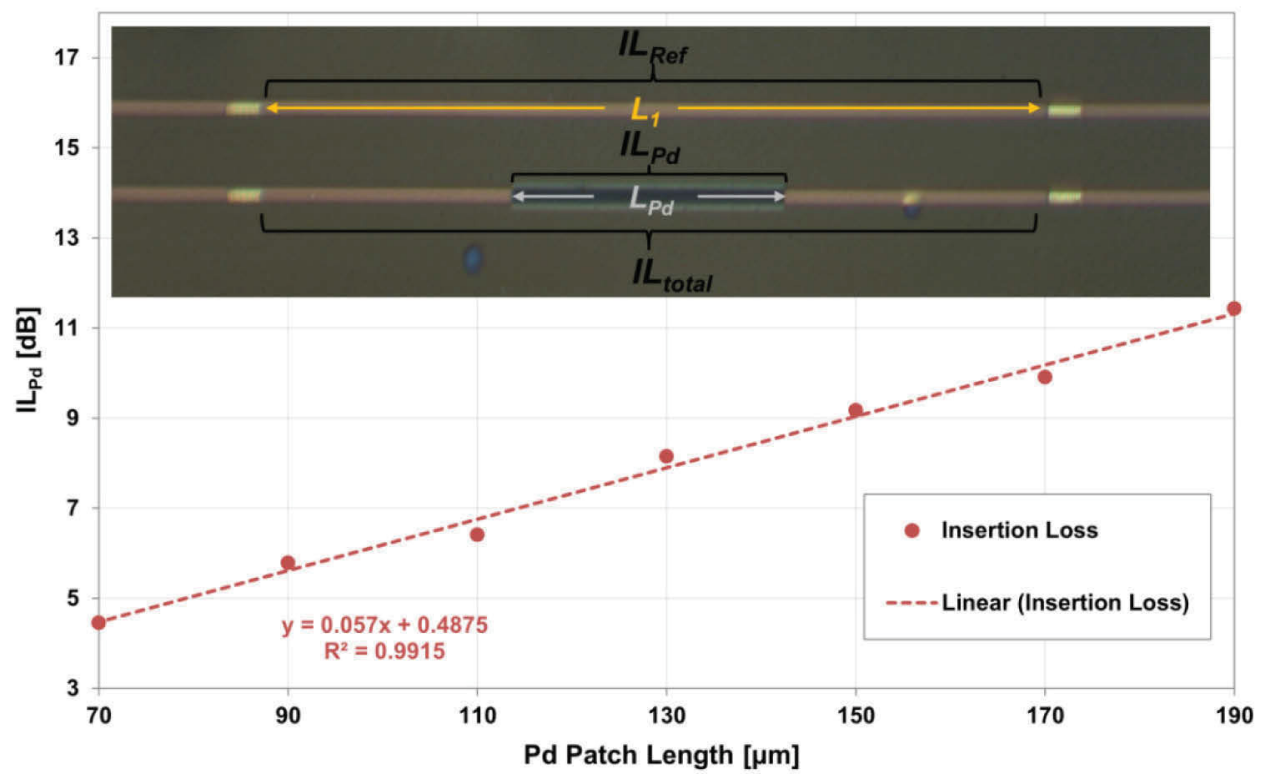

Fig. 7. Plot of the cutback measurements for the $\mathrm{Pd} / \mathrm{Au}$ waveguide. Measurements are taken as the difference in insertion loss between the $\mathrm{Pd} / \mathrm{Au}$ structure and Au-only waveguide, as depicted in the inset.

As discussed in Section 4.2, the actual topography of the realized waveguide is rounded. The deposited Pd film was intended to be $\sim 5 \mathrm{~nm}$ but with the large underlying topography $(\sim 44 \mathrm{~nm})$ it is difficult to accurately isolate the Pd film thickness within the AFM scans. An estimate can be made by simulating various Pd thicknesses on the known membrane topography to attempt to match the measured value. A Pd film of $\sim 3.5 \mathrm{~nm}$ would correspond with the measured result but this is not very conclusive as the permittivity in the infra-red of sub-20 nm metal films can deviate substantially from bulk values used in the model [16].

\subsection{Coupling loss}

From Eq. (3), the $y$-intercept in Fig. 7 is the loss contribution due to mode mismatch ( $\left.2 C P L_{P d}\right)$ occurring at the input and output of the $\mathrm{Pd} / \mathrm{Au}$ section. The theoretical coupling loss can be calculated using an overlap integral as described in $[8,16]$. Using the fabricated dimensions, the total coupling loss has been calculated to be $2 C P L_{P d}=0.35 \mathrm{~dB}$ (or $0.175 \mathrm{~dB}$ per interface), which is slightly less than the observed $\sim 0.4875 \mathrm{~dB}$. The slight discrepancy can be explained by the previously discussed issues with modeling thin Pd layers as well as the aforementioned device-to-device variations. Although it is quantifiable, the coupling loss contributes an insignificant amount to the total loss 


\section{Gratings characterization}

\subsection{Coupling efficiency}

The input and output grating coupling efficiency cannot be easily or independently characterized since they cannot be isolated for testing. However, if carefully aligned insertion loss measurements are performed on a Au-only waveguide, the combined input and output coupling efficiency can be estimated by subtracting out the known waveguide loss.

The gratings have been previously modeled [8] yielding an expected input coupling loss of $5.7 \mathrm{~dB}$ and an output coupling loss of $3.8 \mathrm{~dB}$ for a total coupling loss of $9.5 \mathrm{~dB}$. Several insertion loss measurements of Au-only waveguides of known length were taken, and the total coupling loss was extracted, resulting in an average measured total coupling loss of 18.4 $\mathrm{dB}$, which is significantly higher than predicted.

However, the design modeling [8] was performed in 2-D (infinite $x$-direction) and overestimates the coupling efficiency. The typical 1/e mode field diameter for a single-mode $(1550 \mathrm{~nm}) \mathrm{PM}$ fiber is about $10 \mu \mathrm{m}$, which is significantly wider than the width of the waveguide $(5 \mu \mathrm{m})$. Therefore without beam focusing, a fair amount of beam power will not be incident on the grating resulting in additional uncoupled power.

Figure 8 shows an AFM image over a section of a grating along with a profile plot along the indicated line. It is clear that the infilling of the space between each Au grating bump is not flat as sketched in Fig. 1(b), but rather forms a rounded valley. For this wafer, the Au bumps should protrude by $\sim 175 \mathrm{~nm}$ from the Cytop, whereas the measured peak-to-valley height is $161 \mathrm{~nm}$. These factors explain the deviations between the measured and modelled results.

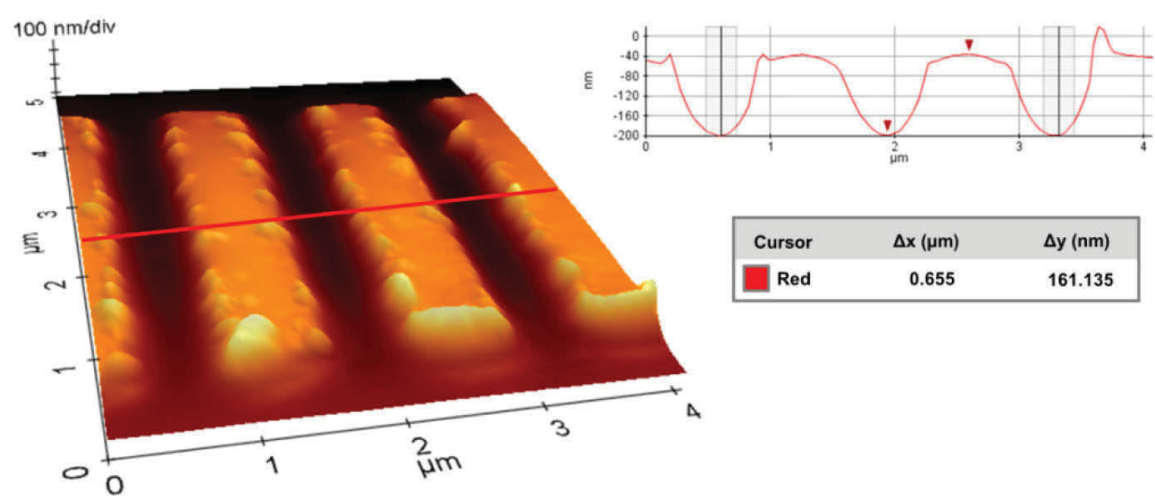

Fig. 8. AFM scan over a sample region of a grating showing a curved Cytop infill between the grating bumps along with residue along the Au edges.

The AFM scan also clearly shows significant residue along the edges of the protruding $\mathrm{Au}$ bumps. This residue did not appear prior to the application of the upper cladding and it is presumed to be residual Cytop. This residue likely contributes to additional coupling loss due to scattering. Despite the increased coupling loss over the predicted, the power is still well within detectable levels, even when using low power input laser sources.

\subsection{Wavelength response}

The grating wavelength response can be characterized by utilizing a tunable wavelength laser source and a broadband optical power detector. Figure 9 shows the insertion loss of a $\mathrm{Au}$ waveguide as the wavelength is varied between 1540 to $1560 \mathrm{~nm}$. An obvious wavelength dependent oscillation is observed. This can be explained by the Fabry-Pérot resonance formed in the optical cavity due to back reflections from the gratings as sketched in inset to Fig. 9. The free spectral range (FSR) of an optical cavity is calculated as: 


$$
F S R=\lambda_{0}^{2} / 2 n_{e f f} d
$$

where $n_{\text {eff }}$ is the effective index of the waveguide, computed as 1.0096 at $1550 \mathrm{~nm}$, and $d$ is the length of the cavity, $330 \mu \mathrm{m}$ for the sample in Fig. 9. By considering the peaks at 1546.4 and $1550 \mathrm{~nm}$, a FSR of $\sim 3.6 \mathrm{~nm}$ is calculated. The average peak separation between all observable peaks is $\sim 3.8 \mathrm{~nm}$.

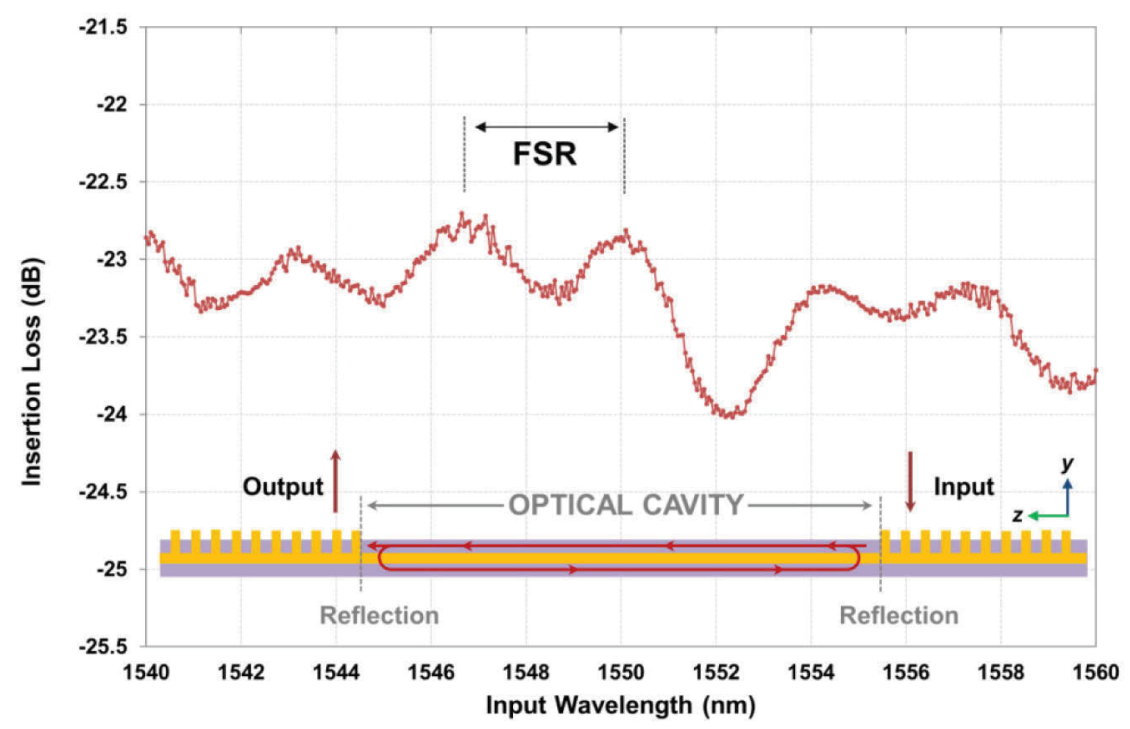

Fig. 9. Measured insertion loss as a function of optical wavelength for a $\mathrm{Au}$ only waveguide section. Diagram of the optical cavity formed by the gratings shown in inset.

The finesse of the cavity is described by the equation

$$
F=F S R / F W H M
$$

where $F W H M$ is the full-width at half-maximum of the peaks. For the peak at $1550 \mathrm{~nm}$, the finesse is 2.1 which equates to a grating reflectance of 0.28 . This compares well with the simulated finesse of 2.0 which would have a reflectance of 0.27 . Wavelength resonance is unavoidable in a grating-coupled design however it is not particularly problematic.

\section{Concluding remarks}

LRSPP cladded membrane waveguides with grating couplers have been demonstrated for the first time. The waveguides have been characterized with a cutback technique, with the $\mathrm{Au}$ waveguides having a measured MPA of $3.4 \mathrm{~dB} / \mathrm{mm}$, while the $\mathrm{Pd} / \mathrm{Au}$ waveguides having a measured MPA of $57 \mathrm{~dB} / \mathrm{mm}$. The total coupling losses from the gratings was measured to be approximately $18.4 \mathrm{~dB}$. The performance is quite sensitive to the structural dimensions as fabrication errors resulted in deviations from the simulated behavior. The grating couplers induce a mild Fabry-Pérot resonance. 


\section{Chapter: Hydrogen Sensing}

The following manuscript entitled "Hydrogen sensing with $\mathrm{Pd}$-coated long-range surface plasmon membrane waveguides" has been submitted to the journal Nanoscale and is currently under review.

The article represents work performed solely by the primary author under the supervision and guidance of the co-authors. It details the methodology and results of hydrogen sensing characterization of the cladded membrane waveguide sensor. The experiments were performed at the Berini Lab at the University of Ottawa. 


\section{Nanoscale}

\section{ARTICLE}

\section{Hydrogen sensing with Pd-coated long-range surface plasmon membrane waveguides}

Received 00th January 20xx, Accepted 00th January 20xx

DOI: $10.1039 / x 0 x \times 00000 x$

www.rsc.org/

\author{
Norman R. Fong, ${ }^{a^{*}}$ Pierre Berini ${ }^{b, c}$ and R. N. Tait ${ }^{a}$
}

A low power, reusable optical hydrogen sensor using long-range surface plasmon polariton (LRSPP) cladded membrane waveguides is demonstrated. The sensor incorporates a $5 \mu \mathrm{m}$ wide, $20 \mathrm{~nm}$ thick gold stripe embedded in a $160 \mathrm{~nm}$ thick free-standing Cytop membrane with a $5 \mathrm{~nm}$ thick Pd over-layer. Input and output coupling is achieved with directly integrated broadside grating couplers. The sensor is tested with hydrogen concentrations up to $3 \%$ and demonstrates a strong response with an estimated detection limit of $290 \mathrm{ppm}$, and a response time of $7 \mathrm{~s}$ to a $0.6 \% \mathrm{H}_{2}$ step - this level of performance is among the best reported for optical $\mathrm{H}_{2}$ sensors. Cycling of the hydrogen exposure shows a significant hysteresis response, however no film deformation or delamination was observed over many cycles. The high stress that is induced in clamped films during hydrogenation is relaxed in due to the film being deposited on the flexible and elastic Cytop membrane. This could result in improved lifetimes for this sensor and increased uptake ability.

\section{Introduction}

Hydrogen gas $\left(\mathrm{H}_{2}\right)$ is flammable at concentrations ranging from $4 \%-75 \%$ in air, with a low ignition energy and high heat of combustion. There has been an increase in its industrial application as a clean energy source ${ }^{1,2}$ which has precipitated the need for safer, more reliable and increasingly compact sensors than currently available. Sensors commonly use palladium ( $\mathrm{Pd}$ ) as a transducer as it absorbs hydrogen proportional to the atmospheric concentration forming palladium hydride $\left(\mathrm{PdH}_{\mathrm{x}} \text { where } \mathrm{x} \text { is the atomic ratio of } \mathrm{H}: \mathrm{Pd}\right)^{3}$.

$\mathrm{H}_{2}$ sensors have been developed based on catalytic reactions ${ }^{4}$, thermoelectric responses ${ }^{5}$, work-function response ${ }^{6}$, electrochemical effects ${ }^{7}$, resistance changes ${ }^{8,9}$ and chemo-mechanical responses ${ }^{10}$. Catalytic sensors are common, but require oxygen to be present in order to operate, and also produce a non-selective response to other combustible hydrocarbons ${ }^{11}$. Sensors based on electrical effects typically require resistive/heating elements which can pose a risk of ignition. These methods are also highly temperature sensitive. Optical sensors are less intrusive than their electrical counterparts because the interrogating optoelectronics (and electronics) can be located remotely from the sensing element and sensing environment using low-loss optical fibres. This also completely eliminates any ignition risk and renders the sensors immune to EM interference. Optical sensors detect shifts in the optical properties of $\mathrm{PdH}_{\mathrm{x}}$ and do

\footnotetext{
a. Department of Electronics, Carleton University, Ottawa, Ontario, K1S 5B6, Canada ${ }^{b}$. School of Electrical Engineering and Computer Science, Department of Physics, University of Ottawa, Ottawa, Ontario, K1N 6N5, Canada

c. Centre for Research in Photonics, University of Ottawa, Ottawa, Ontario, K1N 6 N5, Canada

*nofong@doe.carleton.ca
}

not require oxygen to facilitate a surface reaction thus making them the safest alternative. Sensors based on optical fibres ${ }^{12}$, fibre Bragg gratings ${ }^{13,14}$, SOI waveguides $^{15,16}$, plasmonic nanostructures ${ }^{17,18}$ and hybrid-plasmon waveguides ${ }^{19}$ have been previously examined.

A subset of optical sensors that is of particular interest is plasmonic sensors and specifically surface plasmon polariton (SPP) sensors. SPPs are TM-polarized electromagnetic waves that propagate along the interface between a metal and a dielectric. They have high surface confinement which has encouraged their use as very sensitive biosensors ${ }^{20}$. Hydrogen sensors based on SPP waveguides ${ }^{21-23}$ and nanowires ${ }^{24,25}$ have been reported with high sensitivity and low detection limits. The primary drawback of SPPs is the large optical losses which limit the sensor size and applications. The long-range surface plasmon polariton (LRSPP) is a low-loss (and less-confined) SPP, that propagates along a thin slab or stripe, symmetrically cladded in dielectric. LRSPPs have been successfully demonstrated for hydrogen sensing using a modified Kretschmann configuration with a Pd film on a 1-D photonic crystal $^{26,27}$, however this setup can be bulky due to the prism coupler. An alternative is to use an integrated optical planar LRSPP stripe waveguide which has been shown to be very competitive for biosensing applications because a long optical interaction length can be engineered within the sensing region ${ }^{28}$. These structures can be excited in an end-fire manner or via grating couplers and result in more compact implementations compared to prism-coupled LRSPPs. Additionally, planar devices can be mass manufactured on wafers to lower the unit cost compared to Krestschmann structures and fibre sensors. This article presents the first experimental realization of an integrated optical LRSPP cladded membrane waveguide ${ }^{29}$ as a hydrogen sensor. The 
sensor features a Pd transducer which produces a change in the measured output optical power upon hydrogen absorption. The sensor also uses directly-integrated grating couplers for input and output coupling to cleaved optical fibres.

\section{Materials and Methods}

\subsection{Palladium-Hydrogen Interactions}

Pd quickly and reversibly absorbs hydrogen, with solubility dependent on the partial pressure and temperature of exposure. Hydrogen gas initially adsorbs onto the surface of $\mathrm{Pd}$ which disassociates $\mathrm{H}_{2}$ molecules, allowing $\mathrm{H}$ to diffuse into the bulk forming $\mathrm{PdH}_{\mathrm{x}}$ until equilibrium is established. The dynamics of $\mathrm{Pd}$ and $\mathrm{H}$ interaction has been investigated thoroughly ${ }^{30,31} \cdot \mathrm{PdH}_{\mathrm{x}}$ exists in two solid phases depending on the level of hydrogen content, as well as the temperature and pressure, referred to as the $\alpha$ - and $\beta$-hydride phases. The $\alpha$ phase occurs at low concentrations where disassociated hydrogen atoms will randomly situate themselves at interstitial locations within the Pd lattice. At higher concentrations the interaction between hydrogen atoms will result in a more ordered positioning resulting in added lattice strain, producing the $\beta$-phase and causing a large shift in the lattice constant ${ }^{32}$. At temperatures below $\sim 310{ }^{\circ} \mathrm{C}$ the metal can exist as a mixture of both phases $(\alpha+\beta)$. It has been observed that the transition to this $\alpha+\beta$ phase induces significant hysteresis in the pressure-composition relation ${ }^{33}$, while no hysteresis exists in the pure $\alpha$ phase. It has also been observed that thin $\mathrm{Pd}$ films $(<6 \mathrm{~nm})$ have significantly reduced uptake capability which causes them to saturate at lower concentrations ${ }^{34,35}$.

The permittivity (complex dielectric function) of metals can be represented using models such as the Drude-Lorentz model or the Brendel-Bormann model ${ }^{36}$. At optical and infrared wavelengths the permittivity of $\mathrm{Pd}$ is that of a lossy dielectric. In Pd, hydrogen uptake changes the electron density and electron collision frequency, and causes a physical expansion of the metal lattice, which produces a multitude of effects on the optical properties of the metal ${ }^{37,38}$. The combined effect of these changes can be modelled crudely as a net scaling of the permittivity of the palladium hydride following a simple scaling equation $^{39}$

$$
\varepsilon_{P d H x}(c)=h(c) \cdot \varepsilon_{P d}
$$

where $c$ is the hydrogen concentration and $h(c)$ is the scaling factor.

\subsection{Sensing platform}

The LRSPP cladded membrane waveguide hydrogen sensor is diagrammed in Fig. 1a-b. It consists of a $5 \mu \mathrm{m}$ wide and $20 \mathrm{~nm}$ thick Au stripe symmetrically embedded in a $160 \mathrm{~nm}$ thick free-standing Cytop membrane in air. A section of the waveguide has an overlaid $5 \mathrm{~nm}$ thick Pd patch. Fig. 1c-d show LRSPP mode profiles ( $y$-component of electric field) in the two regions computed using the finite-element method (FEM). Input and output optical coupling from cleaved optical fibres is

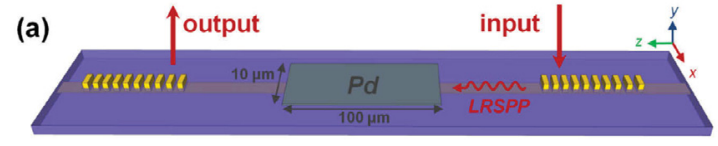

(b)

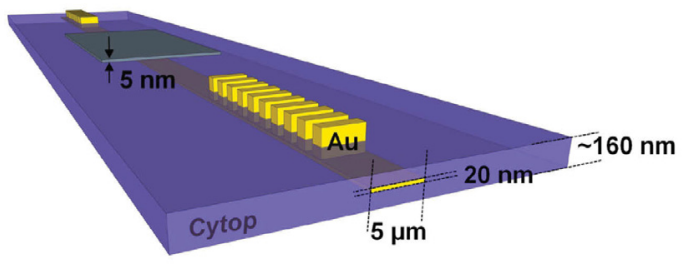

(c)

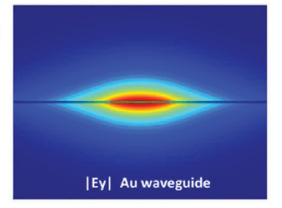

(d)

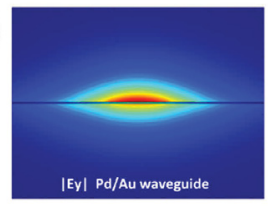

Fig. 1. (a)-(b) LRSPP cladded membrane waveguide $\mathrm{H}_{2}$ sensor with broadside grating couplers. Dimensions labelled but not depicted to scale. For simplicity, the substrate is omitted from view. (c) Mode profile in the Au waveguide section. (d) Mode profile in the $\mathrm{Pd} / \mathrm{Au}$ waveguide section.

achieved via directly integrated grating couplers. Broadside (perpendicular) coupling is preferred for this type of structure as end-fire coupling would require a precisely cleaved or diced end facet which cannot be easily achieved for a free-standing membrane without compromising the structure. The grating bumps are $225 \mathrm{~nm}$ thick, $740 \mathrm{~nm}$ wide with a $1380 \mathrm{~nm}$ period $^{29}$. The sensor was designed to be as compact as possible with $\mathrm{Pd}$ patches between $90 \mu \mathrm{m}$ and $190 \mu \mathrm{m}$ in length. The total separation between gratings is $\sim 300 \mu \mathrm{m}$ which allows for clearance for two perpendicularly-aligned cleaved optical fibres without additional focusing.

The membrane material is Cytop, which is a low-refractive index ( 1.34) soft and flexible amorphous fluoropolymer, previously demonstrated in other LRSPP devices ${ }^{40-45}$. The sensors are fabricated on 2 " silicon wafers which are patterned and wet etched to form the membrane ${ }^{46}$. For simplicity, the silicon substrate is hidden from view in Fig. 1 . The waveguide design $^{29}$ and passive optical characterization ${ }^{47}$ have been previously explored.

\subsection{Interrogation Setup}

The device is excited by a single-mode polarizationmaintaining (PM) optical fibre perpendicularly-aligned to the input grating and a diode laser source emitting at $1550 \mathrm{~nm}$. The fibre is mounted on a curved aluminium post, fitted to a six-axis micro positioner as diagrammed in Fig. 2a. An output multimode (MM) fibre is mounted on an oppositely placed curved post, fitted to another positioner, and connected to an optical power meter (EXFO-1600). The fibres are positioned under microscope with the input polarization direction aligned 

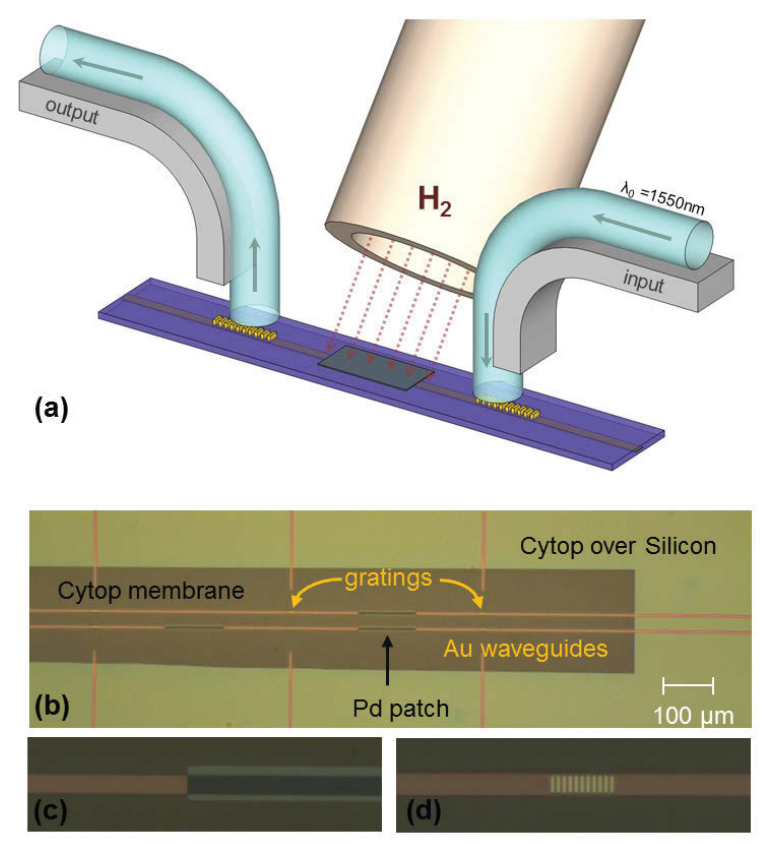

Fig. 2. (a) Diagram of fibre alignment and gas exposure set-up over a sensor. (b) Microscope image of a $\mathrm{H}_{2}$ sensor. (c) High-magnification microscope image of a Pd patch. (d) High-magnification microscope image of a grating.

along the direction of the waveguide, perpendicular to the grating. The source gas is a mixture of $3 \% \mathrm{H}_{2}$ in nitrogen $\left(\mathrm{N}_{2}\right)$. A pure $\mathrm{N}_{2}$ source is also used to dilute the concentration to intermediate values via a mass flow control system. The gas flow feeds a syringe needle tube that is mounted on a positioner and placed $\sim 2 \mathrm{~mm}$ from the sensor. Fig. $2 \mathrm{~b}$ shows a microscope image of a fabricated sensor which has two parallel waveguides and three $\mathrm{Pd}$ patches on a single membrane. Fig. $2 c-d$ show magnified views of a Pd overlay and a grating respectively. The apparatus is assembled on an optical table and enclosed with opaque walls to block external light and variations in ambient air flow.

The inner tube diameter is $\sim 500 \mu \mathrm{m}$ which is significantly larger than the size of the sensor, therefore a hermetic flow cell is not necessary as low to moderate flow rates will produce consistent and complete exposure of the sensor. This direct exposure method minimizes the exchange time (i.e., the time it takes for the gas delivery to reach the active element of the sensor). Although this setup is simple, it has some drawbacks as the gas flow will displace the optical fibres, which for experimental purposes are not permanently secured but rather temporarily held in place. This results in a flow-rate dependent signal shift caused by misalignment. For testing purposes, this can be managed by keeping the flow rate constant when cycling between hydrogen and nitrogen. Ideally, the fibres would be permanently mounted which would eliminate this issue.

\subsection{Stability and Noise}

The passive (no $\mathrm{H}_{2}$ ) insertion loss of the sensor is observed over an extended period (>60 min) such that the ambient noise level and stability can be determined. The stability of the signal refers to long term drift in the measured output power at a constant input. The laser diode and controller should provide a stable low-noise signal. Coupling is polarization dependent, therefore mechanical stability at the input fibre is critical. The input fibre micropositioner provides good experimental control by providing multiple degrees of freedom; however this results in lower stability and increased noise due to the larger number of mechanical components. In addition, fabrication defects (particulates, metal roughness) can result in additional noise due to optical scattering within the device, and this can vary between devices.

\section{Experimental Results and Discussion}

\subsection{Hydrogen Response}

A $100 \% \mathrm{~N}_{2}$ flow rate of $500 \mathrm{sccm}$ is used to establish a baseline signal at room temperature. The gas was switched to $3 \% \mathrm{H}_{2}$ (balance $\mathrm{N}_{2}$ ) at the same flow rate and allowed to stabilize. The gases were cycled, as shown in Fig. 3, while measuring output power $(\mathrm{dBm})$ against time. The input laser is set to deliver $0 \mathrm{dBm}$ with the majority of the loss in the system coming from grating coupling and misalignments ${ }^{47}$.

Hydrogenation of a Pd film adds to the number of free electrons in the metal ${ }^{48}$ as well as increasing the scattering centres which increase the collision frequency. These effects collectively would result in an increase in the plasma frequency which should cause an increase in attenuation. However, the opposite response is observed in Fig. 3 where a decrease in attenuation (increased output power) occurs. It is believed that thicker Pd films are dominated by lattice volume expansion which result in a decrease in the electron density

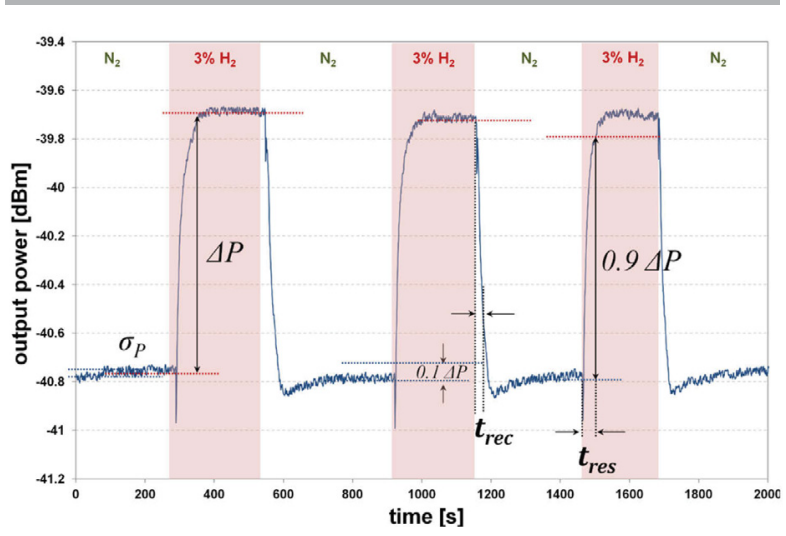

Fig. 3. Sensor response due to exposure to $3 \% \mathrm{H}_{2}$ in $97 \% \mathrm{~N}_{2}$, cycled with $100 \% \mathrm{~N}_{2}$. The parameters of interest are the noise level $\left(\sigma_{P}\right)$, signal step $(\Delta P)$, response time $\left(t_{\text {res }}\right)$ and recovery time $\left(t_{\text {rec }}\right)$ as defined on the figure. 

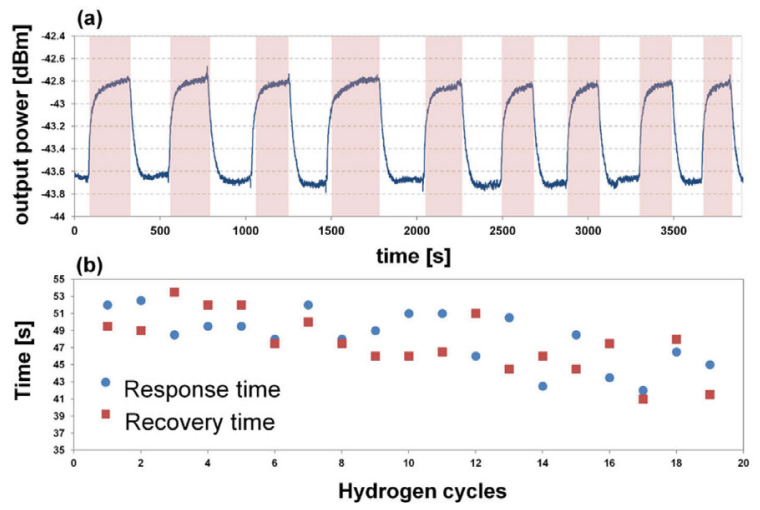

Fig. 4. (a) Several cycles of $3 \%$ hydrogen exposure with minimal variation in step time or noise level. (b) Sensor response time and recovery time over various cycles

and thus cause a decrease in attenuation ${ }^{49}$. This behaviour is consistent with other sensors of similar film thickness ${ }^{16,39,50,51 \text {, }}$.

The noise levels $\left(\sigma_{P}\right)$ are calculated as the standard deviation of the steady state signals (in $\mathrm{nW}$ ) and have a value of $\sim 0.25 \mathrm{nW}$. This is an insignificant variation from the ambient state (no gas flow) which confirms that the gas flow does not substantially impact the noise. The sensor signal $(\Delta P)$ is computed as step difference between the averaged steady state signals (in $\mathrm{nW}$ ) and is approximately $\sim 23.5 \mathrm{nW}$ resulting in a signal-to-noise ratio $\left(\Delta P / \sigma_{P}\right.$ or SNR) of $\sim 94$ for these three cycles. The process was repeated over 20 cycles with an insignificant variation in the SNR over time. Returning to the numerical modelling of the sensor ${ }^{29}$, this observed decrease in corresponds to a $\sim 10 \%$ reduction in the permittivity such that $h(c) \sim 0.9$.

\subsection{Response and Recovery Time}

Another important figure of merit for hydrogen sensors is the response time and the recovery time. The response time $\left(t_{\text {res }}\right.$ or $\left.t_{90}\right)$, is defined as the time to reach $90 \%$ of the steady state signal level. Similarly, the recovery time $\left(t_{\text {rec }}\right.$ or $\left.t_{10}\right)$ is the fall time to $10 \%$ of the original signal. Fig. 4 a shows a sample output over several $3 \%$ hydrogen cycles. Fig. $4 \mathrm{~b}$ plots the response and recovery times for 19 cycles of exposure. There is a moderate variation from cycle-to-cycle which can most likely be attributed to minor fluctuations in the noise level or stability over time. The response and recovery times are similar and show a slight decrease from about $50 \mathrm{~s}$ to $45 \mathrm{~s}$ with increasing cycles.

Most optical sensors based on thin planar Pd films have shown similar response times when exposed to comparable concentrations of $\mathrm{H}_{2}(\sim 3 \%)$. The response time is limited primarily by three factors: exposure concentration, surface area and thickness. The sensor response stabilizes when the palladium hydride reaches equilibrium with the environment, which will occur more quickly for lower concentrations. Fig. 5 shows that the response time is reduced to $7 \mathrm{~s}$ for a $0.6 \%$ exposure. Larger film surface areas also improve the response.

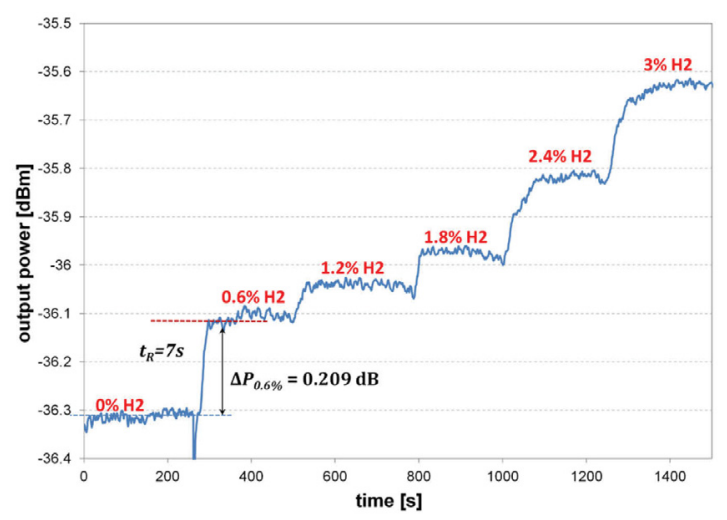

Fig. 5. Sensor response to $0.6 \%$ hydrogen intervals showing a non-linear sensitivity.

Thinner films will saturate more quickly which can also improve response times.

\subsection{Detection Limit}

The $\alpha-\beta$ solid phase change of $\mathrm{PdH}_{\mathrm{x}}$ results in a non-linear relation between the permittivity and concentration. Fig. 5 shows the output response in the concentration range of 0 $3 \%$ in steps of $0.6 \%$. The sensor was operated at room temperature for which the transition into the mixed $(\alpha-\beta)$ phase occurs at around $2 \% \mathrm{H}_{2}$. The first step (from 0 to $0.6 \%$ ) is noticeably larger than the subsequent steps within the $\alpha$ phase, likely due to initial nucleation of $\mathrm{H}$ atoms at the surface which would result in a larger initial response. The rate of nucleation has likely stabilized in subsequent steps which are responding mostly due to changes in the permittivity of the film. The transition into the mixed $(\alpha-\beta)$ phase is evident in the measured response near $\sim 1000 \mathrm{~s}$ where a large step in signal is observed.

The lowest controllable concentration in our set-up was $0.6 \%$; however, it is clear from Fig. 5 that the minimum detectable concentration is much lower. The theoretical detection limit is defined as the concentration resulting in a SNR of 1 . Although the initial response within the $\alpha$ region is only semi-linear, it can be approximated as linear for the purposes of estimating the detection limit, yielding an estimated value of $0.029 \%$ or $290 \mathrm{ppm}$, which is comparable to or better than similar structures ${ }^{16,22,52}$.

\subsection{Hysteresis}

Pd films are well-known to show hysteresis from cycled hydrogen exposure ${ }^{53-55}$. When a Pd film has transitioned into the $\beta$-hydride phase, the more ordered distribution of $\mathrm{H}$ atoms in the lattice results in an increased activation energy required for desorption and return to the $\alpha$ phase $^{53}$, resulting in lowered desorption response and hence signal hysteresis. Bulk $\mathrm{Pd}$ films deposited on elastically rigid substrates experience high compressive stresses ${ }^{56}$ as they restrict in-plane expansion. Thus the large lattice expansion experienced with hydrogen 
uptake can lead to film cracking and delamination. Hysteresis and deformation can be minimized by delaying or eliminating transition to the $\beta$-hydride phase. Many Pd-based sensors address this issue by using very thin films $(<5 \mathrm{~nm})$. These films experience increased tensile stiffness due to clamping to a rigid substrate, effectively restricting hydrogen uptake and thus limiting lattice expansion. Alternatively, a Pd alloy (e.g. Palladium-nickel) can be used instead of pure Pd to reduce the $\mathrm{H}_{2}$ uptake and associated strain ${ }^{57-59}$. These techniques will, however, limit the dynamic range of the sensor by restricting the maximum $\mathrm{H}_{2}$ capacity of the film, which is not necessarily desirable.

Fig. 6 shows a hysteresis plot of the cycled hydrogen response $(0.6 \%$ steps). At the highest concentration tested, the signal has not yet saturated. Despite the observed hysteresis, no cracking or deformation of the film was observed during the experiments in which devices were cycled up to 30 times. The LRSPP waveguide is highly sensitive to the roughness of the film surfaces such that any cracking or delamination would result in a significant noise increase over successive cycles, which was not observed.

It has been reported that $\mathrm{Pd}$ clusters embedded in elastically soft polymers have enhanced $\mathrm{H}$-solubility from reduced interfacial stress ${ }^{60}$. In the case of the membrane waveguide sensor, the Pd film is deposited on top of a soft and elastic Cytop membrane which should reduce substrate clamping and compressive stress during hydrogen absorption, leading to enhanced $\mathrm{H}$-solubility and reusability.

\subsection{Performance Improvements}

The U.S. Department of Energy requirements ${ }^{61}$ for $\mathrm{H}_{2}$ sensors states that the target standard for sensor response time should be $<1 \mathrm{~s}$ within the dynamic range of $0.1 \%-10 \%$ operating at temperatures between $-30^{\circ} \mathrm{C}$ to $80^{\circ} \mathrm{C}$. To date, no sensor has been demonstrated to fully meet these strict requirements, most often only satisfying one parameter among detection limit, sensitivity, dynamic range or response time.

Our estimated detection limit of $0.029 \%$ ppm exceeds the target limit and is comparable to other optical sensors in the literature ${ }^{16,22,52,62,63}$. The detection limit can be improved by reducing the noise floor and/or increasing the signal step. A significant amount of noise is inherent in the experimental setup from the number of non-fixed mechanical axes, which are only present for experimental purposes. The signal step size is proportional to the length of the Pd covered section of the waveguide. The sensor was designed to be compact and thus the length of the Pd patch was limited to no more than $190 \mu \mathrm{m}$. However the waveguide only accounts for approximately $10 \mathrm{~dB}$ loss in the system with the majority of the power loss coming from the grating couplers. Thus the Pd patch size could easily be increased, increasing the SNR while still maintaining an acceptable signal level.

The full dynamic range of our sensor was not examined as very few sensors are tested above $4 \% \mathrm{H}_{2}$ for safety reasons. It is thought that the sensor should have increased hydrogen

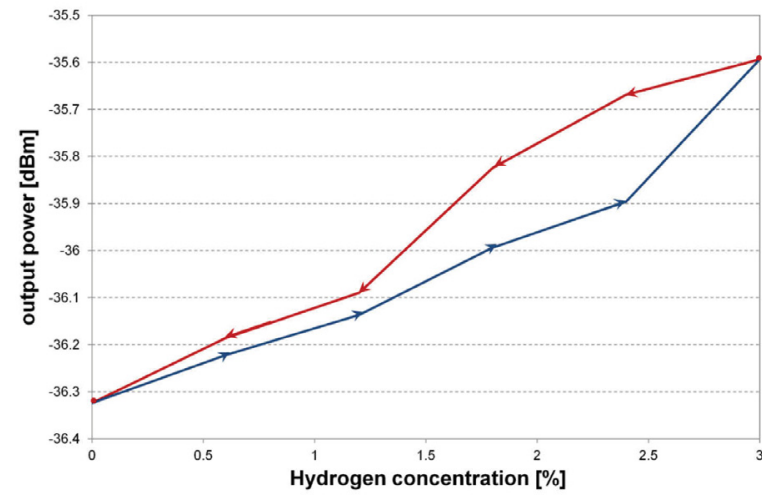

Fig. 6. Sensor response to $0.6 \%$ hydrogen intervals showing a non-linear sensitivity.

storage capability compared to other sensors of equal Pd film thickness, due to the reduced interfacial stresses of the Pd film deposited on the elastically soft Cytop membrane.

The experiments presented were performed at room temperature, so further characterization is required as the pressure-concentration-temperature isotherms of palladium hydride $^{3}$ reveal temperature dependence. Transition to the mixed $(\alpha+\beta)$ hydride phase is delayed with increased temperature which has a significant effect on the response of any Pd-based sensor. Additionally, Cytop has a thermal expansion coefficient ${ }^{64}$ of $7.4 \times 10^{-5} \mathrm{~K}^{-1}$ which is higher than that of $\mathrm{Au}\left(14 \times 10^{-6}\right)$ and could cause a change in the grating pitch with temperature which will affect the coupling efficiency. However, the couplers are broadband and any effect could likely be compensated by referencing.

The most difficult requirement to meet is the sub $1 \mathrm{~s}$ response time. Although this sensor demonstrated a response time as low as $7 \mathrm{~s}$ to $0.6 \%$ exposure, the time increases to $\sim 50$ $s$ at $3 \%$. Reducing the film thickness can improve response time but will further limit the storage capacity. The response time could be improved by nanostructuring the Pd layer such as implementing nanogaps ${ }^{65}$. Delaying hydride phase transition by using a $\mathrm{Pd}$ alloy has also been shown to improve response time ${ }^{51}$ however this also reduces sensitivity and capacity.

\section{Conclusion}

A low-power, compact hydrogen sensor using a LRSPP cladded membrane waveguide is demonstrated at $\mathrm{H}_{2}$ concentrations up to $3 \%$ with an estimated detection limit of $290 \mathrm{ppm}$ and a response time of $7 \mathrm{~s}$ to $0.6 \% \mathrm{H}_{2}$. The detection limit is comparable to other optical sensors and could be improved by increasing the length of the Pd patch. The sensors show hysteresis upon hydrogen cycling without any observable deformation of the Pd film. This is due to the reduced stress in the Pd film because it rests on a soft and elastic Cytop membrane, which should lead to a larger dynamic range compared to films on rigid structures while also having improved lifetime and reusability. 


\section{References}

1 A. Kirubakaran, S. Jain and R. K. Nema, Renewable Sustainable Energy Rev., 2009, 13, 2430-2440.

2 S. Hardman, R. Steinberger-Wilckens and D. van der Horst, Int. J. Hydrogen Energ., 2013, 38, 15438-15451.

3 F. A. Lewis, Platinum Metals Rev. 1960, 4, 132-137

4 E. Lee, I. Hwang, J. Cha, H. Lee, W. Lee, J. J. Pak, J. Lee and B. Ju, Sens. Actuators, B, 2011, 153, 392-397.

5 W. Shin, K. Tajima, Y. Choi, N. Izu, I. Matsubara and N. Murayama, Sens. Actuators, B, 2005, 108, 455-460.

6 A. Trinchi, S. Kandasamy and W. Wlodarski, Sens. Actuators, B, 2008, 133, 705-716.

7 G. Korotcenkov, S. D. Han and J. R. Stetter, Chem. Rev., 2009, 109, 1402-1433.

8 J. Hong, S. Lee, J. Seo, S. Pyo, J. Kim and T. Lee, ACS Appl. Mater. Interfaces, 2015, 7, 3554-3561.

9 Z. Wang, Z. Li, T. Jiang, X. Xu and Wang, ACS Appl. Mater. Interfaces, 2013, 5, 2013-2021.

10 J. Henriksson, L. G. Villanueva and J. Brugger, Nanoscale, 2012, 4, 5059-5064.

11 T. Hübert, L. Boon-Brett, G. Black and U. Banach, Sens. Actuators, B, 2011, 157, 329-352.

12 M. Yang and J. Dai, Photonic Sens., 2014, 4, 300-324.

13 K. Schroeder, W. Ecke and R. Willsch, Opt. Laser Eng. 2009, 47, 1018-1022.

14 J. Dai, M.Yang, X. Yu and H. Lu, Opt. Fiber Technol, 2013, 19, 26-30.

15 M. Z. Alam, N. Carriere, F. Bahrami, M. Mojahedi and J. S. Aitchison, Opt. Lett. 2013, 38, 1428-1430.

16 N. Carriere, M. Z. Alam, M. Mojahedi and J. S. Aitchison, Sens. Actuators, B, 2015, 216, 6-10.

17 C. Langhammer, I. Zorić, B. Kasemo and B. M. Clemens, Nano Lett., 2007, 7, 3122-3127.

18 C. Wadell and C. Langhammer, Nanoscale, 2015, 7, 1096310969.

19 F. Gu, G. Wu and H. Zeng, Nanoscale, 2015, 7, 924-929.

20 S. A. Maier and H. A. Atwater, J. App. Phys., 2005, 98, 011101.

21 B. Chadwick, J. Tann, M. Brungs and M. Gal, Sens. Actuators, B, 1994, 17, 215-220.

22 C. Perrotton, R. Westerwaal, N. Javahiraly, M. Slaman, H. Schreuders, B. Dam and P. Meyrueis, Opt. Express, 2013, 21, 382-390.

23 N. R. Fong, P. Berini and R. N. Tait, Sens. Actuators, B, 2012, 161, 285-291.

24 F. Gu, H. Zeng, L. Tong and S. Zhuang, Opt. Lett., 2013, 11, 1826-1828.

25 F. Gu, H. Zeng, Y. B. Zhu, Q. Yang, L. K. Ang and S. Zhuang, Adv. Opt. Mater., 2014, 2, 189-196.

26 V. N. Konopsky, D. V. Basmanov, E. V. Alieva, D. I. Dolgy, E. D. Olshansky, S. K. Sekatskii and G. Dietler, New J. Phys., 2009, 11, 063049

27 V. N. Konopsky and E. V. Alieva, Opt. Express, 2009, 4, 479481.

28 P. Berini, Adv. Opt. Photonics, 2009, 1, 484-588.

29 N. R. Fong, P. Berini and N. Tait, J. App. Phys., 2015, 117, 163108.

30 H. Brüning and A. Sieverts, Z. Phys. Chem. 1933, 163, 409.

31 R. J. Wolf, M. W. Lee, R. C. Davis, P. J. Fay and J. R. Ray, Phys. Rev. B: Condens. Matter Mater. Phys., 1993, 48, 12415.

32 R. J. Maier, B. J. S. Jones, J. S. Barton, S McCulloch and T. Allsop, J. Opt. A: Pure Appl. Opt. 2007, 9, S45-S59.

33 D. H. Everett and P. Nordon, P. Roy. Soc. Lond. A. Mat.,1960, 259, 341-360.

34 J. A. Garcia and A. Mandelis, Rev. Sci. Instrum., 1996, 67, 3981-3983.
35 G. A. Frazier and R. Glosser, J. Less-Common Met.,1980, 74, 89-96.

36 A. D. Rakić, A. B. Djurišić, J. M. Elazar and M. L. Majewski, Appl. Opt. 1998, 37, 5271-5283.

37 W. E. Vargas, I. Rojas, D. E. Azofeifa and N. Clark, Thin Solid Films, 2006, 496, 189-196.

38 W. E. Vargas, D. E. Azofeifa, N. Clark, H. Solis and F. Montealegre, Appl. Opt. 2014, 53, 5294-5306.

39 X. Bévenot, A. Trouillet, C. Veillas and H. Gagnaire, Meas. Sci. Technol., 2001, 13, 118-124.

40 N. R. Fong, P. Berini and R. N. Tait, J. Microelectromech. Syst., 2010, 19, 700-705.

41 H. Fan and P. Berini, Appl. Opt., 2013, 54, 162-170.

42 H. Fan, R. Buckley and P. Berini, Appl. Opt., 2012, 51, 14591467.

43 O. Krupin, H. Asiri, C. Wang, R. N. Tait and P. Berini, Opt. Express, 2013, 21, 698-709.

44 B. Banan, M. S. Hai, E. Lisicka-Skrzek, P. Berini and O. Liboiron-Ladouceur, IEEE Photonics J., 2013, 5, 2201811.

45 P. Béland, O. Krupin and P. Berini, Biomed. Opt. Express, 2015, 6, 2908-2922.

46 N. R. Fong, P. Berini and N. Tait, J. Vac. Sci. Technol. A, 2015, 33, 021201.

47 N. R. Fong, P. Berini and R. N. Tait, Opt. Express, 2015, 23, 17421-17430.

48 F. A. Lewis, Platinum Met. Rev. 1971, 15, 21.

49 V. N. Konopsky, D. V. Basmanov, E. V. Alieva, S. K. Sekatskii, and G. Dietler, Appl. Phys. Lett. 2012, 100, 083108.

50 D. Zalvidea, A.Diez, J.L. Cruz and M.V. Andres, Sens. Actuators, B, 2005, 114, 268-274.

51 D. Monzón-Hernández, D. Luna-Moreno and D. MartínezEscobar, Sens. Actuators, B, 2009, 136, 562-566.

52 J. Dai, M. Yang, Z. Yang, Z. Li, Y. Wang, G. Wang, Y. Zhang, Z. Zhuang, Sens. Actuators, B, 2014, 190, 657-663.

53 E. Lee, J. M. Lee, J. H. Koo, W. Lee and T. Lee, Int. J. Hydrogen Energ., 2010, 35, 6984-6991.

54 N. A. Scholtus and W. K. Hall, J. Chem. Phys., 1963, 39, 868.

55 J. C. Barton, F. A. Lewis and I. Woodward, Trans. Faraday Soc., 1963, 59, 1201-1207.

56 T. P. L. Pedersen, C. Liesch, C. Salinga, T. Eleftheriadis, H. Weis and M. Wutting, Thin Solid Films, 2014, 458, 299-303.

57 R. C. Hughes and W. K. Schubert, J. Appl. Phys., 1992, 71, 542.

58 Y. Cheng, Y. Li, D. Lisi and M. W. Wang, Sens. Actuators, B, 1996, 30, 11-16.

59 E. Lee, J. M. Lee, E. Lee, J. Noh, J. H. Joe, B. Jung and W. Lee, Thin Solid Films 2010, 519, 880-884.

60 A. Pundt, C. Sachs, M. Winter, M. T. Reetz, D. Fritsch and R. Kirchheim, J. Alloys Compd. 1999, 293-295, 480-483.

61 W. J. Buttner, M. B. Post, R. Burgess and C. Rivkin, Int. J. Hydrogen Energ., 2011, 36, 2462-2470.

62 J. Villatoro and D. Monzón-Hernández, Opt. Express, 2005, 13, 5087-5092.

63 A. Tittl, P. Mai, R. Taubert, D. Dregely, N. Liu and H. Giessen, Nano Lett., 2011, 11, 4366-4369.

64 AGC Chemicals, Cytop Technical Information, http://www.agcce.com/cytop-technical-information/

65 B. Jang, K. Y. Lee, J. Noh and W. Lee, Sens. Actuators, B, 2014, 193, 530-535. 


\section{Chapter: Conclusions and Future Work}

The preceding articles presented the entire evolution of the LRSPP cladded membrane waveguide hydrogen sensor which is the first device of its type to be demonstrated in the literature. The modeling and design of the structure showed that it should function appropriately as both a waveguide and a sensor. The fabrication consisted of a comprehensive process including: blind double-sided alignment, optical and e-beam lithography and through wafer wet etch while adhering to a strict thermal budget.

The waveguides were passively characterized with the $\mathrm{Pd} / \mathrm{Au}$ section having a waveguide loss of $57 \mathrm{~dB} / \mathrm{mm}$ and the Au section having a loss of $3.4 \mathrm{~dB} / \mathrm{mm}$. There is clear potential for improvements as described in Chapter 7 where the fabricated device mildly but noticeably differed in optical performance from the theoretical structure. These results shed light on the fabrication steps which will require additional control to avoid future oversights, specifically noting the critical nature of the structure symmetry.

As a hydrogen sensor, the device has an extrapolated detection limit of $290 \mathrm{ppm}$ which is competitive to other waveguide based sensors. The detection limit could be improved by simply scaling the Pd patch. This would allow for a larger step height due to increased change in total attenuation of the waveguide. This could also add additional noise however the observations during the work done in Chapter 7 showed that the noise levels of longer Pd patches were negligibly different than shorter patches. Thus scaling the Pd patch could allow this device to have the best detection limit among optical techniques. 
The sensor results also reveal that the Cytop elasticity allowed for less restricted hydrogen uptake by the Pd film compared to clamped films on rigid substrates, which can potentially lead to longer lifetimes and possibly a larger detection range. This result was somewhat unexpected prior to experimentation and can lead to further exploration and could potentially lead to other $\mathrm{H}_{2}$ sensors on elastic substrates.

The DOE safety requirements suggest a range of up to $10 \%$, however very few sensors have been demonstrated above $4 \%$, primarily due to the inherent safety concerns of using $\mathrm{H}_{2}$ concentrations above the lower explosive limit within a laboratory atmosphere. Thus further experimentation is required in order to determine the maximum uptake. The requirements also state an operating temperature range of $-30^{\circ} \mathrm{C}$ to $80^{\circ} \mathrm{C}$. All experiments were performed at room temperature. It is not expected that these devices would critically fail at either of these extremes however temperature effects clearly play into the physical characteristics of both the Cytop and the Pd film which requires additional exploration.

Additional experimentation can be done to optimize the grating couplers. Perpendicular excitation was chosen for simplicity but it is the most efficient. Much higher couple efficiency could be achieved with angled excitation. Additionally the grating area could be designed to be wider and taper down to the desired waveguide width which would help with alignment issues.

The technology developed in this thesis is not exclusively purposed for hydrogen sensing. The Pd layer could be replaced with any other functionalization to allow for sensing of other gases such as methane, carbon monoxide, carbon dioxide, etc. The grating couplers did not play a direct role in the actual sensing but are the first of their 
kind to be demonstrated and additionally can be integrated into other LRSPP waveguide type structures where end-fire excitation is impractical or otherwise inconvenient. 


\section{References}

[1] R.W. Wood. "On a remarkable case of uneven distribution of light in a diffraction grating spectrum” Philosophical Magazine Series 64 (21), 396 (1902).

[2] D. Pines. "Collective Energy Losses in Solids" Reviews of Modern Physics 28 (3), 184 (1956).

[3] U. Fano. "Atomic Theory of Electromagnetic Interactions in Dense Materials" Physical Review 103, 1202 (1956).

[4] R. H. Ritchie. "Plasma Losses by Fast Electrons in Thin Films" Physical Review 106, 874 (1957).

[5] National Renewable Energy Laboratory, "Hydrogen \& Fuel Cell Research: Safety, Codes, and Standards", (2015)

http://www.nrel.gov/hydrogen/proj_safety_codes_standards.html

[6] S.A. Maier. "Plasmonics Fundamentals and Applications" Springer US (2007).

[7] W.E. Vargas, "Optical and electrical properties of hydrided palladium thin films studied by an inversion approach from transmittance measurements" Thin Solid Films 496(2), 189 (2006).

[8] A.D. Rakić. "Optical properties of metallic films for vertical-cavity optoelectronic devices" Applied Optics 37(22), 5271 (1998).

[9] P. B. Johnson and R.W. Christy, "Optical Constants of the Noble Metals" Physical Review B 6, 4370 (1972).

[10] H. Raether, "Surface Plasmons on Smooth and Rough Surfaces" Springer Tracts in Modern Physics 111, (1988).

[11] J. Homola. "Electromagnetic Theory of Surface Plasmons", Springer Series on Chemical Sensors and Biosensors 4, 3 (2006).

[12] P. Berini, "Long-range surface plasmon polaritons," Advances in Optics and Photonics 1(3), 484 (2009).

[13] P. Berini and R. Buckley. "On the convergence and accuracy of numerical mode computations of surface plasmon waveguides," Journal of Computational and Theoretical Nanoscience 6, 2040 (2009).

[14] G. Veronis, S. Kocaba, D. A. B. Miller and S. Fan. " "Modeling of Plasmonic Waveguide Components and Networks" Journal of Computational and Theoretical Nanoscience 6, 1808 (2009). 
[15] P. Berini. "Plasmon-polariton waves guided by thin lossy metal films of finite width: Bound modes of asymmetric structures" Physical Review B 63, 125417 , (2001).

[16] T. W. Ebbesen, C. Genet, and S. I. Bozhevolnyi, "Surface plasmon circuitry," Physics Today 61(5), 44 (2008).

[17] P. Berini , R. Charbonneau, and N. Lahoud, "Long-Range Surface Plasmons Along Membrane-Supported Metal Stripes" IEEE Journal of Selected Topics in Quantum Electronics 14(6), 1479 (2008).

[18] M. Tencer and P. Berini "Design of microfluidic channels separated by an ultra-thin free-standing dielectric membrane” Microfluidics and Nanofluidics 6(1), 17 (2008).

[19] P. Berini, N. Lahoud, and R. Charbonneau. "Fabrication of surface plasmon waveguides and integrated components on ultrathin freestanding membranes" Journal of Vacuum Science \& Technology A 26(6), 1383 (2008).

[20] P. Berini, R. Charbonneau, and N. Lahoud. "Long-Range Surface Plasmons on Ultrathin Membranes" Nano Letters 7(5), 1376 (2007).

[21] O. Krupin, H. Asiri, C. Wang, R. N. Tait, and P. Berini. "Biosensing using straight long-range surface plasmon waveguides” Optics Express 21(1), 698 (2013).

[22] X.D. Hoa, A.G. Kirk, and M. Tabrizian. "Towards integrated and sensitive surface plasmon resonance biosensors: A review of recent progress" Biosensors and Bioelectronics 23(2), 151 (2007).

[23] NASA, "Earth Fact Sheet” (2015)

http://nssdc.gsfc.nasa.gov/planetary/factsheet/earthfact.html

[24] F.A. Lewis. “The Palladium Hydrogen System” Academic Press (1967).

[25] T.B. Flanagan and W.A. Oates. "The palladium-hydrogen system" Annual Review of Materials Science 21, 269 (1991).

[26] C. Wadell, S. Syrenova, and C. Langhammer. "Plasmonic Hydrogen Sensing with Nanostructured Metal Hydrides” ACS Nano 8(12), 11925 (2014).

[27] F.D. Manchester. "The H-Pd (Hydrogen-Palladium) System” Journal of Phase Equilibria 15(1), 62 (1994).

[28] R. R. J. Maier, B. J. S. Jones, J. S. Barton, S. McCulloch, T. Allsop, J. D. C. Jones, and I. Bennion. "Fibre optics in palladium-based hydrogen sensing" Journal of Optics A: Pure and Applied Optics 9(6), S45 (2007) 
[29] W. J. Buttner, M. B. Post, R. Burgess, and Carl Rivkin "An overview of hydrogen safety sensors and requirements" International Journal of Hydrogen Energy 36(3), 2462 (2011).

[30] E. Lee, I. Hwang, J. Cha, H. Lee, W. Lee, J. J. Pak, J. Lee, and B. Jua, "Micromachined catalytic combustible hydrogen gas sensor" Sensors and Actuators B: Chemical 153(2), 392 (2011).

[31] T. Hübert, L. Boon-Brett, G. Black, and U. Banach, "Hydrogen sensors - A review" Sensors and Actuators B: Chemical 157(2), 329 (2011).

[32] M. A. Butler, "Fiber Optic Sensor for Hydrogen Concentrations near the Explosive Limit" Journal of the Electrochemical Society 138(9), L46 (1991).

[33] M. Yang, Y. Sun, D. Zhang, and D. Jiang "Using Pd/WO3 composite thin films as sensing materials for optical fiber hydrogen sensors" Sensors and Actuators B: Chemical 143, 750 (2010).

[34] X. Bévenot, A. Trouillet, C. Veillas, H. Gagnaire, and M. Clément, "Hydrogen leak detection using an optical fibre sensor for aerospace applications" Sensors and Actuators B: Chemical 67, 57 (2000).

[35] B. Sutapun, M. Tabib-Azar, and A. Kazemi, "Pd-coated elastooptic fiber optic Bragg grating sensors for multiplexed hydrogen sensing" Sensors and Actuators B: Chemical 60, 27 (1999)

[36] K. Schroeder, W. Ecke, and R. Willsch, "Optical fiber Bragg grating hydrogen sensor based on evanescent-field interaction with palladium thin-film transducer" Optics and Lasers in Engineering 47, 1018 (2009).

[37] G. Ma, C. Li,Y. Luo, R. Mu, and Liang Wang "High sensitive and reliable fiber Bragg grating hydrogen sensor for fault detection of power transformer" Sensors and Actuators B: Chemical 169(5), 195 (2012).

[38] C. Tien, H. Chen, W. Liu, S. Jyu, S. Lin, and Y. Lin "Hydrogen sensor based on side-polished fiber Bragg gratings coated with thin palladium film" Thin Solid Films 516(16), 5360(2006).

[39] D. Luna-Moreno, D. Monzon-Hernandez, J. Villatoro and G. Badences, "Optical fiber hydrogen sensor based on core diameter mismatch and annealed Pd-Au thin films", Sensors and Actuators B: Chemical 125, 66 (2007).

[40] S. Sekimoto, H. Nakagawa, S. Okazaki, K. Fukuda, S. Asakura, T. Shigemori and S. Takahashi, "A fiber-optic evanescent-wave hydrogen gas sensor using palladiumsupported tungsten oxide" Sensors and Actuators B: Chemical 66, 142 (2000). 
[41] J. Villatoro and D. Monzon-Hernandez, "Fast detection of hydrogen with nano fiber tapers coated with ultra thin palladium layers" Optics Express 13, 5087 (2005).

[42] D. Zalvidea, A. Diez, J. L. Cruz and M. V. Andres, "Hydrogen sensor based on palladium-coated fiber-taper with improved time-response" Sensors and Actuators B: Chemical 114, 268 (2006).

[43] A. Ortigosa-Blanch, A. Diez, J. L. Cruz and M. V. Andres, "Time-domain fiber laser hydrogen sensor" Optics Letters 29, 2461 (2004).

[44] M. Z. Alam, N. Carriere, F. Bahrami, M. Mojahedi, and J. S. Aitchison "Pd-based integrated optical hydrogen sensor on a silicon-on-insulator platform" Optics Letters 38(9), 1428 (2013).

[45] C. Langhammer, I. Zorić, B. Kasemo, and B. M. Clemens "Hydrogen Storage in Pd Nanodisks Characterized with a Novel Nanoplasmonic Sensing Scheme" ACS Nano 7(10), 3122 (2007).

[46] B. Chadwick, J. Tann, M. Brungs, and M. Gal "A hydrogen sensor based on the optical generation of surface plasmons in a palladium alloy" Sensors and Actuators B: Chemical 17(3), 215 (1994).

[47] V. N Konopsky, D. V. Basmanov, E. V. Alieva, D. I. Dolgy, E. D. Olshansky, S. K. Sekatskii, and G. Dietler "Registration of long-range surface plasmon resonance by angle-scanning feedback and its implementation for optical hydrogen sensing" New Journal of Physics 11, 063049 (2009).

[48] X. Bévenot, A. Trouillet, C. Veillas, H. Gagnaire, and M. Clément. "Surface plasmon resonance hydrogen sensor using an optical fibre*" Measurement Science and Technology 13(1), 118 (2001).

[49] C. Perrotton, R. J. Westerwaal, N. Javahiraly, M. Slaman, H. Schreuders, B. Dam, and P. Meyrueis . "A reliable, sensitive and fast optical fiber hydrogen sensor based on surface plasmon resonance" Optics Express 21(1), 382 (2013)

[50] P. Tobiška , O. Hugon, A. Trouillet, and H. Gagnaire. "An integrated optic hydrogen sensor based on SPR on palladium" Sensors and Actuators B: Chemical 74(13), 168 (2001)

[51] J. Friend and L. Yeo. "Fabrication of microfluidic devices using polydimethylsiloxane" Biomicrofulidics 4, 026502 (2010).

[52] D. B. Hall, P. Underhill, and J. M. Torkelson. "Spin Coating of Thin and Ultrathin Polymer Film” Polymer Engineering and Science 38(12), 2039 (1998). 
[53] S. Hassan, M. Khodami, R. N. Tait, and P. Berini. "Fabrication of long-range surface plasmon-polariton Bragg gratings with microfluidic channels in Cytop claddings" Microelectronic Engineering 135(5), 38 (2015).

[54] H. Fan, R. Buckley, and P. Beirni. "Passive long-range surface plasmon-polariton devices in Cytop" Applied Optics 51(10), 1459 (2012).

[55] B. Banan, M. S. Hai, E. Lisicka-Skrzek, and P. Berini. "Multichannel Transmission Through a Gold Strip Plasmonic Waveguide Embedded in Cytop" IEEE Photonics Journal 5(3), 2201811 (2013).

[56] AGC Chemicals, “Amorphous Fluoropolymer Cytop” (2015) http://www.bellexinternational.com/products/cytop/

[57] N. R. Fong, P. Berini, and R. N. Tait. "Mechanical Properties of Thin Free-Standing CYTOP Membranes" Journal of Microelectromechanical Systems 19(3), 700 (2010).

[58] N. Fong, P. Berini, and R. N. Tait. "Fabrication of surface plasmon waveguides on thin CYTOP membranes" Journal of Vacuum Science \& Technology A 27, 614 (2009).

[59] R. Daviau, A. Khan, E. Lisicka-Skrzek, R. N. Tait, and P. Berini. "Fabrication of surface plasmon waveguides and integrated components on Cytop" Microelectronic Engineering 87(10), 1914 (2010).

[60] C. Chiu, E. Lisicka-Skrzek, R. N. Tait, and P. Berini. "Fabrication of surface plasmon waveguides and devices in Cytop with integrated microfluidic channels" Journal of Vacuum Science \& Technology B 28, 729 (2010).

[61] M. J. Madou. "Fundamentals of Microfabrication: The Science of Miniaturization" CRC Press, (2002)

[62] O. Tabata, R. Asahi, H. Funabashi, K. Shimaoka, and S. Sugiyama. "Anisotropic etching of silicon in TMAH solutions", Sensors and Actuators A: Physical 34(1), 51 (1992)

[63] E. D. Palik, O. J. Glembocki, I. Heard Jr., P. S. Burno, and L. Tenerz. "Etching roughness for (100) silicon surfaces in aqueous KOH" Journal of Applied Physics 70, 3291 (1991). 